\title{
Search and exploration of hidden objects by great apes and young children
}

Citation for published version (APA):

Barth, J. (2005). Search and exploration of hidden objects by great apes and young children. [Doctoral Thesis, Maastricht University]. Datawyse / Universitaire Pers Maastricht.

https://doi.org/10.26481/dis.20050609jb

Document status and date:

Published: 01/01/2005

DOI:

10.26481/dis.20050609jb

Document Version:

Publisher's PDF, also known as Version of record

\section{Please check the document version of this publication:}

- A submitted manuscript is the version of the article upon submission and before peer-review. There can be important differences between the submitted version and the official published version of record.

People interested in the research are advised to contact the author for the final version of the publication, or visit the DOI to the publisher's website.

- The final author version and the galley proof are versions of the publication after peer review.

- The final published version features the final layout of the paper including the volume, issue and page numbers.

Link to publication

\footnotetext{
General rights rights.

- You may freely distribute the URL identifying the publication in the public portal. please follow below link for the End User Agreement:

www.umlib.nl/taverne-license

Take down policy

If you believe that this document breaches copyright please contact us at:

repository@maastrichtuniversity.nl

providing details and we will investigate your claim.
}

Copyright and moral rights for the publications made accessible in the public portal are retained by the authors and/or other copyright owners and it is a condition of accessing publications that users recognise and abide by the legal requirements associated with these

- Users may download and print one copy of any publication from the public portal for the purpose of private study or research.

- You may not further distribute the material or use it for any profit-making activity or commercial gain

If the publication is distributed under the terms of Article $25 \mathrm{fa}$ of the Dutch Copyright Act, indicated by the "Taverne" license above, 


\section{SEARCH AND EXPLORATION OF HIDDEN OBJECTS BY GREAT APES AND YOUNG CHILDREN}


ISBN 90-5278-469-8

Production: Datawyse / Universitaire Pers Maastricht Coverdesign: Annett Gericke 


\section{SEARCH AND EXPLORATION OF HIDDEN OBJECTS BY GREAT APES AND YOUNG CHILDREN}

\section{PROEFSCHRIFT}

Ter verkrijging van de graad van doctor aan de Universiteit Maastricht, op gezag van de Rector Magnificus, Prof.mr. G.P.M.F. Mols, volgens het besluit van het College van Decanen,

in het openbaar te verdedigen op donderdag 9 juni 2005, om 12.00 uur

door

JOCHEN BARTH

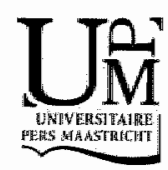




\section{PROMOTOR}

Prof. dr. R. Goebel

\section{CO-PROMOTOR}

Dr. W.G.M. Raaijmakers

\section{BEOORDEELINGSCOMMISIE}

Prof. dr. W.J. Riedel (voorzitter)

Prof. dr. S. Daan (Rijksuniversiteit Groningen)

Prof. dr. B.M. Hood (University of Bristol)

Prof. dr. C. Kemner

Dr. J.E.A. Stauder

The research reported in chapters 1 and 2 was conducted at the Max Planck Institute for Evolutionary Anthropology, Department of Developmental and Comparative Psychology in Leipzig, Germany under the supervision of Prof. Michael Tomasello and Dr. Josep Call. The research reported in chapters 3 and 4 was conducted at the University of Louisiana at Lafayette, Cognitive Evolution Group in Louisiana, USA under the supervision of Prof. Daniel J. Povinelli and James E. Reaux.

The research reported in chapters 1 and 2 and my graduate studentship was supported by the Max Planck Society for which I am utmost grateful.

The support of Universiteit Maastricht is greatly appreciated. 
Für meine Eltern, Gisela und Norbert 


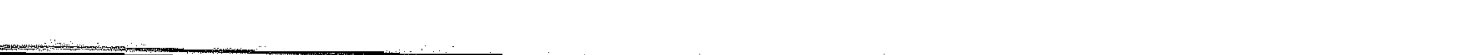




\section{CONTENTS}

Page

Introduction

\section{I - SEARCHING FOR HIDDEN OBJECTS AFTER DISPLACEMENTS}

Chapter 1

Individual and species differences among great apes and young children in a series of object displacement tasks

\section{II - SEARCHING FOR HIDDEN OBJECTS IN THE PRESENCE OF SOCIAL CUES}

Chapter 2

Limitations in the spontaneous use of referential cues by chimpanzees, bonobos, gorillas, and young children

Chaprer 3

Chimpanzees" use of gaze cues in object choice tasks: Different methods yield different results

\section{III - EXPLORING ALARMING OBJECTS BY USING TOOLS}

Chapter 4

Tool-mediated object exploration by chimpanzees: Implications for the evolution and acquisition of human tool-use 
Summary

Samenvatting

Dankwoord

Curriculum vitae

Manuscripts and presentations 
INTRODUCTION 
This volume is comprised of three parts. Each chapter is opened by an introduction to the topic and context of the study. Therefore, in this general introduction I like to suffice with a brief overview of the volume and unfold the background that motivated the set of studies reported here. Additionally, in this introduction I like to highlight the unique aspects of each study. I report four experimental studies and one theoretical paper. The reported research covers parts of three classical areas of developmental psychology: physical cognition, social cognition, and self-awareness. Each topic is presented from a comparative approach including children and great apes. Although this volume covers such diverse areas of cognition, a uniting theme between them is the search and exploration of hidden objects. Subjects in all studies were required to search or explore objects that had been concealed by an experimenter. In Part I, objects were placed under cups and then either remained in the initial location(s) or moved by rotations or displacements. In these tasks, the subjects had to track the spatial relations of the objects and use that physical information to retrieve them. In Part II, the experimenter provided referential cues such as looking towards the location where he hid the object. Subjects were required to use this social information to find the hidden objects. In Part III, I report the implications of an experimental study on tool-use in which chimpanzees used a tool to explore alarming objects. In the last chapter I review comparative research on self-awareness and offer a theoretical elaboration of its evolutionary origins. The study on tool-use and the theoretical chapter add another aspect of searching and exploring objects, namely by the use of tools as opposed to hands. I shall now briefly introduce each of the five chapters.

The first chapter deals with several classical Piagetian tasks involving the retrieval of hidden objects after displacements. We placed three cups on a platform and then administered a series of six tasks to our subjects (1) Delayed Response, (2) Inhibition Test, (3) A-not-B Error, (4) Ratations, (5) Transpositions, (6) Object Permanence. I report the results from 24 great apes and 24 children $2 \frac{1}{2}$ years of age. We were mainly interested in exploring individual and species differences between the four great apes species and children. We found significant differences between the ape species. Also, apes performed significantly better than children on several tasks. A unique aspect of this study is that it unites all four great ape species and children in one study. Additionally, we tested the same number of apes and children. These are two aspects of this study which, to my best knowledge have never been presented before.

The second and the third chapter address the understanding of the referential intent of looking and pointing. Social information signaled by head and eye orientation plays an important role in human and nonhuman daily interaction, because the focus of another individual's attention can serve as a signal to interesting information in the environment. A method of investigating how well animals and children understand the communicative intent of such cues is by the object-choice task. In this task an experimenter hides an interesting object under one of two opaque cups on a table and then gives a cue such as gazing or pointing 
to the baited location. A number of researchers have given this task to a large diversity of populations from children, apes, and dogs to seals and horses with very mixed results. In Chapter 2, I report the results from 17 apes and 24 children of $21 / 2$ years of age. In our study we focused on cues of a solely referential nature. We administered only gaze (head + eyes), glance (eyes only), and point (index finger at chest midline) trials to the subjects. Unlike several previous studies, we deliberately excluded cues that can be interpreted by physical features such as proximity (e.g. tapping). As a new approach, for the children in this study (Experiment 2), I measured the interaction between the subject and the experimenter. For this purpose I used two video cameras and a splitter to record a merged picture of the testing sessions. The recordings allowed me to analyze in detail how the subject reacts to the experimenter's cues. For example, I analyzed how often the children followed my gaze as a direct response to the cue and looked at the same cup as me. This seemed very interesting to investigate as several previous studies have shown that although young children might show an ability to understand the task when it is measured with looking time measures they still show problems when they are asked to respond manually (e.g. searching for an object). In a similar vein, in the experimental design of the child study, I counterbalanced the location of the hidden toy on the last orientation trial and on the first experimental trial per cue. This design allowed me to analyze any potential perseverative reaching errors in the children's responses.

The limitations in the understanding of referential cues that we found in the study with the apes (and children) reported in Chapter 2 made us wonder about the possible causes for the differences in the results reported by Povinelli, Bierschwale, \& Cech (1999). As opposed to our subjects their chimpanzees were clearly able to use an experimenter's gaze direction to locate hidden rewards. After much speculation about the different results and the possible influence of subject history and methodology I followed an invitation from Daniel Povinelli to test the chimpanzees at the Cognitive Evolution Group lab with the methods I had used with the apes in Leipzig and compare this method with the method his group had previously used. In Chapter 3,1 report the results from this study with 5 chimpanzees. We found strikingly different results in the chimpanzees' behavior dependent on the method. The cues were given in the exact same manner in both procedures, the meta-procedures, however, in which the cues were embedded, were different. Differences in the rearing and experimental histories could not explain the differences in the results. The results reported in this chapter demonstrate the important role of the meta-procedures used in the study of social cues. This important issue has been underestimated in previous studies and I think our study made an important contribution by directing attention to the meta-procedures that are seemingly unrelated to the interpretation of social cues, but can influence the interpretation of the results dramatically.

In the fourth chapter I report a study in which chimpanzees received a food-object conflict task. Subjects were introduced to a box that could either 


\section{2 | Introduction}

contain food, an object, or both. The chimpanzees could choose to contact the food or object either with their hands or with a tool as a mediator. Although; I previously reported this study in my Master thesis, the present text has been completely rewritten and the data re-analyzed for publication as a brief report. Moreover, the theoretical framework in which the study is now embedded is further elaborated. In this paper 1 also describe the role of body representation in the evolution of ape and human tool-ase, including the controlled use of fire by early humans.

The last chapter is a theoretical chapter in which several additional ideas that I pursued since my Master's thesis are elaborated. Starting from an extensive summary of self-recognition in children, apes, and other animals, this chapter unfolds a theory of the evolutionary origins of the self based on a kinesthetic selfconcept that enables awareness of the body, actions, and their effect on the environment. 
I

SEARCHING FOR HIDDEN OBJECTS AFTER DISPLACEMENTS 


\section{CHAPTER 1}

\section{Individual and species differences among great apes and young children in a series of object displacement tasks}

Submitted for publication as

Barth, J., \& Call, J. Individual and species differences among great apes (Pan troglodytes, Pan paniscus, Gorilla gorilla, Pongo pygmaeus) and young children (Homo sapiens) in a series of object displacement tasks. 


\begin{abstract}
We administered a battery of object displacement tasks to twenty-four great apes and twenty-four $21 / 2$-year-old children. Objects were placed under one or two of three cups by visible or invisible displacements. The battery included six items: (1) Delayed Response, (2) Inhibition Test, (3) A-not-B, (4) Rotations, (5) Transpositions, (6) Object Permanence. We found significant differences between the species and individuals. In general, the apes performed better than the children on the battery, especially in the transpositions task. Chimpanzees performed significantly better than gorillas and orangutans, especially in the object permanence and rotations task, respectively. All species found non-adjacent trials and rotations especially difficult. The number of elements that changed locations, the type of displacement, and having to inhibit prepotent reaching responses were factors that negatively affected the subjects" performance.
\end{abstract}




\section{INTRODUCTION}

Understanding how objects are represented in the world and what happens to them when they move or disappear is a fundamental part in children's development of understanding the world. In the lives of primates and many other animals keeping track of objects plays an important role, especially in the context of foraging. They have to remember where they have seen food and which location they have already visited and exploited (Call, 2000). Therefore, topics around the representation of objects as operationalized in object permanence and transpositions tasks have after the pioneering ideas of Piaget (1954) received much attention both in developmental and comparative research (Dore \& Dumas, 1987).

In object displacement tasks an object is usually hidden under an opaque cup and then moved with this cup in the presence of other cups. There are two main cognitive challenges in such tasks. First of all, subjects have to understand that objects continue to exist when they are hidden (object permanence) and they have to remember their location until they are given a chance to recover the objects. Numerous paradigms have been used to investigate this ability. For instance, in a delayed response task an object is hidden under one of two or three cups. After a brief delay the subject is then allowed to search for the object. This task goes back to a classic study by Hunter (1913) who tested the memory of animals and children. Later, Harlow and colleagues conducted a series of delayed response studies in which apes and monkeys searched for found that was hidden in one of two locations (e.g., Harlow, 1932). They found that great apes remember the location of food slightly better than old and new world monkeys. In a series of object displacement tasks the delayed response task can function as a baseline measure to determine if subjects understand the basic principles of the task and will search for an object that has been hidden in full view under one cup in the presence of additional cups. Successful retrieval of the object in this task would demonstrate a stage 4 object permanence skill in the subjects. Children typically develop this skill around 8 months of age.

In a more advanced version of this problem, two objects are baited either under the adjacent or non-adjacent cups in an array of three aligning cups. In the non-adjacent condition the center cup is left empty. In a study with orangutans, chimpanzees, and young children on object permanence, Call (2001) found that when subjects start searching for the objects they usually started with the left or right cup and then made a peculiar mistake. After successful retrieval of the first object they proceeded by choosing the middle container, which as they had just seen a few seconds ago was empty. Nevertheless subjects have problems skipping the middle container. This search error is most likely to be explained by an inhibition problem (Call, 2001) rather than a memory deficit.

The second challenge in spatial displacement tasks arises from moving the objects from their initial or usual position to new locations. In the classical Piagetian A-not-B error task an object is placed under the same cup for a number of trials and then in full view of the subject is displaced under another cup. Children 
younger than 12 months have problems recovering the object from the new location even after the visible displacement. As simple as this task is, it is one of the most studied phenomena in developmental psychology. Piaget (1954) initially attributed the error to infants" lack of object permanence and a presumed relation between their own actions and the existence of objects. However, in a classic study, Baillargeon (1987) has shown that infants as young as 4 months look longer at impossible events occurring with hidden objects demonstrating at least an implicit understanding of object permanence. Diamond (1985) has shown that memory seems to play a role in this error as it increases with a delay between hiding and searching. Memory is however not a sufficient factor to explain the search error in location B, as infants still make this error even when they can see the object in the new location (Butterworth, 1977). Perseverative reaching and inhibition errors again seem to play a role in this task.

Displacements are further complicated when they occur invisibly, that is, when the reward changes location by being transported in an opaque container. To solve this task, subjects have to understand that after an object has disappeared under a cup it moves with this cup when the cup is moved. Additionally, subjects have to visually track the movements of objects that are now represented by the cup(s) in the presence of distractions such as irrelevant cups or movements. This means that the spatial representations and positions have to be updated constantly. Three main types of invisible displacements have been investigated depending on what it is being moved: the reward, the reward and the containers, or the reward, containers, and the substrate where the containers (and the reward) rest. Let us begin by discussing the latter.

Rotations consist of an invisible displacement in which an object is first visibly placed under a cup in an array of other cups that are resting on a platform. Then the platform is rotated whereby the object invisibly changes its location. For example, if the left cup of an array of three cups is baited and the platform is rotated by $180^{\circ}$ the object will now be at the opposite location, under the right cup. Beran and Minahan (2000) showed that bonobos and chimpanzees recovered the food after a $180^{\circ}$ rotation well above chance levels in such a task. Beran, Beran, and Menzel (2005) extended those results to $360^{\circ}$ rotations in a study with chimpanzees and also administered $180^{\circ}$ rotations using a 5-container array with two rewards. Subjects were able to select the correct container above chance in their first choice, but not in their second one. Thus, subjects were capable of tracking one of the two items, seemingly the one that was hidden last before the rotation took place. Poti (2000) found that two capuchin monkeys were able to solve a rotations task when the target location was indicated by the presence of a landmark close to the reward, but not when the landmark had to be used independently of the target location. In another study with orangutans and chimpanzees (Call, 2003), the subjects could solve $180^{\circ}$ and $360^{\circ}$ rotations only when they had seen the initial location of the food but not when the location of the reward was signaled by a marker placed on the baited container before the rotation. 
Another invisible object displacement is the transposition task which involves switching the locations of the containers (and the reward inside one of them) while the platform remains stationary. In this task the object is hidden and then moved with the container, whereby two adjacent or non-adjacent containers switch locations in single or double transpositions. For example, when in an array of three cups, the object is placed under the left container and this container switches locations once with the non-adjacent container and then with the adjacent container it will move with the left cup to the outer right position and then to the middle. In a study with 13-month and 21-month old children Sophian and Sage (1983) found that children had problems recovering a toy from the correct container after it had switched locations with another container. In two other studies, Sophian $(1984,1986)$ found evidence that it is not before 3-4 years of age that children solve spatial transpositions. In a study with bonobos and chimpanzees, Beran and Minahan (2000) found that their subjects had no problems solving spatial transpositions with three cups. Call (2003) reported that orangutans and chimpanzees solve spatial transpositions with two cups at ceiling levels. Similarly, Pepperberg, Willner and Gravitz (1997) reported that two African grey parrots passed transposition trials whereas cats and dogs failed them (Doré, Fiset, Goulet, Dumas \& Gagnon, 1996).

The stage 6 object permanence task in the Piagetian series (Piaget, 1954; Sophian \& Sage, 1983; Sophian, 1985) is another invisible displacement task that involves the displacement of the object while both the containers and the substrate remain stationary. In this task a hand or cup is placed over an object in full view of the subject. The object is then moved and displaced under other containers. The experimenter thereby visits either only one container in single displacements, or two containers in double displacements. The object is then secretly left under one of the visited containers and the experimenter shows the subject that she does not have the object anymore. This is the invisible displacement task that has received most research attention (see Doré \& Dumas, 1987; Tomasello \& Call, 1997 for a review). Although the lack of proper control procedures has cast some doubts on the initial comparative studies in this area, currently, there is a consensus that children, great apes, and parrots, unlike other species tested, are capable of solving stage 6 invisible displacements such as double adjacent displacements (Call, 2001; de Blois et al., 1998; Pepperberg et al., 1997). However, 2-year-old children and apes have serious problems choosing the correct container on non-adjacent trials, on which the center cup is left empty (Call, 2001; see also Natale, Antinucci, Spinozzi, \& Poti, 1986; de Blois et al., 1998, for similar results). Call (2001) proposed that an inability to inhibit selecting the container closest to the subject's initial choice, rather than a memory limitation, was at the core of this robust phenomenon.

Despite the information available about the ability to solve spatial displacement tasks in various species, there is an important shortcoming that plagues the comparative literature. Comparisons are the exception rather than the rule in comparative psychology - a problem that occurs both at the species and the 
task level. At the species level, and just focusing on apes, there are very few studies with gorillas and bonobos. For example, there is only one study with a single infant gorilla on object permanience and rotations (Natale et al., 1986, Visalberghi, 1986) and no studies on transpositions. There is a single study on transpositions and rotations in bonobos (Beran \& Minahan, 2000) but no studies on object permanence. Moreover, there are no studies that have compared the performance of various species using the same methods and only three studies that have compared two ape species directly (chimpanzee vs. orangutan and chimpanzee vs. bonobo) in object displacement tasks. This means that we still do not know whether these species differ in their object displacement skills. This lack of comparative data can be extended to human-ape comparisons, which seems ironic given that the human developmental literature was the basis for most of the comparative research in this area.

In addition to the fragmentary picture that arises from bias on particular species, there are very few studies that have tested subjects in more than one task. This has hindered our ability to uncover the cognitive mechanisms that may be underlying the behavior of humans and apes in object displacement tasks. Although the literature offers some hints on what kinds of displacements are the most challenging for subjects, for instance, rotations may be harder than stage 6 displacements which in turn may be harder than transpositions, empirical confirmation is missing.

The purpose of this study was to alleviate these shortcomings by testing all great ape species and young children in a battery of six spatial cognition tasks that had been used in previous studies. These tasks included delayed response, spatial inhibition, A-not-B error, rotations, transpositions, and stage 6 object permanence. The last three tasks included several variations of spatial displacements including single and double invisible displacements. To administer the battery as a whole we reduced the number of trials and administered the tasks in short sessions.

\section{EXPERIMENT 1 : APES}

This experiment investigated the ability of great apes to retrieve hidden food rewards from an array of three cups after various spatial transformations representing various levels of complexity. We manipulated the number, location, and the time that had elapsed since the items were placed under the container as well as the type and number of displacements experimented by the reward. Based on previous research we predicted that introducing a time delay between hiding the reward and letting the subject choose would negatively affect the subjects' performance (e.g. Hunter, 1913). Those trials in which two rewards were placed in adjacent locations would be easier than those in which the rewards were in nonadjacent locations (Call, 2001). Similarly, adjacent displacements in the stage 6 object permanence would be easier than non-adjacent displacements (e.g., Call, 
2001; de Blois et al., 1998; Mendes \& Huber, 2004). Visible displacements in the A-not-B error would be easier than invisible displacements (Natale et al., 1986). Finally, we predicted that transpositions would be easier for the subjects than invisible displacements which in turn would be easier than rotations (Call, 2003). We were especially interested in exploring possible species differences and variations among individuals" ability to solve spatial displacements.

\section{METHOD}

\section{Subjects}

Seven chimpanzees (Pan troglodytes), four bonobos (Pan paniscus), seven gorillas (Gorilla gorilla), and six orangutans (Pongo pygmeaus) participated in this study. All twenty-four subjects were housed at the Wolfgang Köhler Primate Research Center located at the Zoo Leipzig, Germany. Our sample included 8 males and 16 females. There were 10 juveniles (4-8 years of age), and 14 adolescents and adults ( +8 years of age). The mean age was 13,0 years (age range $4-31$ ). All apes were housed in social groups with their conspecifics in spacious indoor and outdoor areas. They were fed three times a day with their species typical diets of vegetables and fruit. Water was available ad libitum. Subjects were never deprived of food and water at any time. All subjects were used to being tested in observation rooms connected to their indoor areas. For a detailed overview of the subjects see 'Table 1 .

\section{Apparatus and materials}

A wooden platform $(82 \mathrm{~cm} \times 60 \mathrm{~cm})$ was attached to a metal frame of a Plexiglas observation window inside the testing-unit (see Figure 1). A panel $(82 \mathrm{~cm}$ $x 30 \mathrm{~cm}$ ) rested on the platform and could be slit back and forward to present the task to the subjects. Two little handles were attached to the platform at the outer sides for allowing a controlled standardized movement with the platform. Three identical opaque square cups (11 cm wide, $8 \mathrm{~cm}$ high) were placed on the panel. In this paper, we will occasionally refer to the cups according to their positions seen from the experimenter's perspective (left, middle, right). For one task (objectpermanence) an additionall little cup ( $6 \mathrm{~cm}$ wide, $5.5 \mathrm{~cm}$ high) was used. Slices of banana and grapes were used as rewards.

\section{Procedure}

The tasks were administered in a similar fashion for Experiment 1 and 2. The locations of the rewards were randomly assigned to the left, middle, and right cup. Subjects received 30 trials in total. The order of the tasks was counterbalanced in blocks. Half of the subjects of each species received the tasks $1+2$ on their first session, tasks $3+4$ on their second session, and tasks $5+6$ on their last session. The other half of the subjects of each species received the tasks in the opposite order per session $2+1,4+3$, and $6+5$. We administered one session per day with the next session typically following on the next day. Subjects were tested individually. We videotaped all trials. 


\section{2 | Chapter 1}

TABLE 1 Age, sex, and rearing history of the subjects that participated in Experiment 1 .

\begin{tabular}{llccc}
\hline Name & Species & Age & Sex & Rearing \\
\hline riaukje & Chimpanzee & 26 & $\mathrm{~F}$ & nursery \\
Jahaga & Chimpanzee & 9 & $\mathrm{~F}$ & mother \\
Gertruida & Chimpanzee & 9 & $\mathrm{~F}$ & mother \\
Fifi & Chimpanzee & 9 & $\mathrm{~F}$ & mother \\
Riet & Chimpanzee & 24 & $\mathrm{~F}$ & nursery \\
Sandra & Chimpanzee & 9 & $\mathrm{~F}$ & mother \\
Frodo & Chimpanze & 8 & $\mathrm{M}$ & mother \\
Ulindi & Bonobo & 8 & $\mathrm{~F}$ & mother \\
Joey & Bonobo & 19 & $\mathrm{M}$ & nursery \\
Limbuko & Bonobo & 6 & $\mathrm{M}$ & nursery \\
Kuno & Bonobo & 5 & $\mathrm{M}$ & nursery \\
Gorgo & Gorilla & 21 & $\mathrm{M}$ & nursery \\
N'Diki & Gorilla & 24 & $\mathrm{~F}$ & unknown \\
N'Kwango & Gorilla & 5 & $\mathrm{M}$ & mother \\
Bebe & Gorilla & 22 & $\mathrm{~F}$ & unknown \\
Ruby & Gorilla & 4 & $\mathrm{~F}$ & mother \\
Viringika & Gorilla & 7 & $\mathrm{~F}$ & mother \\
Vizuri & Gorilla & 7 & $\mathrm{~F}$ & mother \\
Bimbo & Orangutan & 21 & $\mathrm{M}$ & mother \\
Walter & Orangutan & 13 & $\mathrm{M}$ & mother \\
Durija & Orangutan & 31 & $\mathrm{~F}$ & mother \\
Toba & Orangutan & 8 & $\mathrm{~F}$ & mother \\
Pini & Orangutan & 14 & $\mathrm{~F}$ & mother \\
Padana & Orangutan & 4 & $\mathrm{~F}$ & mother \\
& & & & \\
\hline & & & &
\end{tabular}




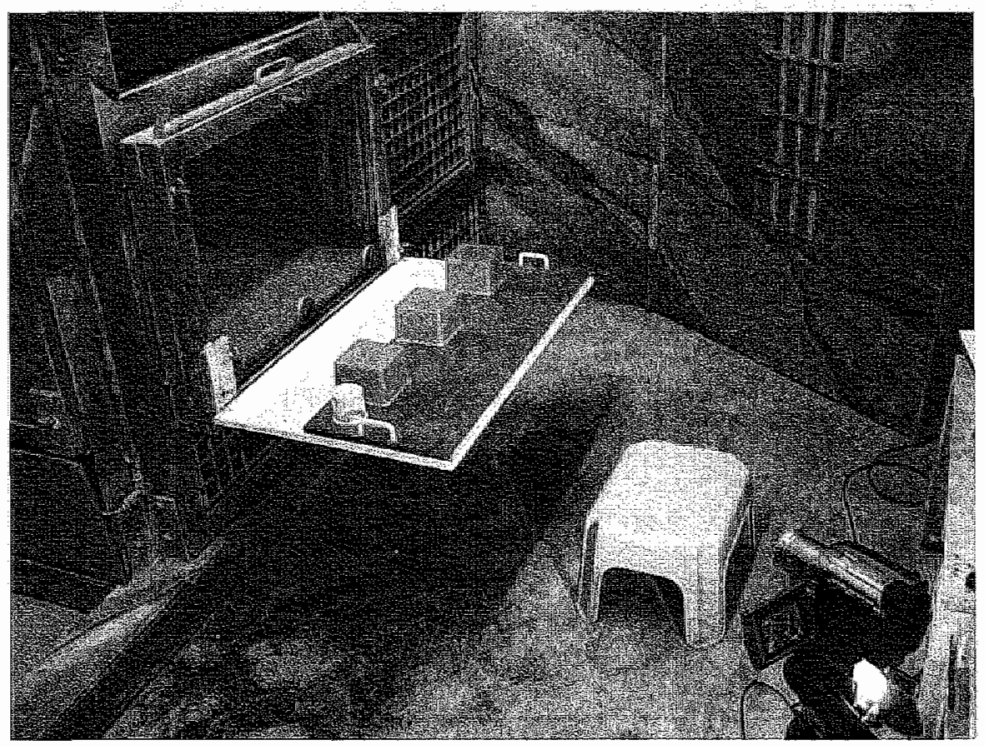

FIGURE I The experimental set-up in Experiment 1. The experimenter and the subject were facing each other. The experimenter mamipulated the cups on the panel and then slid it towards the subject. The apes could choose the cups by touching them through one of the three openings in the Plexiglas panel. 

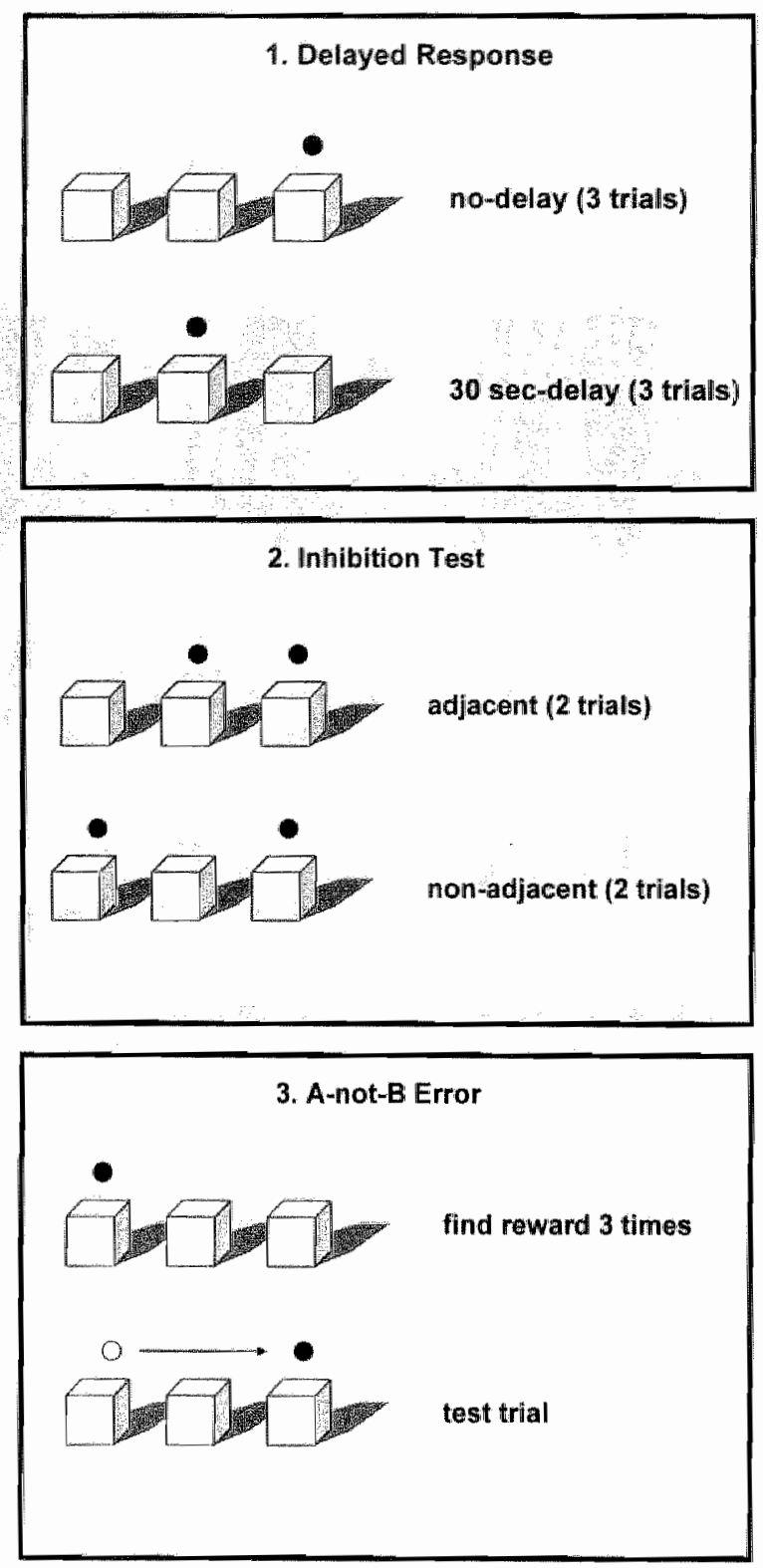

FIGURE 2.1-3 Illustrations of the (1) Delayed Response task, (2) Inhibition Test, (3) and A-not-B task. See text for details. 


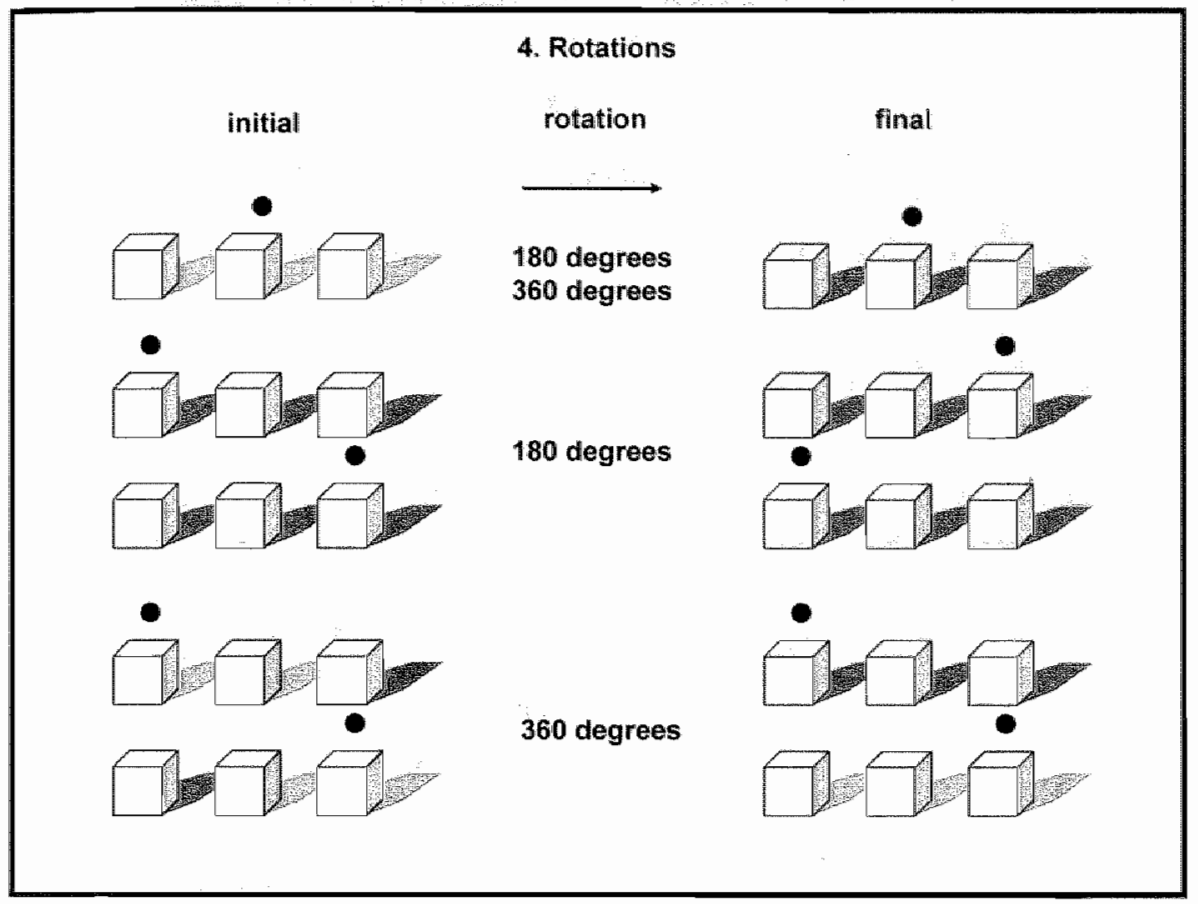

FIGURE 2.4 Illustration of the (4) Rotations task. See text for detaills. 

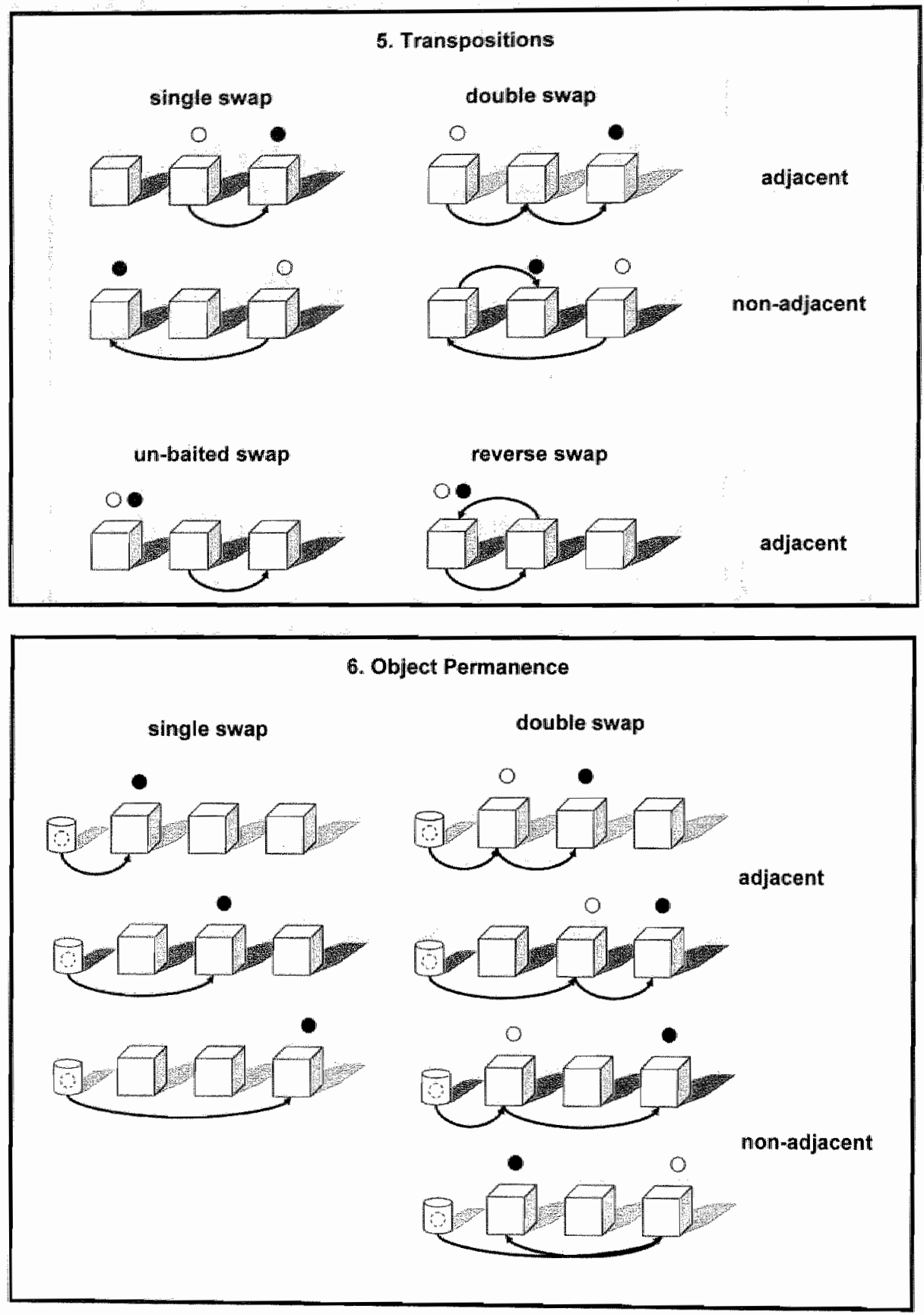

FIGURE 2.5-6 Illustrations of the (5) Transpositions task, and the (6) Object Permanence task. See text for details. 
The experimenter sat behind the table in front of the subject that sat behind the Plexiglas partition with three little holes that allowed the subjects to make a choice by touching the cups on the table. All subjects were very familliar with this arrangement from their participation in other experiments. Upon the onset of each trial the experimenter placed the cups on their side so that the open sides were facing the subject (this will be referred to as open) next to each other on the platform. The platform was in a slit back position to prevent the subjects from making any premature choices. The experimenter then placed, depending on the task, one or to pieces of food in front of one or two cups and placed them upside down concealing the reward(s) (this will be referred to as close). The experimenter then administered the treatment(s) specific to the tasks and pushed the platform against the Plexiglas partition allowing the subject to choose any of the cups. The first cup the subject touched was scored as the choice. If the subject chose the baited cup it received the reward and verbal praise. If the subject chose an un-baited cup the experimenter just lifted the cup showing the subject that it was empty and a new trial was started. We administered the following six tasks (see Figure 2 for illustrations):

Delayed Response ( 6 trials). The experimenter placed one piece of food in front of one of the cups and then closed the cups. Two conditions were administered: (1) no delay, and (2) 30-seconds delay. In the no-delay condition the experimenter pushed the platform against the Plexiglas after he had closed all cups. In the 30seconds delay the experimenter waited for 30 seconds after closing the cups and then pushed the platform forward. During the delay he remained in a neutral motionless posture. Subjects received six trials in total. The first three trials were always no delay trials and the other 30 -seconds delay trials. The location of the food was randomly and exhaustively assigned to the left, middle, and right cup. The 30 seconds were timed by the experimenter with the help of a stopwatch.

Inhibition Test (4 trials). The experimenter placed a piece of food in front of each of two cups and then closed all three cups. There were two different conditions: In (1) adjacent trials the food was placed in front of the center cup and either the left or right cup. In (2) non-adjacent trials the food was placed in front of the left and the right cup, thus leaving the center cup empty. We administered four trials in total, two adjacent and two non-adjacent trials. The sequence of the conditions was randomly assigned.

$A$-not-B (I trial). The experimenter placed a piece of food in front of the left cup; he then closed all cups and pushed the platform forward so the subject could choose a cup. The subject was required to find the reward under the left cup on three successive trials. Once the subject met this criterion the reward was again hidden under the same cup. However, on the critical probe trial the experimenter lifted the cup again and retrieved the reward. In full view of the subject, he then transferred and hid the reward under the right cup. Subjects received one probe trial.

Rotations (6 trials). The experimenter placed a piece of food in front of one of the three open cups. After closing all cups he performed a $180^{\circ}$ rotation or a $360^{\circ}$ 
rotation with the platform. Six trials were given ( 3 cups $\times 2$ conditions). All cups and conditions were randlomly assigned. The conditions produced the following final locations of the reward: after a $180^{\circ}$ rotation the reward is located at the opposite side if the reward had been hidden under either one of the outer cups (left or right). After a $360^{\circ}$ rotation the reward always returns to its starting position. On trials where the reward is placed under the middle cup it remained in the middle position regardless of the rotation.

Transpositions (6 trials). The experimenter placed a piece of food in front of one of the three open cups. After closing all cups he performed one of the following transpositions: (1) single swap, the cup that contained the reward and another either adjacent or non-adjacent empty cup switched locations ( 2 trials), (2) double swap, the cup that contained the reward switched locations with the next adjacent cups thereby moving to the outer location or it switched locations with the non-adjacent cup and then with the adjacent cup thereby returning to the middle location ( 2 trials), (3) reverse swap, the left and the middle cup switched locations two times so that the reward returned to its initial location under the left cup (1 trial), (4) un-baited swap, the two empty cups switched locations, the baited cup was not moved (1 trial).

Object Permanence ( 7 trials). The experimenter closed all cups. Then he placed the reward on the platform to the left of the outer left cup. With his right hand he then took the little white cup and placed it over the reward. He slid the reward with the help of the closed little cup over the panel towards one of the closed cups. With his left hand he carefully lifted a cup thereby only opening the side that is facing him so that the subject cannot see the reward. He made a swapping movement with the white cup as if swapping the reward under the cup. The experimenter moved the reward either just under one cup (single, 3 trials), two adjacent cups (double adjacent, 2 trials), or two non-adjacent cups (double nonadjacent, 2 trials). In the single swap condition the reward remained under this cup, in the double swap conditions the reward is left one time in the first location and one time in the last location that is visited.

\section{Video coding and reliability assessment}

The experimenter coded all trials from the video recordings. For each trial he coded which cup the subject touched first. A second observer coded all trials of two randomly selected subjects of each species (32\% of all total trials). The agreement between the observers was $99 \%$ of the choices (349/352) resulting in a Cohen's $k=.998$.

\section{Data analysis}

First we analyzed the dependent variable mean percent correct of trials with species and task as independent variables. Secondly we analyzed the mean scores for each of the six tasks separately with species and condition as independent variables. Third, we tested the predictions involving comparisons between tasks 
and conditions. Finally, we explored the individual scores for each item. All tests were two-tailed except those involving directional predictions.

\section{RESULTS}

We did not detect any significant effect for order of task presentation and no effect of sex on any of the tasks. Therefore we collapsed these variables in subsequent analyses.

\section{Overall performance}

Table 2 presents the mean percentage of correct trials as a function of species and task. A $4 \times 6$ ANOVA on the percentage of correct responses with species as between-subject factor and task as within-subject factor indicated a significant effect for species, $F(3,20)=6.08, p=0.004$. Bonferroni-Holm post-hoc tests revealed that chimpanzees scored higher than gorillas $(p=0.03)$ and orangutans $(p=0.006)$. There was also a significant effect for condition, $F(5,100)$ $=25.69, p<0.001$. Bonferroni-Holm post-hoc tests revealed that subjects performed significantly better in the A-not-B and transpositions compared to inhibition $(p<0.05)$, object permanence $(p<0.05)$ and rotation $(p<0.001)$. Subjects also performed better in the delayed response compared to inhibition ( $p<$ $0.05)$ and rotation $(p<0.001)$. To summarize, the A-not-B and transposition conditions were the easiest tasks while rotations was the hardest. Object permanence occupied an intermediate position and differed both from the easiest and the hardest task but was equivalent to the delayed response and the inhibition conditions, with those two differing in turn from each other. In addition to the significant main effects of species and task, there was also a significant effect for species $\mathrm{x}$ task, $F(15,100)=1.97, p<0.024$. Next, we analyzed each task separately (see Tables 3 and 4).

TABLE 2 Mean percentage of cortect choices for each task in apes and childrer.

Task

Species

\begin{tabular}{lccccccc} 
& DR & INHIB & A-not-B & ROT & TRANS & OP & Total \\
\hline chimpanzee & 93 & 75 & 100 & 67 & 93 & 88 & 83 \\
bonobo & 88 & 88 & 100 & 29 & 100 & 68 & 93 \\
gorilla & 79 & 54 & 100 & 52 & 81 & 59 & 70 \\
orangutan & 83 & 46 & 83 & 36 & 75 & 71 & 93 \\
apes (total) & 85 & 64 & 96 & 49 & 86 & 72 & 73 \\
children & 76 & 51 & 96 & 47 & 49 & 81 & 63 \\
\hline
\end{tabular}




\section{Individual Tasks}

Delayed Response. Overall subjects selected the correct cup an average of $85 \%$ of the trials which is significantly above chance levels, $t(23)=15.44 ; p<$ 0.001 . A $4 \times 2$ ANOVA investigated the effect of species and condition on the percentage of correct trials (see Table 3). Subjects performed better in the no-delay compared to the delayed condition, $F(1,20)=16.34, p<0.001$. There was no significant effect of species, $F(3,20)=1.0, p=0.41$ or species $\mathrm{x}$ condition, $F(3,20)$ $=2.09, p=0.13$. Apes selected the correct cup significantly more often than would be expected by chance $(p=33)$ in the no-delay condition, $t(23)=18.75 ; p<0.001$ and in the 30-seconds delay condition, $\mathrm{t}(23)=8.37 ; p<0.001$. Inspection of the individual results showed that 22 of the 24 apes seleeted the correct cup on all three trials in the no-delay condition (see Table 4). Ten apes selected the correct cup on all three trials of the 30 -seconds delay condition. The same 10 subjects selected the correct cup on all six trials of the task.

Inhibition Test. Overall subjects selected the correct cup an average of $64 \%$ of the trials which is significantly above chance levels, $\mathrm{t}(23)=5.57 ; p<0.001$ (see Table 3). A $4 \times 2$ ANOVA investigated the effect of species and condition on the percentage of correct trials (see Table 3 ). Subjects performed significantly better in the adjacent compared to the non-adjacent condition, $F(1,20)=9.79, p=$ 0.005 . Additionally, there were significant differences across species, $F(3,20)=$ $3.70, p=0.029$, but no species $\mathrm{x}$ condition, $F(3,20)=2.09, p=0.13$. However, after employing the Bonferroni-Holm post-hoc test, we could not confirm that bonobos outperformed orangutans $(p=0.06)$. There was also no significant effect of species $\mathrm{x}$ condition, $F(3,20)=1.38, p=0.28$. Thus, we decided to analyze the data of all subjects together. The apes selected the correct cup significantly more often than would be expected by chance $(p=33)$ in the adjacent condition, $t(23)=$ $7.31 ; p<0.001$, but not in the non-adjacent condition, $\mathrm{t}(23)=1.52 ; p=0.14$. We then compared the subjects' first and second choices depending on the conditions. On adjacent trials, subjects were correct on their first choice on $92 \%$ of the trials and on $89 \%$ of their second choices. On non-adjacent trials, subjects were correct on their first choice on $79 \%$ of the trials, but only on $58 \%$ of their second choices. An inspection of the individual data showed that 17 of the 24 apes chose the correct cups on both adjacent trials, whereas only 7 chose the correct cups on both nonadjacent trials (see Table 4). Only 6 apes chose the correct cups on all four trials

$A-n o t-B$. Overall subjects selected the correct cup an average of $96 \%$ of the trials which is significantly above chance levels, $\mathrm{t}(23)=15.00 ; p<0.001$ (see Table 3). In fact, only one subject (Dunja) missed the correct cup on the transposition trial. Because of this ceiling effect we conducted no further analyses. 
TABLE 3 Mean percentage of correct choices per species for each task and condition for Experiment 1 and 2 .

\begin{tabular}{|c|c|c|c|c|c|c|}
\hline \multirow[b]{2}{*}{$\begin{array}{l}\text { Task / } \\
\text { Condition }\end{array}$} & \multicolumn{6}{|c|}{ Species } \\
\hline & $\begin{array}{c}C \\
n=7\end{array}$ & $\begin{array}{c}B \\
n=4\end{array}$ & $\begin{array}{c}\mathrm{G} \\
\mathrm{n}=7\end{array}$ & $\begin{array}{c}0 \\
n=6\end{array}$ & $\begin{array}{c}A \\
n=24\end{array}$ & $\begin{array}{c}H \\
n=24\end{array}$ \\
\hline Delayed Response (6) & 93 & 88 & 79 & 83 & 85 & 76 \\
\hline $0(3)$ & 100 & 92 & 86 & 100 & 94 & 92 \\
\hline $30(3)$ & 86 & 83 & 71 & 61 & 75 & 60 \\
\hline Inhibition Test (4) & 75 & 88 & 54 & 46 & 64 & 51 \\
\hline adjacent (2) & 93 & 100 & 86 & 50 & 81 & 67 \\
\hline non-adjacent (2) & 57 & 75 & 21 & 42 & 46 & 33 \\
\hline A-not-B Error (1) & 100 & 100 & 100 & 83 & 96 & 96 \\
\hline Rotations (6) & 67 & 29 & 52 & 36 & 49 & 47 \\
\hline $180(3)$ & 71 & 31 & 43 & 42 & 49 & 50 \\
\hline $360(3)$ & 61 & 31 & 68 & 25 & 49 & 60 \\
\hline $180-\mathrm{LR}(2)$ & 57 & 12 & 43 & 50 & 44 & 21 \\
\hline $360-\operatorname{LR}(2)$ & 79 & 38 & 50 & 33 & 52 & 46 \\
\hline $180-M(1)$ & 86 & 50 & 43 & 33 & 54 & 79 \\
\hline $360-M(1)$ & 43 & 25 & 86 & 17 & 46 & 75 \\
\hline Transpositions (6) & 93 & 100 & 81 & 75 & 86 & 49 \\
\hline single (2) & 100 & 100 & 79 & 67 & 85 & 33 \\
\hline double (2) & 79 & 100 & 79 & 67 & 79 & 38 \\
\hline reverse (1) & 100 & 100 & 71 & 83 & 88 & 71 \\
\hline un-baited (1) & 100 & 100 & 100 & 100 & 100 & 75 \\
\hline Object Permanence $(7)$ & 88 & 68 & 59 & 71 & 72 & 81 \\
\hline single (3) & 100 & 75 & 71 & 78 & 82 & 93 \\
\hline double-adjacent (2) & 93 & 75 & 64 & 75 & 77 & 92 \\
\hline double-non-adjacent (2) & 64 & 50 & 43 & 58 & 54 & $\$ 4$ \\
\hline Total Mear & 84 & 74 & 67 & 64 & 73 & 63 \\
\hline
\end{tabular}

Note $\mathrm{C}=$ Chimpanzee, $\mathrm{B}=\mathrm{Bonobo}, \mathrm{G}=$ Gorilla, $\mathrm{O}=$ Orangutan, $\mathrm{A}=\mathrm{Ape}, \mathrm{H}=$ Human. The number of trials is indicated in brackets. 
TABLE 4 Number of indiwduals per species who selected the correct cup on all trials per task and condition in Experiment $\mathbb{I}$ and 2 .

\section{Species:}

\begin{tabular}{|c|c|c|c|c|c|c|}
\hline $\begin{array}{l}\text { Trask / } \\
\text { Condition }\end{array}$ & $\begin{array}{c}C \\
n=7\end{array}$ & $\begin{array}{c}B \\
n=4\end{array}$ & $\begin{array}{c}\mathrm{G} \\
\mathrm{n}=7\end{array}$ & $\begin{array}{c}0 \\
n=6\end{array}$ & $\begin{array}{c}A \\
n=24\end{array}$ & $\begin{array}{c}H \\
n=24\end{array}$ \\
\hline Dellayed Response (6) & 4 & 2 & 2 & 2 & 10 & 5 \\
\hline $0(3)$ & 7 & 3 & 6 & 6 & 21 & 20 \\
\hline $30(3)$ & 4 & 2 & 2 & 2 & 10 & 5 \\
\hline Inhibition Test (4) & 3 & 2 & 1 & 0 & 6 & 1 \\
\hline Adjaeent (2) & 6 & 4 & 5 & 2 & 17 & 9 \\
\hline non-adjacent (2) & 3 & 2 & 1 & 1 & 7 & 4 \\
\hline A-not-B Exror (1) & 7 & 4 & 7 & 5 & 23 & 23 \\
\hline Rotation (6) & 1 & 0 & 0 & 0 & $\mathbb{1}$ & 0 \\
\hline $180(3)$ & 3 & 0 & 0 & 0 & 3 & 1 \\
\hline $360(3)$ & 2 & 0 & 0 & 0 & 2 & 6 \\
\hline 180-LR (2) & 3 & 0 & 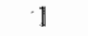 & 2 & 6 & 1 \\
\hline $360-\mathrm{LR}(2)$ & 4 & 0 & 0 & 1 & 5 & 7 \\
\hline $180-M(1)$ & 6 & 2 & 3 & 2 & 13 & 19 \\
\hline $360-\mathrm{M}(1)$ & 3 & 1 & 6 & $\mathbb{1}$ & 11 & 18 \\
\hline Transpositions (6) & 4 & 4 & 2 & 1 & 11 & 0 \\
\hline single (2) & 7 & 4 & 4 & 2 & 17 & 4 \\
\hline double (2) & 4 & 4 & 4 & 3 & 15 & 3 \\
\hline reverse (1) & 7 & 4 & 5 & 5 & 21 & 17 \\
\hline un-baited (1) & 7 & 4 & 7 & 6 & 24 & 18 \\
\hline Object Permanence (7) & 2 & 0 & 0 & 0 & 2 & 5 \\
\hline single (3) & 7 & 2 & 3 & 3 & 15 & 20 \\
\hline double-adjacent (2) & 6 & 2 & 3 & 4 & 15 & 20 \\
\hline double-noin-adjacent (2) & 2 & 1 & 0 & 1 & 4 & 6 \\
\hline
\end{tabular}
Note. $\mathrm{C}=$ Chimpanzee, $\mathrm{B}=\mathrm{Bonobo}, \mathrm{G}=$ Gorilla, $\mathrm{O}=$ Orangutan, $\mathrm{A}=\mathrm{Ape}, \mathrm{H}=$ Human. The number
of trials is indicated in brackets. 
Rotations. Overall subjects selected the correct cup an average of $49 \%$ of the trials which is significantly above chance levels, $\mathrm{t}(23)=3.41 ; p=0.002$. A $4 \mathrm{x}$ 4 ANOVA investigated the effect of species and condition on the percentage of correct trials (see Table 3 ). There were significant differences across species, $F(3,20)=4.19, p=0.019$, but no effect of condition, $F(3,60)=0.45, p=0.71$, or species $\mathrm{x}$ condition, $F(9,60)=1.39, p=0.21$. Bonferroni-Holm post-hoc tests revealed that chimpanzees outperformed orangutans $(p=0.048)$. We therefore decided to analyze the data separately by species. The chimpanzees selected the correct cup significantly more often than would be expected by chance $(p=33)$ in the $180^{\circ}$ condition, $\mathrm{t}(6)=3.347 ; p=0.015$; but not in the $360^{\circ}$ condition, $\mathrm{t}(6)=$ $2.305 ; p=0.061$. The gorillas showed the opposite pattern. They selected the correct cup significantly more often than would be expected by chance $(p=33)$ in the $360^{\circ}$ condition, $\mathrm{t}(6)=4.880 ; p=0.003$; but not in the $180^{\circ}$ condition, $\mathrm{t}(6)=$ $0.938 ; p=0.385$. Bonobos and orangutans did not select the correct cup above chance levels in any of the conditions. When we considered the position of the reward for each condition we found that chimpanzees selected the correct cup above chance levels in the $180^{\circ}$ condition only when it was located in the middle, $\mathrm{t}(6)=3.690 ; p=0.010$, but not when it was under the left or right cup, $t(6)=1.420$; $p=0.205$. In the $360^{\circ}$ condition the chimpanzees found the reward above chance level when it was hidden under the left or right cup, $\mathrm{t}(6)=4.511 ; p=0.004$, but did not find the reward above chance levels when it was located in the middle, $t(6)=$ $0.488 ; p=0.643$. In contrast, the gorillas selected the correct cup in the $360^{\circ}$ condition more often than expected by chance, $\mathrm{t}(6)=3.690 ; p=0.010$. Inspection of the individual data revealed that only one subject (Jahaga) selected the correct cup on all six trials (see Table 4). Additionally, two chimpanzees (Frodo, Sandra) selected the correct cup on all three trials of the $180^{\circ}$ condition. One chimpanzee (Fifi) selected the correct cup on all three trials of the $360^{\circ}$ condition. No other subject selected the correct cup on more than 2 trials per condition.

Transpositions. Overall subjects selected the correct cup an average of $86 \%$ of the trials which is significantly above chance levels, $t(23)=16.92 ; p<$ 0.001 . A $4 \times 4$ ANOVA investigated the effect of species and condition on the percentage of correct trials (see Table 3 ). Since sphericity could not be assumed for the condition factor (Mauchly's $\mathrm{W}=0.233 \mathrm{df}=5, p<0.001$ ) we adjusted the degrees of freedom using the Huynh-Feldt index. There were significant differences across species, $F(3,20)=3.62, p=0.031$, but no effect of condition, $F(2,38)=2.48$, $p=0.099$, or species $\mathrm{x}$ condition, $F(6,38)=0.89, p=0.51$. However, after employing the Bonferroni-Holm post-hoc test, we could not confirm that bonobos outperformed orangutans $(p=0.09)$. Thus, we decided to analyze the data together. The apes selected the correct cup significantly more often than would be expected by chance $(p=33)$ in all conditions; single swap $t(23)=11.061 ; p<0.001$, double swap, $\mathrm{t}(23)=7.751 ; p<0.001$, reverse swap, $\mathrm{t}(23)=7.903 ; p<0.001$. Additionally, on the un-baited swap trial all 24 subjects chose the correct cup. Individual analyses indicated that 11 of the 24 apes chose the correct cup on all trials (see Table 4 ). 
Object Permanence. Overall subjects selected the correct cup an average of $72 \%$ of the trials which is significantly above chance levels, t $(23)=10.20 ; p<$ 0.001 . Even if we use the more conservative value of $p=50$ associated with double displacements, subjects still performed above chance, $t(23)=5.80 ; p<0.001$. A 4 $x=3$ ANOVA investigated the effect of species and condition on the percentage of correct trials (see Table 3). There was a significant effect of condition, $F(2,40)=$ $6.37, p=0.004$, and a nearly significant effect of species, $F(3,20)=3.08, p=0.051$ and no effect of species $\mathrm{x}$ condition, $F(6,40)=0.12, p=0.99$. Bonferroni-Holm post-hoc tests indicated that chimpanzees outperformed gorillas $(p=0.048)$. Moreover, Bonferroni-Holm post-hoc tests indicated that subjects performed worse in the double non-adjacent condition compared to the single displacement condition $(p=0.003)$ and the double adjacent condition $(p=0.046)$. Subjects selected the correct cup significantly more often than would be expected by chance $(p=50)$ in the single displacement condition, $t(23)=6.03 ; p<0.001$ and in the double adjacent condition, $t(23)=4.03 ; p=0.001$, but not in the double non-adjacent condition, $\mathrm{t}(23)=0.81 ; p=0.43$. Individual analyses indicated that only 2 of the 24 apes (Jahaga and Frodo) chose the correct cup on all seven trials (see Table 4). Fifteen apes chose the correct cup on both single swap trials; also 15 apes chose the correct cup on both adjacent double swap trials. In contrast, only 4 subjects chose the correct cup on both non-adjacent double swap trials.

\section{Testing the predictions}

The analysis of the Delayed response task had already indicated that the delayed condition was significantly harder than the non-delayed condition $(p<$ 0.001 ), thus confirming our first prediction. The ceiling effect observed in the Anot-B task also indicated this task was easier than invisible displacements, thus confirming our third prediction. Next we analyzed the remaining predictions. First, we analyzed the effect of trajectory and displacement on the percentage of correct responses by directly comparing the two conditions of the inhibition tests and the two double displacements conditions in the object permanence test. A $3 \times 2 \times 2$ ANOVA with species as between-subject factor and trajectory (adjacent vs. nonadjacent) and displacement (yes vs. no) as within-subject factors indicated that nonadjacent conditions were harder than adjacent conditions, $F(1,20)=11.92, p=$ 0.003 and that there was also an effect of species, $F(3,20)=4.21, p=0.018$. No other factors or interactions were significant $(p>0.17$ in all cases). BonferroniHolm post-hoc tests revealed that chimpanzees scored significantly higher than
gorillas $(p=0.048)$.

Second, we analyzed the effect of type of displacement on the percentage of correct responses by directly comparing the double transposition, the stage 6 double adjacent displacement, and the $180^{\circ}$ rotation -all those displacements
involved a change in the between-subject factor and displacement type (transposition and rotation) as within-subject factor revealed (transposition, invisible displacement, 
$F(2,40)=8.50, p=0.001$ and no effect for species, $F(3,20)=1.05, p=0.39$ or species $\mathrm{x}$ condition, $F(6,40)=1.05, p=0.41$. Bonferroni-Holm post-hoc tests revealed that the rotation was significantly harder than the transposition $(p=0.006)$ and the invisible displacement $(p=0.006)$. Contrary to our prediction, double displacement transpositions were not easier than dowble invisible displacements ( $p$ $=1.0 \%$.

\section{DISCUSSION}

Overall chimpanzees outperformed gorillas and orangutans (but not bonobos) in the current battery of tasks. These differences were particularly evident in those tasks involving some sort of object displacement. Thus, chimpanzees outperformed gorillas in object permanence and orangutans in rotations. This latter result differs from a previous study that found no significant differences between chimpanzees and orangutans (Call, 2003).

In general, the results confirmed our predictions. Transpositions were the easiest task followed by invisible displacements and rotations, which were substantially harder then other tasks. The visible displacement in the A-not-B task presented no problems for subjects and it was easier than single invisible displacements. Adjacent trials were easier than non-adjacent trials independently of whether there was a displacement involved. Finally, the delayed condition was harder than its non-delayed counterpart.

Apes passed all the conditions except those involving non-adjacent trials (with or without displacements) and various kinds of rotations. These results replicate previous findings (e.g., Call, 2001, 2003; de Blois et a1., 1998) and extend the results to bonobos and gorillas. The difficulty experimented with non-adjacent trials replicated the findings of previous studies both with and without displacements (e.g., Natale et al., 1986; Call, 2001; de Blois et al., 1998). The current study also confirmed that rotations were the hardest kind of displacement, a finding that the results of a previous study suggested but was not tested directly (Call, 2003). Nevertheless, the current study showed much lower levels of success in rotations than previously reported. Call (2003) found that chimpanzees and orangutans responded correctly in $81 \%$ of the trials in the $180^{\circ}$ rotation condition whereas in the current study subjects were correct in only $54 \%$ of the trials. Note that Beran and Minahan (2000) also found that chimpanzees and bonobos performed at high levels. A possible explanation for the discrepancy between the current and previous results is the number of cups presented to the subjects. Previous studies used two cups while we used three cups in the current study.

\section{EXPERIMENT 2 : CHILDREN}

We tested 2.5 year-old children with the same battery of tasks that we administered to the apes in the previous experiment. Based on previous studies we had similar 
predictions as for the apes in Experiment 1 except that we predicted that young children will have problems solving transposition tasks (Sophian, 1984; 1986).

\section{METHOD}

\section{Paricipants}

Twenty-four children (12 females, 12 males) of $2.5-2.7$ years of age (mean $\left.=2 \frac{1}{2}\right)$ from Leipzig, a mid-sized town in Germany, participated in this study. The majority of the children come from a German speaking middle class background. They were recruited from a database that is maintained in collaboration with local Kindergartens. The parents had approved their children's participation in written form. All children were tested at the local Kindergartens in Leipzig, Germany. The parents regularly received information materials about ongoing studies at the child lab.

\section{Apparatus and materials}

Children were seated at a children's table that was set up in the playroom of their kindergarten. A wooden platform $(78 \mathrm{~cm} \times 30 \mathrm{~cm})$ was used to present the tasks to the children. Stamps with motives of cartoon characters that children could stamp on a sheet of paper were used as rewards (see Figure 3). Three identical square cups ( $11 \mathrm{~cm}$ wide, $8 \mathrm{~cm}$ high) were used to hide the stamp(s). The cups were identical in size and appearance to the cups used with the apes in Study 1. Again, we will occasionally refer to the cups according to their positions seen from the experimenter's perspective (left, middle, right). For one task (object-permanence) an additional little cup (about $6 \mathrm{~cm}$ wide and $5 \mathrm{~cm}$ high) was used.

\section{Procedure}

The tasks were administered in a similar fashion as in Experiment 1. All subjects were tested individually with only the experimenter, an assistant, and the subject in the room. The experimenter sat on the floor behind the table facing the subject. The child was seated in the small chair in front of the table facing the experimenter. All trials were recorded on video.

\section{Fideo coding and reliability assessment}

The experimenter coded all trials from the video recordings. For each trial the coded which cup the subject touched first. A second observer coded all trials of eight randomly selected subjects, four boys and four girls ( $32 \%$ of total trials). The agreement between the observers was calculated by using Cohen's Kappa. The observers agreed on $98 \%$ of the trials $(367 / 375)$ resulting in a Kappa value of $k$ $=.97$. 


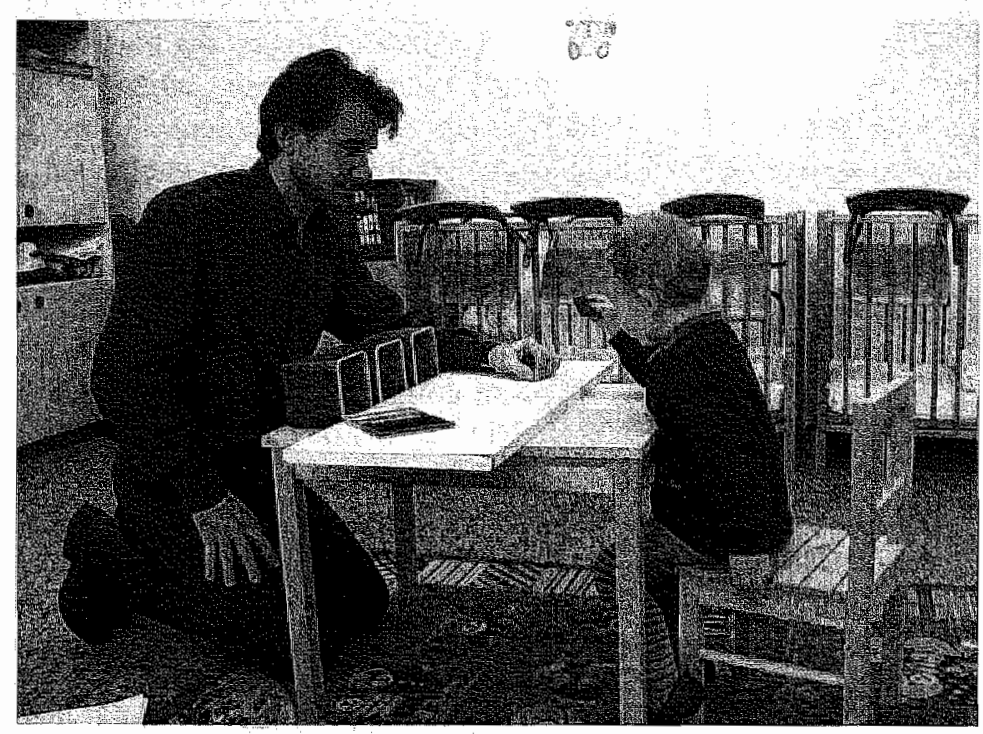

FIGURE 3 The experimental set-up in Experiment 2. The experimenter and the subject were facing each other. The experimenter manipulated the cups on the panel and then slid it towards the subject. The children could lift one of the cups and, when it contained a stamp, stamp on a piece of paper.

\section{Data analysis}

As for the apes in Experiment 1 we analyzed all data at the group level (all subjects). First we analyzed the overall mean scores; secondly we analyzed the mean scores for each of the six items. Finally, we compared the conditions of each task. Additionally, we explored the individual scores for each item.

\section{RESULTS}

There was no effect for order of task presentation and no effect of gender on any of the tasks. Therefore we analyzed the data of all 24 children together.

\section{Overall performance}

Children chose the correct cup on $63 \%$ of the experimental trials (see Table 2). An ANOVA on the percentage of correct responses with condition as a withinsubject factor indicated a significant effect for condition, $F(5,115)=28.50, p<$ 0.001 . Bonferroni-Holm post-hoc tests indicated that subjects performed significantly better in the A-not-B condition compared to all other conditions ( $p<$ 
0.025). In addition, subjects performed significantly better in the object permanence and delayed response conditions compared to inhibition, transposition, and rotation $(p<0.001$ in all cases). No other comparisons reached a significant difference. This means that the A-not-B condition was the easiest condition followed by object permanence and delayed response whereas inhibition, transposition, and rotation were the hardest (in ascending order). Table 3 presents the percentage of correct trials for the various conditions within each task.

\section{Individual Tasks}

Delayed Response. Overall subjects selected the correct cup an average of $76 \%$ of the trials which is significantly above chance levels, $t(23)=11.51 ; p<$ 0.00 I. An ANOVA investigated the effect of condition on the percentage of correct trials (see Table 3). Subjects performed better in the no-delay compared to the delayed condition, $F(\mathbb{I}, 23)=29.89, p<0.001$. Children selected the correct cup significantly more often than would be expected by chance in the no-delay condition, $t(23)=14.11 ; p<0,001$ and in the 30 -seconds delay condition, $t(23)=$ $4.99 ; p<0.001$. Inspection of the individual results showed that 20 of the 24 children selected the correct cup on all three trials in the no-delay condition (see Table 4). Five children selected the correct cup on all three trials of the 30 -seconds delay condition. The same 5 subjects selected the correct cup on all six trials of the task.

Inhibition Test. Overall subjects selected the correct cup an average of $51 \%$ of the trials which is significantly above chance levels, $\mathrm{t}(23)=3.83 ; p=0.001$. An ANOVA investigated the effect of condition on the percentage of correct trials (see Table 3 ). Subjects performed significantly better in the adjacent compared to the non-adjacent condition, $F(1,23)=10.51, p=0.004$. Children selected the correct cup significantly more often than would be expected by chance in the adjacent condition, $t(23)=5.79 ; p<0.001$; but not in the non-adjacent condition, $t(23)=0.004 ; p=0.98$. We then compared the subjects" first and second choices depending on the conditions. On adjacent trials, subjects were correct on their first choice on $85 \%$ of the trials, and on $76 \%$ of their second choices. On non-adjacent trials, subjects were correct on their first choice on $50 \%$ of the trials, and on $67 \%$ of their second choices. An inspection of the individual data showed that 9 of the 24 children chose the correct cups on both adjacent trials, whereas only 4 chose the correct cups on both non-adjacent trials (see Table 4). Only one child chose the correct cups on all four trials.

$A-$ not $-B$. Overall subjects selected the correct cup an average of $96 \%$ of the trials which is significantly above chance levels, $\mathrm{t}(23)=15.00 ; p<0.001$ (see Table 3). Only one child missed the correct cup on the probe trial,

Rotations. Overall subjects selected the correct cup an average of $47 \%$ of the trials which is significantly above chance levels, $\mathrm{t}(23)=4.25 ; p<0.001$. An ANOVA investigated the effect of condition on the percentage of correct trials (see Table 3). There were significant differences across conditions, $F(3,69)=10.06, p<$ 
0.001. Bonferroni-Holm post-hoc tests revealed that children performed significantly worse in the $180^{\circ}$ rotation with change in the reward position compared to the $180^{\circ}$ and $360^{\circ}$ rotations without change in the reward position. Children selected the correct cup significantly more often than would be expected by chance when the reward remained in the same location throughout the trial (180" $\left.\mathrm{t}(23)=5.42 ; p<0.001 ; 360^{\circ}: \mathrm{t}(23)=4.62 ; p<0.001\right)$ but not when the reward changed positions. In fact, they performed below chance in the $180^{\circ}$ condition, $t(23)$ $=2.09 ; p=0.048$, and at chance level in the $360^{\circ}$ condition, $\mathrm{t}(23)=1.48 ; p=0.152$ ). Inspection of the individual data revealed that only one subject selected the correct cup on all three trials of the $180^{\circ}$ condition (see Table 4). Six children selected the correct cup on all three trials of the $360^{\circ}$ condition. No subject selected the correct cup on all six trials.

Transpositions. Overall subjects selected the correct cup an average of $49 \%$ of the trials which is significantly above chance levels, $\mathrm{t}(23)=3.51 ; p=0.002$. An ANOVA investigated the effect of species and condition on the percentage of correct trials (see Table 3 ). There were significant differences across conditions, $F(3,69)=6.86, p<0.001$. Bonferroni-Holm post-hoc tests revealed that children performed significantly better in the unbaited swap compared to the single swap ( $p$ $=0.006)$ and the double swap $(p=0.024)$. Similarly, children performed significantly better in the reverse swap compared to the single swap $(p=0.027)$ and the double swap $(p=0.015)$. Children selected the correct cup significantly more often than would be expected by chance in the reverse swap, $\mathrm{t}(23)=3.96 ; p=0.001$ and in the un-baited swap, $\mathrm{t}(23)=4.62 ; p<0.001$, but not after the single swap, $\mathrm{t}(23)=0.00 ; p=1.0$, or the double swap, $\mathrm{t}(23)=0.61 ; p=0.55$. Individual analyses indicated that none of the 24 children chose the correct cup on all trials (see Table 4). Only four children chose the correct cup on more than $80 \%$ of the trials.

Object Permanence. Overall subjects selected the correct cup an average of $89 \%$ of the trials which is significantly above chance $(p=50), t(23)=10.54 ; p<$ 0.001 . An ANOVA investigated the effect of condition on the percentage of correct trials (see Table 3). There was a significant effect of condition, $F(2,46)=22.35, p<$ 0.001 . Bonferroni-Holm post-hoc tests indicated that subjects performed worse in the double non-adjacent condition compared to the single displacement $(p<0.001)$ and the double adjacent condition $(p<0.001)$. Subjects selected the correct cup significantly more often than would be expected by chance in the single displacement condition, $\mathfrak{t}(23)=17.26 ; p<0.001$ and in the double adjacent condition $(p=50), \mathrm{t}(23)=10.72 ; p<0.001$, but not in the double non-adjacent condition, $\mathrm{t}(23)=0.62 ; p=0.54$. Individual analyses indicated that only 5 of the 24 children chose the correct cup on all seven trials (see Table 4). Twenty children chose the correct cup on both single swap trials; also 20 children chose the correct cup on both adjacent double swap trials. In contrast, only 6 subjects chose the correct cup on both non-adjacent double swap trials. 


\section{Testing the predictions}

The analysis of the Delayed response task had already indicated that the delayed condition was significantly harder than the non-delayed condition $(p<$ $0.001)$, thus confirming our first prediction. Likewise, the ceiling effect observed in the $A$-not-B task also indicated that this task was easier than invisible displacements thus confirming our third prediction. Next we analyzed the remaining predictions. First, we analyzed the effect of trajectory and displacement on the percentage of correct responses by directly comparing the two conditions of the inhibition tests and the two double displacements conditions in the Object permanence task. A $2 \times$ 2 ANOVA with trajectory (adjacent vs. non-adjacent) and displacement (yes vs. no) as within-subject factors indicated that the adjacent conditions were easier than the non-adjacent conditions, $F(1,23)=21.37, p<0.001$. Additionally, the presence of a displacement made the task easier, $F(1,23)=16.67, p<0.001$. The trajectory $x$ displacement was not significant, $F(1,23)=0.19, p=0.66$.

Second, we analyzed the effect of type of displacement on the percentage of correct responses by directly comparing the double transposition, the stage 6 double adjacent displacement, and the $180^{\circ}$ rotation - all those displacements involved a change in the position of the reward. A repeated measures ANOVA with displacement type (transposition, invisible displacement, and rotation) as within-subject factor revealed a significant effect for condition, $F(2,46)=49.11, p<$ 0.001 . Bonferroni-Holm post-hoc tests revealed that invisible displacements were significantly easier than rotations $(p<0.001)$ and transpositions $(p<0.001)$. Additionally, transpositions were significantly easier than rotations $(p=0.043)$.

\section{Comparison between Apes and Children}

Table 2 shows the mean percentage of correct trials for each task in apes and children. A $2 \times 6$ ANOVA on the percentage of correct responses with species (apes vs. children) as between-subject factor and task as within-subject factor indicated a significant effect for species, $F(3,20)=6.08, p=0.004$, task, $F(5,100)$ $=25.69, p<0.001$, and species $\mathrm{x}$ task, $F(15,100)=1.97, p<0.024$. Therefore, we cornpared apes and children in each task separately. Apes outperformed children in the Transpositions task, $\mathrm{t}(46)=6.992 ; p<0.001$. In contrast, there were no significant differences between apes and children on the other tasks $(p>0.30$ in all cases).

\section{DISCUSSION}

Overall apes outperformed 2.5-year-old children in the current battery of tasks, although the main difference resided on the Transposition task in which apes clearly outperformed children ( $86 \%$ vs. $49 \%$ correct trials, respectively). In general, the results confirmed our predictions. Visible displacements in the A-not-B task presented no problems for subjects and it was the easiest task of all (e.g., Piaget, 1952). As expected, non-delayed trials were also easier than delayed trials. A comparison between the trials in the Inhibition and the Object permanence tasks 
revealed that adjacent trials were easier than non-adjacent trials independently of whether there was a displacement involved, thus confirming previous results (Call, 2001). Interestingly, both adjacent and non-adjacent trials were easier when there was a displacement involved. Although this may seem counterintuitive, one has to recall that inhibition trials (which involve no displacement) have an expected probability of 0.33 whereas double invisible trials (which involve a displacement) have an expected probability of 0.50 .

As it was the case with the apes, children found rotations particularly difficult. Additionally, unlike the apes, children found transpositions harder than object permanence displacements. Children experienced the greatest difficulties when the reward changed its initial position due to the rotation or transposition of cups. These results confirm previous studies with children that had suggested that rotations were the hardest type of displacement and object permanence displacements were the easiest (Lasky et al., 1980; Sophian, 1984, 1985). In fact, Lasky et al. (1980) found that children younger than seven years of age find rotations particularly difficult.

\section{GENERAL DISCUSSION}

Apes and young children showed proficiency in solving various kinds of object displacements. Although overall apes performed better than the children, a separate analysis for each task did not detect significant differences between apes and children except for transpositions. Focusing on apes, we found significant differences between the species. Overall, chimpanzees performed better than gorillas and orangutans but not better than bonobos.

Several implications can be drawn from our results regarding the factors that control the successful search of objects in displacement tasks. First, there was a marked tendency to respond in certain ways that subjects had to inhibit to achieve a successful search. Thus, all species had serious difficulties with non-adjacent trials. Thus, upon choosing one of the containers forming a straight line, subjects experienced great difficulty if they had to skip the next empty container. This error was observed independently of whether there was a displacement of the reward involved because it appeared both in the inhibition task (no displacement) and the object permanence task. Call (2001) suggested that this resulted from an inhibition problem. Subjects could not inhibit the tendency of choosing the closest container to their hand. In contrast, de Blois et al. $(1998,1999)$ argued that errors in the nonadjacent trials resulted from a memory problem. Subjects experienced difficulties recalling the exact location where the second item may have been. However, Call (2001) argued that memory alone was unlikely to explain the results because subjects faced the same number of containers and rewards both in adjacent and nonadjacent trials. The only thing that changed was the location of the objects. Interestingly, children, who as a group experienced this difficulty as much as the apes, showed a tendency to select the middle cup first choice in the non-adjacent conditions even though they had seen it empty a few seconds earlier. Call (2001) 
postulated that a possible explanation for this result was the equally strong influenced that the two containers, and not that they had forgotten about the location of the food. In fact, it is precisely because they remembered the location of the rewards in this setup that they experienced difficulties.

Recently, Beran al al (2005) contrasted the inhibition and the memory hypothesis by investigating chimpanzees' accuracy in retrieving two rewards each placed under orte of 5- or 7-container arrays (depending on the experiment) forming a straight line. Subjects were highly accurate in their first choice regardless of its position in the array but performance deteriorated in their second choice as a function of the location of the second reward in relation to the reward that subjects had just recovered. Subjects produced their lowest performance when there was a single empty container between the two baited ones but steadily recovered proportionally to the distance between the two baited cups. Thus, subjects performed better in those trials in which there were 5 compared to 2 empty containers between the two baited containers. Subjects also performed at high levels when the two baited containers were adjacent (i.e., there was no container between them). Beran et al. (2005) convincingly argued that a memory failure rather than a lack of inhibitory control was a more likely explanation for the errors observed. Otherwise, one may have expected to see a distribution of errors independent from the distance between the baited containers (and this was not the case).

However, if a memory failure was solely responsible for these data one would expect that search errors would be equally distributed around the vicinity of the remaining baited container. Of particular interest, are those trials in which the containers $\mathrm{A}$ and $\mathrm{C}$ were baited while the container $\mathrm{B}$, located in between $\mathrm{A}$ and $\mathrm{C}$ was empty. The inhibition hypothesis predicts that errors would be concentrated on container $\mathrm{B}$ whereas the memory hypothesis predicts that errors would be equally distributed to the left and to the right of container $C$. Beran et al. (2005) reported that subjects incorrectly searched container B in 48 trials compared to 31 trials for the alternative (pooling the data across subjects and experiments), Chi-square = $3.66, \mathrm{df}=1, p=0.056$. Although not significant, these data come tantalizingly close to supporting the inhibition hypothesis.

A further analysis involving only those trials in which containers $A$ and $C$ do not occupy the first or the last position in the row of containers seems appropriate. Using those 'central' trials one could analyze the distribution of responses to three locations: left of container $A$, right of container $A$ (which is the same as left of container $C$ ) and right to container $C$. The inhibition hypothesis would predict that the center position would be more likely than either of the side positions to the left and to the right of $\mathrm{A}$ and $\mathrm{C}$, respectively. Thus, while some of the data suggest that a memory failure is a more likely explanation for the observed errors, other data support the inhibition hypothesis.

Second, as noted above, there are also clear indications that memory load (and certain object-container configurations) determine successful object searches. 
Clearly the most obvious indication of the role of memory is the substantially inferior performance in delayed trials compared to non-delayed trials in the delayed response task. In addition, a comparison of the three types of displacement tasks also suggests that the ability to keep track of multiple elements in the task plays an important role. Recall that apes and children performed best in the object permanence task and worse in the rotation task. This pattern of results could be a consequence of the number of elements that changed position during a displacement. In rotations all three elements (platform, containers, and reward) experimented location changes whereas in object permanence displacements only the reward changed location. Also note that children had more difficulty in those displacements within each task in which the reward changed location. For instance, children found $180^{\circ}$ rotations with reward change harder than $180^{\circ}$ rotations without reward change.

Transpositions are a test case for the hypothesis that success is directly dependent on the number of elements that change locations. Since there are only two elements that experimented locations changes, the reward and the containers (but not the platform) one would predict that the difficulty level of this task would fall between that of the object permanence and the rotation displacements. Results indicated that apes found transpositions comparable to object permanence displacements and both tasks easier than rotations. In contrast, children found transpositions comparable to rotations and both tasks harder than object permanence displacements. Indeed, the difference between apes and children in transpositions is striking. Only 4 of 24 children solved this problem compared to 17 of the 24 apes. This result is quite puzzling, although, not unexpected, as previous reports suggested that transposition problems are harder for children to solve than object permanence tasks (Sophian \& Sage, 1983). Additional studies showed that children younger than five years of age have trouble with spatial transpositions (Sophian, 1984, 1986).

So why might transpositions be so difficult for children? According to Piaget (1954) it is not until after the sensorimotor stage of development that children understand the movements of hidden objects, because they do not understand the representation of the cup as containing the object. One possibility is that children did not track the displacement of objects when the container also changed locations. This would explain why they succeeded in those transpositions and rotations that did not involve a change of the reward initial location (i.e., reverse swap, unbaited swap, $180^{\circ}$ in center, and $360^{\circ}$ ) but failed in those where the reward changed locations (single and double transpositions and $180^{\circ}$ side rotation). Some evidence suggests, however, that infants before the representational stage of development understand that objects hidden under a cup move with the cup (Sophian, 1985). The children in our study were old enough to understand that the object moved with the cups. We suggest that the challenges of the transposition task are the sequences of movements in which relevant containers are easily confused with irrelevant containers. That is, the children do not only have to represent the 
container as containing the object but have to update information constantly especially in double transpositions. In contrast, apes found transpositions comparable to object permanence tasks in the current study and some studies have even indicated that transpositions are easier for apes than object permanence displacements (Beran \& Minahan, 2000; Call, 2001).

Although the results on transpositions do not perfectly fit the hypothesis of an inverse relation between performance and the number of elements that change location in the displacement, it is still possible to argue that apes and children have different thresholds for the number of elements that they can track during displacement tasks. Thus, 2.5-year-old children can cope with transformations in one element whereas apes can cope with transformation of up to 2 elements. The alternative to this hypothesis is that some types of displacements are intrinsically harder than others regardless of the number of elements that undergo spatial changes. For instance, apes found $180^{\circ}$ rotations in which the reward did not change locations (because it was under the center container) significantly harder than transpositions in which the reward changed location even though in both tasks two elements changed their initial locations. Conversely, children found that kind of rotation easier than that kind of transposition.

Thus, there may be at least two factors related to attention and memory that influence apes' performance in displacement tasks. One is the number of elements involved in the transformation and the other is the type of transformation taking place. The more elements change location the harder the task becomes for both apes and children. Moreover, 2.5-year-old children find those tasks in which the containers change locations particularly hard whereas apes find hard those tasks in which the platform on which three or more containers rest particularly difficult. Note that apes perform well on various rotations with two, three or five containers (Beran et al., 2005; Call; 2003).

We can also draw some implications from the species differences that we detected. Focusing on apes, chimpanzees and bonobos perform better on spatial transpositions than gorillas and orangutans. Motivation alone cannot explain our results because members of the genus Pan were not different from gorillas or orangutans in certain tasks. Besides, if motivation alone could explain species differences, we would expect to find more consistent inter-species differences across tasks. It is also not the case that the particular chimpanzees that we tested were simply smarter than the later because other studies conducted on the same group of individuals have shown no substantial differences between species or have in fact produced the opposite pattern of results to the current study (Call, 2004; Suda \& Call, 2004). For instance, Suda and Call (2004) found that orangutans outperformed chimpanzees and bonobos in Piagetian conservation tasks. This suggests that interspecies differences are task dependent and points to the wholly unsurprising (but often ignored) conclusion that ape cognition is not monolithic but it is open to specialization in each species. One important task for the future will be to map where the similarities and the differences across species reside. 
Another important issue is the comparison between apes and other species. The data available suggests that apes differ from cats and dogs in displacement tasks (Collier-Baker, Davis \& Suddendorf, 2004; Doré et a]., 1996). Doré et al (1996) reported that cats and dogs failed transposition problems comparable to those that apes solved in the current and previous studies (e.g., Beran \& Minahan, 2000; Call, 2003). Likewise, Collier-Baker et al. (2004) found that dogs solved invisible displacements using the proximity to certain items compared to apes that used representational strategies.

Parrots and corvids perform better than cats and dogs and appear capable of solving various types of invisible displacements (Pepperberg, 1986; Pepperberg \& Funk, 1990; Pollok, Prior \& Gunturkun, 2000). However, these results are not uncontroversial because they are based on the Uzgiris-Hunt scale to measure object permanence. Several authors have criticized this scale because correct responses are scored according to the order of searches rather than on whether subjects search only in possible places (e.g., Dore et al., 1996; Sophian, 1985). For instance, in the last (and presumably most demanding) item of this scale (i.e., three consecutive invisible displacements), subjects are credited with passing this item if they search under the last cover visited by the experimenter or they search in the reverse order to the trajectory described by the object. However, it is unclear that searching under the first cover first is incorrect. If the subject has a representational notion of objects, they could be under any of the covers and the search order is irrelevant. Searching all covers irrespective of order should be acceptable. But this raises another problem. If one disregards the search sequence, subjects could solve the problem using a non-representational strategy consisting of searching under all locations until finding the object. The solution to this second problem, which a number of researchers have adopted, is to displace the object only under two of the three available containers (Sophian, 1985; Natale et al., 1986) and scoring searches to the non-visited container as incorrect. Recently, Pepperberg et al. (1997) found that two African grey parrots not only solved this kind of displacement but also a transposition displacement.

There is also some evidence that apes outperform monkeys in invisible displacement tasks (e.g., de Blois et al., 1998). However, caution is needed in establishing broad comparisons across taxa because only a handful of monkey species have been investigated under controlled situations. There are indeed some studies that found no differences between monkeys and apes in several invisible displacements such as single displacements (de Blois \& Novak, 1994; Neiworth, Steinmark, Basile, Wonders, Steely and DeHart, 2003). Neiworth et al. (2003) even found that cotton-top tamarins solved double invisible displacements above chance. However, their methodology was based on allowing subjects to choose only one of the containers. Since subjects showed a marked tendency to touch the last container visited by the experimenter, one may interpreted this in terms of a recency effect (see also Beran et al., 2005), which as the authors indicated did not appear in visible displacements. Allowing subjects to select two containers and varying the final 
location of the reward between the first and second container visited may have helped to disambiguate this result. It is therefore yet unclear whether apes and monkeys differ substantially in object permanence or any other type of displacement, for which there is little data availlable to compare species.

In conclusion, this series of object displacement tasks proved to be a sensitive and easily applicable tool for measuring individual and species differences among great apes and young children. We confirmed many similarities and uncovered some striking differences among apes including children in object displacement tasks. One of the most promising avenues for future research is the use of batteries of tasks like this as standardized tests across several labs. Results from different populations could then be compared and would provide interesting insights into variables such as rearing history and sex differences that are usually unaddressed in comparative studies due to low number of subjects.

\section{ACKNOWLEDGEMENTS}

We thank Jana Jurkat, Angela Loose, Eva Leerman, Katharina Haberl, and several student assistants for thelp in the organization of this study. We are very grateful to the children, parents, and Kindergarten staff for their cooperation and participation in this study. We also thank the keepers at the Wolfgang Köhler Primate Research Center for their help and support. 


\section{REFERENCES}

Baillargeon, R. (1987). Object permanence in 3.5- and 4.5-month-old intants. Developmental Psycholog, 23, 655-664.

Beran, M., \& Minalian, M. (2000). Monitoring spatial transpositions by bonobos (Pon poniscus) and chimpanzees (Pan troglodytes). International Journal of Comparative Psychology, 13, 1-15.

Beran, M. J., Beran, M. M., \& Menzel, C. R. (2005). Spatial memory and monitoting of hidden itens through spatial displacements by chimpanzees (Pan troglodytex). Journal of Comparative Psychology, 119, 14-22.

Butterworth, G. E. (1977). Object disappearance and error in Piaget's stage IV task. Journal of Experimental Child Psychology, 23, 162-173.

Call, J. (2000). Representing space and objects in monkeys and apes. Cognithe Schence, 24, 397-422.

Call, J. (2001). Object permanence in orangutans (Pongo plgandeus), chimpanzees (Pan troglodytes), and children (Homo sapiens). Joumal of Compararive Psycholog, $115,159-1,71$.

Call, J. (2003). Spatial rotations and transpositions in orangutans ( $P_{\text {ong }}$ pygmieus) and chimpanzees (Pan troglodytes). Primates, 44, 347-357.

Call, J. (2004). Inferences about the location of food in the Great Apes (Pan paniscus, Pan troglodutes. Gorilla gorilla, and Pongo pygmaeus). Journat of Comparative Psychology, 118, 232-241.

Collier-Baker, E., Davis, J., \& Suddendorf, T. (2004). Do dogs (Canis familiaris) understand invisible displacement? Joumal of Comparative Psychology, 118, 42.1-433.

de Blois, S. and Novak, M.A. (1994). Object permanence in thesus monkeys (Macaca mrulatta). Joumal of Comparative Psychology, 108, 318-327.

de Blois, S.T., Novak, M.A. \& Bond, M. (1998). Object permanence in orangutans (Pongo pygmceus) and squirrel monkeys (Saimiri sciureus). Joumal of Compararive Psychology, $112,137-152$.

de Blois, S.T. \& Novak, M.A. \& Bond, M. (1999). Can memory requirements account for species differences in invisible displacenent tasks. Joumal of Experimental Psychology: Animat Behovior Processes, 25, 168-176.

Doré, F. Y., \& Dumas, C. (1987). Psychology of animal cognition: Piagetian studies. Psychological Bulletin, 102, 219-233.

Dore, F.Y., Fiset, S., Goulet, S., Dumas, M.C. \& Gagnon, S. (1996). Search behavior in cats and dogs: Interspecific differences in working memory and spatial cognition. Animal Learning of Behovior, 24, 142-149.

Diamond, A. (1985). Development of the ability to use recall to guide action, as indicated by infants' performance on $A B$. Child Development, $56,868-883$.

Harlow, H. F. (1932). Comparative behavior of primates. III. Complicated delayed reaction tests on primates. Joumal of Comparative Psychology, 14, $241-252$.

Hunter, W.S. 1913. The delayed reaction in animals and children. Behavior Monographs, 2. 1+86.

Lasky R. E., Romano N., Wenters J. (1980). Spatial localization in children after changes in position. Jounal of Experimenal Child Psychology, 29, 225-248.

Mendes, N. \& Huber, L. (2004). Object permanence in common marmosets (Callithrix facchus). Journal of Comparative Psychology, 118, 103-112.

Natale, F., Antinucci, F., Spinozzi, G., \& Poti, P. (1986). Stage 6 object concept in nonhuman primate cognition: A comparison between gorilla (Gorilla gorilla gorilla) and Japanese macaque (Macaca fuscata). Journal of Comparative Psycholagy, 100, 335-339.

Neiworth, J.J., Steinmark, E., Basile, B.M., Wonders, R., Steely, F. \& DeHart, C. (2003). A rest of object permanence in a New World monkey species, cotton top tamarins (Saguimus oedipus). Animal Cognition, 6, 27-37.

Pepperberg, I.M. \& Funk, M.S. (1990). Object permanence in four species of psittacine birds: An African grey parrot (Psiffacus erithacus), an Illiger mimi macaw (Ara maracana), a parakeet (Melopsitracus undulatus), and a cockatiel (Nymphicus hollandicus). Animal Learning \& Behavior, $18,97-108$.

Pepperberg, I.M. \& Kozak, F.A. (1986). Object permanence in the African grey parrot (Psiflacus erithecus). Animal Learning \& Behavior, 14,322-330.

Pepperberg, LM., Willner, M.R. \& Gravitz, L.B. (1997). Development of Piagetian object permanence 
in a Grey parrot (Psittacus erithacus). Jownd of Comparative Psychology, 111, 63-75.

Piagel, J. (1954). The construction of reality w the child. NV: Basic Books.

Pollok. B., Prior, H. \& Guentuerkuen, O. (2000). Dewelopment of object permanence in food-storing magpies (Pica pica). Journal of Comparative Psycholog, $114,148-157$.

Poti. P. (2000) Aspects of spatial cognition in capuchins (Cebus apello), frames of reference and scale of space Animal Cognition, 3,69-77.

Sophian, C. (984). Spatial transpositions and the early development of search. Developmental Psychology, 20,21-28.

Sophian, C. (1985) Undenstanding the movements of objects: Early developments in spatial cognition. Brittih Jownal of Developmental Psychology, 3, 321-333.

Sophian, C, (1986). Early developments in children's spatial monitoring Cognition, 22, 61-88.

Sophian, $C_{*}$ \& Sage, S. (1983). Developments in infants' search for displaced objects. Joumal of Experimental Child Pychology, 35, 143-160.

Suda, C, \& Call, J. (2004). Piagetian liquid conservation in the great apes (Par paniscus, Pat troglodytes, and Pongo pygmaeus). Joumal of Comparative Psychology, 118, $265-79$.

Tomasello, M. \& Call, J. (1997). Primate Cognition. Ox ford University Press: New York.

Visalberghi, E. (1986). Aspects of space representation in an infant gorilla. In D.M. Taub \& F.A. King (Eds.). Current Perspectives in Primate Social Dynamics (pp. 445-452). New York: Van Nostrand Reinhold Company. 


\section{II}

SEARCHING FOR HIDDEN

OBJECTS IN THE PRESENCE OF SOCIAL CUES 


\section{CHAPTER 2}

Limitations in the spontaneous use of referential cues by great apes and young children 


\section{Chapter 2}

\section{ABSTRACT}

The ability of great apes $(n=17)$ and $2 \frac{1}{2}$-year-old children $(n=24)$ to spontaneously use the directional reference of social cues to locate hidden objects was investigated. Unlike previous studies, the subjects were dependent solely on social cues and did not receive any training with non-social cues. Moreover, in contrast to previous studies the cues were not administered with an increasing level of difficulty but in a randomized design. As a group, neither the apes nor the children performed above chance levels at any of the cues. However, in contrast to the apes some children showed results significantly above chance. The limited use of social cues reported in this study is discussed in the context of the variety of experimental designs that have been used to study the use of social cues with object-choice tasks. 


\section{INTRODUCTION}

For individuals of social groups, the interpretation of social cues, such as head and eye orientation of other individuals, has a potentially adaptive value, as they can provide directional cueing to interesting locations, objects, or behawiors (Chance, 1967; Fehr \& Exline, 1987). The sensitivity to social cues can promote the exchange of information that is possessed by some individuals but not by others. Already shortly after birth human as well as chimpanzee infants are sensitive to the face and eye-direction of their caregivers (e.g. Hood, Willen, \& Driver, 1998; Farroni, Csibra, Simion, \& Johnson, 2002; Farroni, Massaccesi, Pividori, \& Johnson, 2004; Myowa-Yamakoshi, Tomonaga, Tanaka, \& Matsuzawa, 2003, Okamoto et al. 2002; Okamoto, Tanaka, \& Tomonaga, 2004).

Gaze-following, looking where somebody else is looking (Butterworth \& Jarrett, 1991; Corkum \& Moore, 1995) is a mechanism that can facilitate the response to social cues as it may direct the attention of another individual to the object of interest. A wealth of studies has documented gaze-following as a reliable behavior in response to social cues in young children (e.g. Scaife and Brunner, 1975; Butterworth and Cochran, 1980; Butterworth and Jarrett, 1991) and nonhuman primates (e.g. Itakura, 1996; Kaplan and Rogers, 2002; Lorincz, Baker, \& Perret, 1999; Povinelli \& Eddy, 1996a, 1996b, 1997; Povinelli, Dunphy-Lelii, Reaux, \& Mazza, 2002; Tomasello, Call, \& Hare, 1998; Tomasello, Hare, \& Agnetta, 1999; Tomasello, Hare, and Fogleman, 2001).

It is however less clear, how well nonhuman primates and young children understand directional social cues in intentionally communicative contexts. One way of finding out how well nonhuman primates and children are able to extract information from nonverbal cues is with the object-choice paradigm. In this paradigm an experimenter hides an object under one of two opaque cups and then gives a cue such as gazing or pointing to the location were the object has been hidden. The subject then is allowed to make a choice between two locations. This paradigm has been mostly used to study the understanding of interaction in nonhuman primates (e.g. Anderson, Sallaberry, \& Barbier, 1995; Anderson, Montant, \& Schmitt, 1996; Barth, Reaux, \& Povinelli, 2005; Byrnit, 2004; Call, Agnetta, \& Tomasello, 2000; Call, Hare, \& Tomasello, 1998; Call \& Tomasello, 1994; Hare \& Tomasello, 2004; Itakura \& Anderson, 1996), but a few studies also additionally included children as subjects (Itakura \& Tanaka, 1998; Povinelli, Reaux, Bierschwale, Allain, \& Simon, 1997; Povinelli, Bierschwale, \& Cech, 1999; Tomasello, Call, \& Gluckman, 1997). Other animals like dogs (e.g. Agnetta, Hare, \& Tomasello, 2000; Hare, Brown, Williamson, \& Tomasello, 2002; Hare \& Tomasello, 1999; Soproni, Miklosi, Topal, \& Csanyi, 2001; Miklosi, Polgardi, Topal, \& Csanyi, 1998; McKinley \& Sambrook, 2000), seals (Shapiro, Janik, \& Slater, 2003; Scheumann \& Call, 2004), and horses (McKinley \& Sambrook, 2000) have also been tested with this paradigm.

Despite obvious reasons to assume that gaze-following would be the essential mechanism that is sufficient to succeed in such object-choice tasks, the results show a different picture. There seems to be a double paradox in the 
discrepancy between the ability for gaze-following and the use of social cues in object-choice tasks (Call, 2004). On the one hand, apes and monkeys are very reliable followers of a human and conspecific gaze (e.g Tomasello, Call, \& Hare, 1998; Tomasello, Hare, \& Agnetta, 1999) but seem to have a rather limited ability of exploiting the communicative intent in such gaze cues as it is operationalized in the object-choice task. Dogs on the other hand, do not seem to follow the gaze of humanis (Agnetta, Hare, \& Tomasello, 2000), but do follow their directional cues to locate hidden food more proficiently than chimpanzees or wolves, which is probably a domestication effect (Hare, Brown, Williamson, \& Tomasello, 2002). It seems that gaze-following is rather a componential but not a sufficient mechanism to successfully use social cues to locate hidden objects. Other features (not related to gaze-following) of the provided cues may play an additional role (e.g. proximity, novement).

However, the original rationale of the object-choice task involving social cues was the investigation of the ability to understand the direction of looking as referential communication (e.g. Anderson, Sallaberry, \& Barbier, 1995). The results from cues that are facilitated by their physical properties (e.g. touching, proximate pointing) are complicated by a classic phenomenon in animal behavior: local enhancement (Thorpe, 1956). Cues that can be used based on their physical properties do not necessarily prove an understanding of the referential communicative intent of such cues. For example, chimpanzees seem to be able to use a proximate pointing cue but not a distant pointing cue in contrast to 3-year-old children's understanding of both cues (Povinelli et al. 1997). Moreover, several previous studies have used a step-wise design in which the experimental cues are provided with an increasing level of difficulty. Subjects are then only advanced to the next level of difficulty if they have mastered the easier cue. It has been shown that by applying this method nonhuman primates may eventually learn some cues that they would presumably not have learned otherwise (e.g Itakura \& Anderson, 1996; Vick \& Anderson, 2000).

Therefore, I was interested to which extend great apes and young children are able to exploit social cues if they do not receive any exposure to physical cues prior to social cues and if they do not receive the cues in a step-wise design. I administered the experimental trials in a randomized design and administered only cues of a social and referential nature.

So far no study with children (and apes) has been conducted in which the interaction between the experimenter and the subject was recorded and analyzed. This analysis seems crucial because it lies at the very heart of the rationale of this experimental paradigm. Only if it can be shown that a correct choice is immediately preceded by a following response to the experimenters cue, we will know that the communicative aspect of the task facilitated the response. Therefore, in the present study I recorded the interaction between the experimenter and the child in a merged picture to investigate the relation between the subjects' responses to the cues and 
the rate of success in finding the hidden toys'. Additionally, 1 recorded and analyzed the children's manual responses to the cue (e.g. reaching or pointing). Moreover, to the best of our knowledge, no previous studies explicitly paid attention to the confounding influence of perseveration errors. In the present study, I compared the subjects choices with the location of the object on the previous trial to analyze whether the subjects showed any bias to search in the location they had previously seen the object.

\section{EXPERIMENT 1 - APES}

In the first experiment 17 apes were tested for their understanding of the communicative intent of social cues. All apes had never been tested with this paradigm before and I was interested whether subjects naive to this task would spontaneously use any of the cues to locate hidden food.

\section{Subjects}

\section{METHOD}

Ten chimpanzees (Pan troglodytes), four bonobos (Pan paniscus), and three gorillas (Gorilla gorilla) participated in this study. All seventeen apes were housed in social groups with their conspecifics at the Wolfgang Köhler Primate Research Center located at the Zoo Leipzig, Germany. They were fed three times a day with their species typical diets of vegetables and fruit. Water was available ad libidum. Subjects were never deprived of food and water at any time. All subjects were used to being tested in specially designed observation rooms. For an overview of the subjects' sexes, ages, and rearing histories see Table 1.

\section{Apparatus}

A little opaque white cup $(5,5 \mathrm{~cm} \times 6 \mathrm{~cm})$ was used to contain the food rewards (slices of banana, grapes, or juice). This cup will be referred to as the 'food-cup'. Two larger opaque white cups $(7,5 \mathrm{~cm}$ high and $9 \mathrm{~cm}$ in diameter) were used to conceal the food-cup. A square blue colored bowl was used to conceal the hiding process on experimental trials. A wooden platform $(82 \mathrm{~cm} \times 30 \mathrm{~cm})$ was attached to the metal frame of the Plexiglas observation window inside the testingunit. A panel $(82 \mathrm{~cm} \times 30 \mathrm{~cm})$ rested on the platform and could be slid back and forward to present the task to the subjects. The cups were positioned on this

\footnotetext{
'This interaction was only analyzed for the children in Experiment 2, because the experimental set-up made it impossible to collect comparable data on this wariable for the apes in Experiment 1 .
} 
platform $65 \mathrm{~cm}$ apart from each other ${ }^{2}$. A little piece of wood was glued to the panel at the center of the outer side that was closest to the experimenter. The experimenter used this handle to slide the panel back and forth. At the outer corners of the panel two little barriers were glued on to enable a correct positioning of the cups.

TABLE 1 Age, Sex, and Rearing History for all subjects in Experiment 1.

\begin{tabular}{llccc}
\hline & Species & Age & Sex & Rearing \\
\hline Name & Chimpanzee & 25 & $\mathrm{~F}$ & nursery \\
\hline Fraukje & Chimpanzee & 9 & $\mathrm{~F}$ & mother \\
Jahaga & Chimpanzee & 8 & $\mathrm{~F}$ & mother \\
Gertruidat & Chimpanzee & 4 & $\mathrm{M}$ & mother \\
Patrick & Chimpanzee & 24 & $\mathrm{~F}$ & nursery \\
Ulla & Chimpanzee & 8 & $\mathrm{~F}$ & mother \\
Fifi & Chimpanzee & 24 & $\mathrm{~F}$ & nursery \\
Riet & Chimpanzee & 8 & $\mathrm{~F}$ & mother \\
Sandra & Chimpanzee & 21 & $\mathrm{~F}$ & nursery \\
Natascha & Chimpanzee & 8 & $\mathrm{M}$ & mother \\
Frodo & Bonobo & 8 & $\mathrm{~F}$ & mother \\
Uitindi & Bonobo & 19 & $\mathrm{M}$ & nursery \\
Joey & Bonobo & 6 & $\mathrm{M}$ & nursery \\
Limbuko & Bonobo & 5 & $\mathrm{M}$ & nursery \\
Kuno & Gorilla & 6 & $\mathrm{M}$ & mother \\
Vimoto & Gorila & 6 & $\mathrm{~F}$ & mother \\
Viringika & Gorilla & 6 & $\mathrm{~F}$ & mother \\
Vizuri & & & & \\
\hline
\end{tabular}

\section{Procedure}

The experimenter sat behind the table in front of the subject. The subject sat behind the Plexiglas partition with three little openings that allowed the subjects to insert a finger and make a choice by touching one of the cups on the table (Figure 1). From their training in research procedures all subjects were very familiar with this interaction. The subjects received an informal orientation session in which the experimenter presented the three cups to the subject. The experimenter put fruit or juice in the food-cup and showed it to the subject. Then he put the food-cup into the middle of the panel and placed the two larger cups next to it with the food-cup being in the middle. The experimenter than took one of the cups with his right hand and placed it over the food-cup. Again, with his right hand he took the other cup

\footnotetext{
2Prior to this study 1 conducted an informal pilot study with six orangutans. In this study we mamipulated the distance between the cups so that they were either $65 \mathrm{~cm}$ or $120 \mathrm{~cm}$ apart. The orangutans' responses to the same cues as reported here were similar to the results of the apes in the present study independent of the distance between the cups.
} 
and simply placed it on the panel making the same movement and sound as with the first cup. He then put his left hand on the left cup and his right hand on the right cup and slid the cups into the outer positions. The experimenter then looked straight ahead with a neutral face with his eyes looking above the subject without making any eye-contact. The experimenter waited for 10 seconds and then slid the panel against the Plexiglas window so that the cups were positioned in front of the two outer openings in the Plexiglas. The first cup the subjects touched was turned over by the experimenter. If it contained the food-cup the subject received its contents.

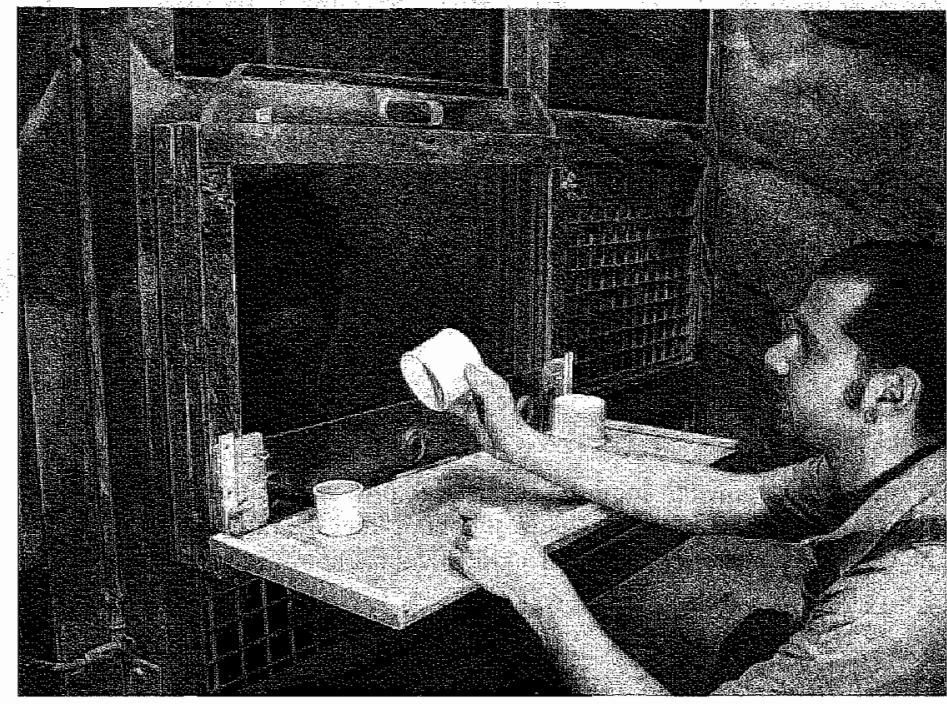

FIGURE 1. The experimental set-up in Experiment 1. After the subject (Fifi) touched one of the cups the experimenter lifts the cup under which the food-cup had been placed.

If the subject selected the cup that did not contain the food-cup they were simply shown that it was empty. The panel was slid back again and a new trial was started. Subjects had to find the food-cup on five of six trials. Since the subjects were already familiar with this procedure and interaction with the experimenter, all subjects recovered the food on at least five trials within the first session. The experiment was started on the next available day after the subject passed the orientation. The experimental trials were administered in a similar fashion as the orientation trials with the modification that the experimenter now used the square plastic bowl to conceal the covering of the food-cup. For each trial after the cups were positioned in the middle of the panel the experimenter took the bowl with his left hand and held it over the cups. He then reached under it with his right hand and covered the food-cup like during the orientation trials. He then put the bowl on the 
floor again and proceeded with sliding the cups into their outer positions. As soon as the cups were in position the experimenter called the subject's name. As soon as the experimenter established eye-contact with the subject he gave one of the following three cues to the cup that contained the food: gaze, glance, or point (see Figure 2). Subjects received 48 trials in total distributed over 8 sessions of 6 trials. Within a session each of the three cues were given once to the left and once to the right cup. The food was never hidden under the same cup more than two times in a row. The location of the food and the type of cue was randomized exhaustively for each session. Subjects typically received only one session a day and were typically tested on subsequent days. Subjects typically completed the experiment in about 10 days.
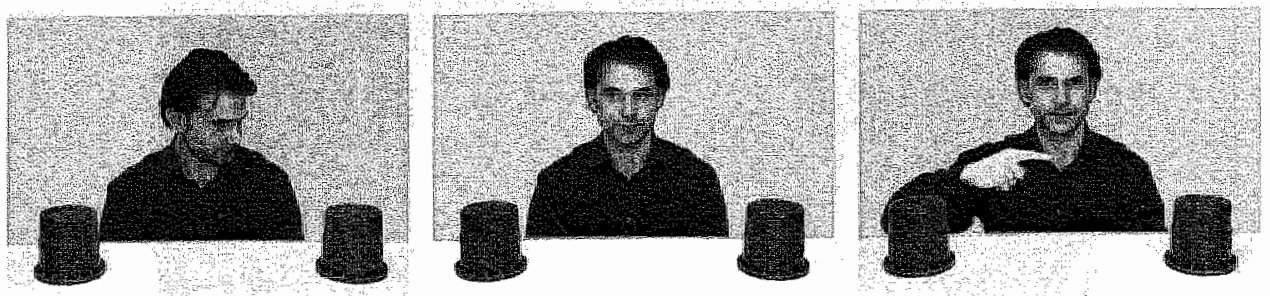

FIGUIRE 2 Photographs showing the three social cues provided to the subjects. From left to right: Gaze, glance, point. Note. The pictures show the cups and platform used with the children in Experiment 2. However, the cues were given in an identical fishion for the apes and the children.

\section{Data scoring and inter-observer reliability assessment}

All trials were scored online by the experimenter. A second rater coded 50 percent of the trials of each subject from video recordings. The inter-observer agreement was calculated by using Cohen's Kappa. The video rater was requested to code which cup the subjects touched first after the 10-seconds delay. The video scoring matched the records of the experimenter on almost a hundred percent of the trials (407/408) resulting in a Kappa value of $k=.995$.

\section{RESULTS}

Table 2 shows the mean percentage of correct choices per individual and cue. I used a one sample t-test to compare the apes' performance against chance $(p=50)$. On the overall score the apes $(n=17)$ did not select the correct cup more often than would be expected by chance, $t(16)=0.554 ; p=$ ns. When each of the three cues were considered separately the apes did also not select the correct cup more often than would be expected by chance, gaze cue, $t(16)=0.618 ; p=n$ ns, glance cue, t(16) $=0.334 ; p=n s$, and pointing cue, $t(16)=0.591 ; p=n s$. A one-way ANOVA showed no difference between species for the overall score $F(2,14)=0.466, p=n$, 
the gaze cue $F(2,14)=2.657, p=n$ s, the glance cue $F(2,14)=0.195, p=n$, and the pointing cue $F(2,14)=1.038, p=$ ns.

TABLE 2. Mean percentage of correct trials per subject and cue for Experiment 1. Values represent the mean percentage of correct choices of 16 administered trials per cue.

\begin{tabular}{llcccc}
\hline Name & Species & $\begin{array}{c}\text { Gaze } \\
\mathbf{n = 1 6}\end{array}$ & $\begin{array}{c}\text { Glance } \\
\mathbf{n}=\mathbf{1 6}\end{array}$ & $\begin{array}{c}\text { Point } \\
\mathbf{n}=\mathbf{1 6}\end{array}$ & $\begin{array}{c}\text { Totall } \\
\mathbf{n}=\mathbf{4 8}\end{array}$ \\
\hline Fraukje & Chimpanzee & 44 & 44 & 50 & 46 \\
Jahaga & Chimpanzee & 25 & 38 & 44 & 35 \\
Gertruida & Chimpanzee & 31 & 50 & 38 & 40 \\
Patrick & Chimpanzee & 50 & 56 & 44 & 50 \\
Ulla & Chimpanzee & 63 & 56 & 75 & 65 \\
Fifi & Chimpanzee & 63 & 50 & 44 & 52 \\
Riet & Chimpanzee & 50 & 50 & 44 & 48 \\
Sandra & Chimpanzee & 38 & 69 & 38 & 48 \\
Natascha & Chimpanzee & 44 & 56 & 44 & 48 \\
Frodo & Chimpanzee & 44 & 50 & 50 & 48 \\
Ulindi & Bonobo & 44 & 57 & 44 & 48 \\
Joey & Bonobo & 63 & 63 & 44 & 56 \\
Kuno & Bonobo & 69 & 31 & 44 & 48 \\
Vimoto & Gorilla & 50 & 50 & 44 & 48 \\
Viringika & Gorilla & 44 & 44 & 56 & 48 \\
Vizuri & Gorilla & 38 & 56 & 69 & 54 \\
Mean \% & & & 58 & 49 & 49 \\
\hline
\end{tabular}

To compare the results of the apes with the results of the children, who received 12 instead of 48 trials, I used a binomial test to analyze the apes" individual overall scores for the first 12 trials. None of the subjects selected the correct cup more often than would be expected by chance. The highest score was 9 out of 12 trials correct (Joey). Further, to analyze the subjects' perseveration errors, I compared the subjects' choices with the location of the food on the previous trial ${ }^{3}$. Subjects chose the cup under which they had seen the food on the previous trial on $55 \%$ of the trials (372/680). Additional individual analyses by using the binomial test showed no significant differences between the subjects' choices. Only Fraukje showed a preference, but she chose the cup under which the food had not hidden on the previous trial significantly more often than the other cup $(p=0.038$, binomial test). When I compared the actual location of the food on the previous trial with the location on the following trial, remember that the food was never in the same

\footnotetext{
${ }^{3}$ Since the subjects received 6 trials in a session per day, the first trial was excluded from the analysis. Thus, the measure is based on 40 trials of the 48 trials that each subject received.
} 
location more than two times in a row, I found that there was a higher probability that the food was in a different location than on the previous trial ( 490 versus 190). Moreover, when I analyzed the subjects' side bias per individual by using a binomial test I found that 8 out of 17 subjects had significantly more correct choices on one side than the other.

\section{DISCUSSION}

In Experiment $1 \mathrm{I}$ investigated the ability of great apes to spontaneously use social cues that were provided by an experimenter to find food hidden under one of two cups. None of the apes selected the correct cup more often that would be expected by chance. There were no differences between species and no differences between the three cues. Additionally, I found that the subjects showed a bias to choose the location were they had seen the food on the previous triall. Also, I found that about half of the subjects preferred one side over the other. The results of Experiment 1 show clearly that, under the given conditions (e.g. without prior exposure to physical cues and a step-wise design), the apes were unable to use any of the cues to find the hidden food.

\section{EXPERIMENT 2 - CHILDREN}

In the second experiment I adapted the task given to the apes in Experiment 1 slightly to be suitable for children. All children I tested were like the apes naïve to this paradigm. I was interested whether children, contrary to the apes in Experiment 1 , would spontaneously use any of the social cues to locate hidden toys. The general procedure and the cues were identical to the methods used in Experiment 1.

\section{Subjects}

\section{METHOD}

Participants. Twenty-four children (12 females, 12 males) of 2.5-2.7 years of age (mean $=21 / 2$ ) from Leipzig, a mid-sized town in Germany, participated in this study. The majority of the children come from a German speaking middle class background. They were recruited from a database that is maintained in collaboration with local Kindergartens. The parents had approved their children's participation in written form. All children were tested at the Kindergartens. The parents regularly received information materials about ongoing studies at the child lab.

\section{Apparatus}

A children's table $(59 \mathrm{~cm} \times 50 \mathrm{~cm}, 50 \mathrm{~cm}$ high) and chair $(30 \mathrm{~cm}$ high) were brought to the Kindergarten. A platform $(66 \mathrm{~cm} \times 60 \mathrm{~cm})$ with a theater-like curtain construction was put on the table (see Figure 3 a). A platform $(78 \mathrm{~cm} \times 30 \mathrm{~cm}$ ) 
resting inside the larger platform allowed the experimenter to slide the task back and forth. The cups were positioned on the platform about $65 \mathrm{~cm}$ apart from each other. Small barriers glued on the outer corners aided the correct positioned of the cups. Small toys were used as the objects the children had to find.
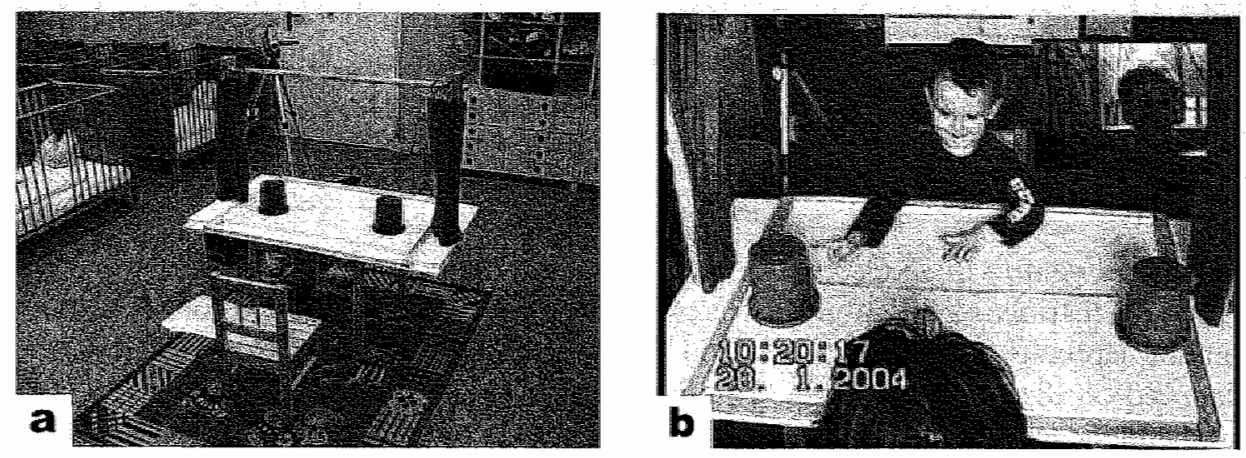

FIGURE 3. (a) The experimental setting in Experiment 2 at the kindergarten. The child is seated in the little chair and facing the experimenter who is sitting at the other side of the table. (b) A still picture of the actual wideo recordings showing the merged picture captured by two video cameras. "The upper little picture shows the cue given by the experimenter (gaze). A young boy points to the cup immediately after the experimenter started gazing at it.

\section{Procedure}

The subjects were seated at the table facing the experimenter. The experimenter sat at the opposite side of the table on the floor to be at eye level with the subject. The subjects first received a number of orientation trials as introduction to the task. The experimenter randomly selected one of the toys from the box and showed it to the subject. He told the subject "Let's see where $X$ (the toy) will be hiding!" He then placed it on the middle of the sliding panel and placed the two cups next to it with the toy being in the middle. He then placed one of the cups over the toy. He made an identical movement and sound with the other cup. He then slid the cups simultaneously to the outer positions on the panel. The experimenter then waited for approximately 10 seconds and said to the child: "Do you still know where it is? Have a look!". He then slid the panel towards the children so they could choose one of the cups and search for the toy. If the child chose the correct cup they played with the toy briefly and then were asked to hand it back to the experimenter who put it aside. If they selected the incorrect cup the panel was pulled back and a new trial was started. After the child had recovered the toy for three trials in a row the experimental trials were started. The experimenter started the trial as during the orientation session, but now closed the curtains before hiding the toy. Once the toy was hidden and the cups were in their outer positions the experimenter opened the curtains again and called the subject's name. As soon as the experimenter established eye-contact with the child he gave one of three social cues to the cup 
that contained the toy in a similar fashion as in Experiment 1 (see Figure 2). Each cue was given for 10 seconds, then the experimenter looked straight ahead and pushed the panel forward to let the subject choose. If the children selected the correct cup they received verbal praise, played with the toy briefly, and a new trial was started. If they chose the incorrect cup, the experimenter pulled back the tray and said. Let's do it again!. All subjects received 12 trials (4 trials of each cue) that were administered randomly and exhaustively with the obligation that the toy was never hidden under the same cup more than two times in a row. The location of the last orientation trial and the first experimental trials were counterbalanced for each cue and between the sexes.

\section{Video Coding and Inter-Observer Reliability}

All trials of all subjects were captured by two cameras (Figure 3.b). One camera captured the experimenter and the other the subject $\mathrm{A}$ merged picture was recorded on digital video tapes. The data were coded from the video tapes by two independent raters. The main rater coded all trials of all subjects. A second rater coded all trials of 6 subjects ( 3 boys and 3 girls) that were randomly selected from the sample. The agreement between the raters was calculated by using Cohen's Kappa. Agreement was found to be excellent for all coded variables. Therefore, only the data from the main rater were used in the analysis. Both raters were asked to code the following variables: (a) 'Cup choice" - Which cup does the subject touch first after the presentation of the cue $(L=L$ eft, $R=$ Right $)$ ? Raters agreed on a $100 \%(72 / 72)$ of the trials $(k=1)$. (b) Looking' - Is the subject looking at one of the cups immediately after the experimenter starts presenting the cue? Record only behavior that is clearly a response to the cue and clearly in the direction of one of the cups $(\mathrm{L}=\mathrm{Left}, \mathrm{R}=\mathrm{Right},-=$ no looking). Raters agreed on $89 \%$ (64/72) of the trials $(k=.83)$. (c) 'Reaching/Pointing' - Is the subject reaching to or pointing at one of the cups during the presentation of the cue? Record only behavior that is clearly directed at one of the cups $(t=$ reaching or pointing, - = no reaching or pointing). Raters agreed on $92 \%(66 / 72)$ of the trials $(k=.80)$. No discussion between raters was necessary for the reported Kappa values.

\section{RESULTS}

Table 3 shows the mean percentage of correct choices per individual and cue. I used a one sample t-test to compare the children's performance against chance $(p=50)$. On the overall score the children $(n=24)$ did not select the correct cup more often than would be expected by chance, $t(23)=1.373 ; p=n s$. When each of the three cues were considered separately the children did also not select the correct cup more often than would be expected by chance, gaze cue, $t(23)=1.000 ; p=n s$, glance cue, $\mathrm{t}(23)=1.543 ; p=\mathrm{ns}$, and pointing cue, $\mathrm{t}(23)=1.273 ; p=\mathrm{ns}$. An undependent samples t-test showed no difference between boys and girls for the overall score, $\mathrm{t}(22)=0.519 ; p=$ ns, the gaze cue $\mathrm{t}(22)=0.829 ; p=$ ns, the glance cue, $t(22)=0.506 ; p=n s$, and the pointing cue, $t(22)=0.537 ; p=$ ns. On the 
individual level three subjects (all boys) selected the correct cup significantly more often than would be expected by chance on the total score $(p<0.05$, binomial test). Five subjects selected the correct cup on all pointing trials, one subject selected the correct cup also on all gazing trials, and one subject selected the correct cup also on all glancing trials. All successful subjects were boys.

TABLE 3 Mean percentage of correct trials per subject and cue for Experiment 2. Values represent the mean percentage of correct choices of 4 administered trials per cue.

\begin{tabular}{|c|c|c|c|c|c|}
\hline Subject & Sex & $\begin{array}{l}\text { Gaze } \\
n=4\end{array}$ & $\begin{array}{c}\text { Glance } \\
\mathbf{n}=4\end{array}$ & $\begin{array}{l}\text { Point } \\
n=4\end{array}$ & $\begin{array}{r}\text { Total } \\
n=12\end{array}$ \\
\hline 01 & male & 75 & 100 & 100 & 92 \\
\hline 02 & male & 25 & 50 & 50 & 42 \\
\hline 03 & male & 75 & 75 & 100 & 83 \\
\hline 04 & male & 50 & 50 & 50 & 50 \\
\hline 05 & male & 25 & 50 & 100 & 58 \\
\hline 06 & male & 75 & 75 & 25 & 58 \\
\hline 07 & male & 100 & 50 & 100 & 83 \\
\hline 08 & male & 50 & 25 & 50 & 42 \\
\hline 09 & male & 25 & 25 & 25 & 25 \\
\hline 10 & male & 25 & 75 & 100 & 67 \\
\hline 11 & male & 75 & 50 & 0 & 42 \\
\hline 12 & male & 50 & 25 & 25 & 33 \\
\hline 13 & female & 75 & 50 & 75 & 67 \\
\hline 14 & female & 75 & 75 & 50 & 67 \\
\hline 15 & female & 50 & 75 & 50 & 58 \\
\hline 16 & female & 50 & 50 & 75 & 58 \\
\hline 17 & female & 25 & 75 & 50 & 50 \\
\hline 18 & female & 50 & 50 & 50 & 50 \\
\hline 19 & female & 50 & 75 & 50 & 58 \\
\hline 20 & female & 75 & 25 & 25 & 42 \\
\hline 21 & female & 25 & 50 & 75 & 50 \\
\hline 22 & female & 25 & 50 & 50 & 42 \\
\hline 23 & female & 0 & 75 & 50 & 42 \\
\hline 24 & female & 50 & 50 & 50 & 50 \\
\hline \multicolumn{2}{|c|}{ Mean $\%$} & 48 & 51 & 49 & 49 \\
\hline
\end{tabular}

When 1 considered only the first trial, remember that the first trial was counterbalanced per cue, location, and sex, I found that the children selected the correct cup more often than would be expected by chance $(p=.50), \mathrm{t}(23)=2.769 ; p$ $=0.011$. Eighteen of the 24 children $(75 \%)$ selected the correct cup on the first trial. When I considered the results for each cue separately I found that the children were only above chance in the pointing cue, $t(7)=3.000 ; p=0.020$, but not in the gaze cue, $\mathrm{t}(7)=1.528 ; p=\mathrm{ns}$, and the glance cue, $\mathrm{t}(7)=0.683 ; p=\mathrm{ns}$. For each cue there 
were eight subjects, only one child missed the pointing trial, two missed a gaze trial, and three missed a glance trial. The girls performed significantly above chance, $t(11)$ $=2.966 ; p=0.013$, the boys did not perform above chance, $t(11)=1.173 ; p=0.266$. However, there was no significant difference between the sexes, $1(22)=0.920 ; p=$ ns. On the second trial only 9 children (as opposed to 18 on the first trial) selected the correct cup, $t(23)=1.238 ; p=$ ns. The children performed significantly better on the first trial than on the second trial, $t(23)=2.584 ; p=0.017$.

However, when $\mathbb{I}$ analyzed the children's choices on the first trial and compared them with the location of the toy on the previous trial (the last orientation trial) I found that 18 of the 24 children selected the cup under which they have seen the toy on the last trial. Only six children selected a different cup then on the previous trial. Remember that the location of the toy on the last orientation trial and on the first experimental trial was counterbalanced per side and cue and sex of the subject. This means that the probability of the toy being in the same or a different location was equal for each of the cues. Al] six subjects that selected a different cup made a correct choice, whereas six subjects that selected the same cup made an incorrect choice.

Next, I analyzed the interaction between the experimenter and the subject from the merged picture of the video recordings. Remember that, with the variable 'looking' I scored whether the subject was looking at one of the cups immediately after the experimenter started presenting the cue. I found that subjects followed the experimenter's cue on $60 \%$ of all trials $(172 / 288)$ by looking at either one of the cups immediately after the cue's onset. On $67 \%$ of the trials $(115 / 172)$, on which the children followed the experimenter's cue, the children responded by looking at the same cup the experimenter was looking at. On the trials on which the children looked at the same cup as the experimenter they chose the correct cup on $73 \%$ of the trials $(84 / 115)$. On $27 \%$ of the trials (31/115) they looked at the correct cup but then chose the incorrect cup. When the children were looking at a different cup or at no cup they chose the correct cup on only $42 \%$ of the trials $(73 / 173)$. When the subjects looked in the other direction than the experimenter they stayed with their (incorrect choice) on $61 \%(35 / 57)$ of the trials, on $39 \%(22 / 57)$ they changed their choice to the correct cup.

Additionally, I analyzed whether the subjects responded to the experimenter's cue by pointing to or reaching for the correct cup, thus making any directional response towards the correct location after onset of the cue. I found that on $19 \%$ of the total number of trials $(56 / 288)$ the children responded to the cue by pointing or reaching towards either of the cups. On the majority of trials $(232 / 288)$ the children did not respond to the cues with pointing or reaching. On $80 \%$ of the trials $(45 / 56)$ on which the children were reaching or pointing towards any of the cups they subsequently chose the correct cup. Additionally, on the majority of the trials on which the children were pointing they chose the cup they were pointing to $(50 / 56)$. On most trials this was the correct cup $(42 / 50)$. On the few trials when children chose another cup than they pointed to $(6 / 56)$ they were equally often
correct as incorrect. 
Further, since the analysis of the subjects" choices on the first experimental trial revealed a perseveration tendency to search for the toy in the location where it has been seen on the previous trial, I compared the subjects' choices of the location of the toy on the previous trial with their correct/incorrect choices. I found that on $60 \%$ of the trials $(164 / 288)$ the children chose the location in which the toy was hidden on the previous trial. An analysis of the actual distribution of locations in the randomized design, remember that the toy was never hidden under the same cup for more than two trials in a row, revealed that the cup was hidden under a different cup than on the previous trial on about $2 / 3$ of the experimental trials (195 versus 93 ). Thus, children chose the same cup slightly more often than a different cup despite the fact that the experimenter was looking at a different cup than on the previous trial on the majority of trials.

\section{DISCUSSION}

In Experiment 2 I was interested in whether young children in contrast to the apes in Experiment 1 would spontaneously use social cues provided by an experimenter to locate hidden objects. Like for the apes in Experiment 1 this task proved to be difficult for the children. Only 5 of the 24 subjects solved the task whereby the pointing cue was the most salient cue for the subjects. Previous studies suggest that children between 2 and 3 years have little problems with using referential cues to find hidden objects (e.g. Itakura \& Tanaka, 1998; Povinelli, Bierschwale, \& Cech, 1999; Tomasello, Call, \& Gluickman, 1997). However, the presentation of the cues in these studies and the meta-procedures used might have made the task easier for the children in these studies.

\section{GENERAL DISCUSSION}

In this study I investigated whether great apes and young children would spontaneously use social cues, such as gaze, glance, and pointing provided by an experimenter to find hidden objects. In particular, I was interested whether our subjects would use the cues without prior exposure to easier cues. The results I obtained with the great apes in Experiment 1 show clearly that our subjects did not understand the referential communicative intent of any of the three cues provided. Although most previous studies come to quite similar conclusions about chimpanzees' ability to use social cues to find hidden rewards (e.g. Call, Agnetta, \& Tomasello, 2000; Call, Hare, \& Tomasello, 1998; Itakura, Agnetta, Hare, \& Tomasello, 1999), there are a few studies that suggest that apes are able to at least understand gaze as a cue to find hidden rewards (e.g. Itakura, \& Tanaka, 1998; Povinelli, Bierschwale, and Cech, 1999; Barth, Reaux, and Povinelli, 2005). So how do we have to explain these results? First of all, it should be noted that the experimental method I had (deliberately) chosen to present the task to the subjects may be the most difficult and demanding design possible. The cues I have chosen each represent the most difficult variation of them. For example, all cues were given in a static manner, for example after the cue was established the experimenter did not look back and forth between the subjects and the cup. Dynamic presentation of 
the cue might increase the salience of the cue. Pointing was administered at chest midline thereby eliminating proximity cues that previous studies have found to be a salient cue for chimpanzees (Povinelli et al., 1997). Moreover, the cues were not preceded by easier cues like touching or tapping the cup which could have helped the apes to develop a general understanding of the task from which they could have generalized to the more difficult cues such as gazing. For example, Povinelli, Bierschwale, \& Cech (1999) and Barth, Reaux, \& Povinelli (2005) first trained their subjects to reliably use a proximate pointing cue before they were advanced to the experimental testing. Additionally, in these studies the proximate pointing cue was used on standard trials in which the experimental trials were embedded. This experimental design of proceeding from a mastered cue to a more difficult cue might have made the task easier for the subjects. Also, the use of easy trials among the experimental trials may have kept the subjects' motivation to pay attention to the task by ensuring that the subjects get rewarded on the majority of trials. Moreover, when a subject enters the test-unit and sees that the experimenter is now doing something different, e.g. giving a gaze cue instead of a proximate pointing cue, may draw the subject's attention to the relevant features of the task. Further, in the present study, I used a randomized design and all trials were experimental trials. So when a subject makes an incorrect choice as a response to a gaze cue it is likely to be confronted with a different cue on the next trial (e.g. pointing). This may minimize the learning effect, because it is more difficult for the subjects to focus on the relevant features of the task. Another crucial difference is that in the present study I did not use the design of a step-wise method. In several previous studies (e.g. Byrnit, 2004; Itakura \& Tanaka, 1998) the subjects were first given an easy cue (like touching the cup) and only after they had mastered this cue were advanced to the next level of difficulty. By using this method, subjects can gradually approach the more difficult cues and subjects that do not pass a difficulty level will not contribute to the data of the next level. How problematic this method can be is illustrated by the results of one of our subjects (Ulla). In the last session Ulla responded correctly to all cues and as the only non-human subject in the study was significantly above chance in a session. However, in the subsequent sessions which were administered on the following days to test how reliable this ability is, her results dropped to chance again. This shows that the results of a single session cannot be taken as a cut-off point for the understanding of a cue and are no convincing evidence that the presumed ability really exists.

All these factors contribute to the high level of difficulty in the experimental design of the present study. However, I chose this design, because I was not interested in the question of whether great apes can eventually learn to use social cues after extensive training, but rather if they would spontaneously use the social cues to find hidden rewards. In conclusion, it seems that the spontaneous use, that is without any prior training, of social cues is beyond the capability of great apes, although it is in general not impossible for apes to successfully use some cues (like proximate pointing, e.g. Povinelli et al. 1997; or even additionally gaze, e.g. Povinelli, Bierschwale, \& Cech, 1999), under curtain experimental conditions. 
Barth, Reaux, \& Povinelli (2005) have recently shown that differences in the experimental design can have a profound effect on the subjects' use of social cues. A recent study by Hare \& Tomasello (2004) also suggests that the ecological validity of the task may play a role. The authors found that chimpanzees used some of the provided cues when they were competing with the experimenter, but not when the experimenter was cooperative like in the present study.

In Experiment 2, I was interested if children, unlike the apes in Experiment 1 , would spontaneously use the cues provided by the experimenter to locate hidden toys. Although, some children of $21 / 2$ year of age successfully used some of the cues (especially pointing) the majority of the children did not find the hidden toys more often than would be expected by chance. This result was somewhat surprising to us as previous studies suggest that children of this age have no difficulties to master this task. For example, Tomasello, Call, \& Gluckman (1997) found no age differences between $21 / 2$ - year old children and 3 - year old children in the use of a pointing cue in an object choice task. At the group level the children found a hidden sticker above chance level by using a pointing cue. However, in this study a proximate pointing cue was used, that is the experimenter was pointing by holding the index finger directly above the cup. In a study by Povinelli et al. (1997) the experimenter used a proximate and a distant pointing cue. In this study, 2 and 3 year-old children were very proficient at exploiting both pointing cues referentially to find hidden rewards, unlike the chimpanzees in this study that relied on proximity cues. Further, Povinelli, Bierschwale, \& Cech (1999) found that 3 - year old children are also able to exploit gazing and glancing cues in a referential manner. The chimpanzees in this study were able to exploit a gaze cue, but, unlike the children, they did so also in situations in which the experimenter was looking above the target and not at the target. Itakura \& Tanaka (1998) tested infants between 18 and 27 months on a variety of cues. The infants used all cues, even glancing, above chance levels, so that the authors concluded from their results that "this task is not difficult for infants older than 18 months" (p. 123). However, also in this study the cues were given in a step-wise design and the pointing cue was given from a distance of only $10 \mathrm{~cm}$ from the correct cup.

Moreover, the present findings are surprising if one considers that children of only 12 months of age, so less than half the age of the children in the present study, actively share interesting sights in their environment with adults (Liszkowski, Carpenter, Henning, Striano, \& Tomasello, 2004). Additionally, several studies suggest that 1-year-olds understand that an adult's gaze towards an object predicts a subsequent action towards that object. Or least children show surprise if adults will act on the object they have previously not been gazing at (Phillips, Wellman, \& Spelke, 2002; Woodward, 2003). The understanding of an adult's intention to act on an object also extends to other cues such as reaching and grasping (Sodian \& Thoermer, 2004).

Our findings in Experiment 2 are possibly compromised by the same factors as Experiment 1 . The children were confronted with a version of this paradigm that was employed in a demanding experimentall setting. Unlike for the 
results of Experiment 1 we have however more variables that will help us to interpret these results. First of all, I analyzed the perseveration errors the children made on their first experimental trial. Recall, that the majority of the children chose the same cup as on the previous trial (the last orientation trial) even though the location of the toy was counterbalanced per cue on the first trial. So the actual probability of the toy being either under the left or the right cup was equal on the first trial. Unfortunately, the location of the food on the first trial in Experiment 1 was not counterbalanced per cue; therefore an analysis of the apes' choices on the first trial was not possible, or at least not meaningful. The fact that the children perseverated the location of the toy from the previous trial indicates that a simple search strategy, searching were the object has been seen before, strongly influenced the children's choice. Some children showed surprise when they saw the experimenter looking at the other cup than where the toy had been seen on the previous trial. After following the experimenter's cue to the correct location, one child commented: "Oh, it's not there!" and pointed to the other cup where the toy had been seen on the previous trial. There is an interesting interplay of social and physical cognition in this $21 / 2$-year old boy's statement. Because it shows how the results of a task that is designed to measure social understanding might be influenced by factors that are unrelated to social cognition. The demands of a manual search task include that a looking response must also be translated into a successful motor response. Hood, Cole-Davies, \& Dias (2003) have shown that $2 \mathrm{l} / 2$ - year-olds, as in the present study, have difficulties searching for an object manually, even if looking measures suggest an understanding of the task. With a simple delayed response task, Hofstadter \& Reznick (1996) haven shown that young infants make more incorrect choices and perseveration errors when searching for objects manually then when their responses are only measured by gaze behavior (see also Zelazo, Reznick, \& Spinazzola, 1998). Perseverative reaching has received much attention in the developmental literature (e.g. Diedrich, Thelen, Smith, \& Corbetta, 2000), but the confounding influences have been largely ignored in object-choice studies, especially in the comparative literature.

In conclusion, the results of this study and the factors which influence them as I discussed them above urge for a further clarification of the object-choice paradigm methodology. Further research should be directed at investigating the influence of a step-wise design versus a randomized design. Moreover, a clear differentiation between cues that can only be solved by understanding the referential meaning and cues that can be solved by other features such as proximity should be made in all studies. Additionally, factors unrelated to social cognition, such as perseverative reaching, should also be considered in future studies using the object-choice paradigm. 


\section{ACKNOWLEDGEMENTS}

I thank Michael Tomasello and Josep Call for helpful discussions and the opportunity to conduct these studies. Il thank Jana Jurkat, Angela Loose, Eva Leermann, Katharina Haberl, and several student assistants for their help in the conduct of this study. I am grateful to the children, parents, and Kindergarten staff for their cooperation. I also thank the keepers and staff at the Wolfigang Kohlier Primate Research Center for their help. 


\section{REFERENCES}

Agneth B., Hare B., Tomasello M. (2000) Cues to food location that domestic dogs (Canis fomiharis) of different ages do and do not use. Animal Cognition, $3,107-112$.

Anderson J. R., Sallaberry $\mathbb{1}^{3}$., Barbier H. (1995). Use of experimenter-given cues during object-choice tasks by capuchin nonkeys. Amimal Behawour, $49,201-208$.

Anderson J. R., Montant M., Schmitt D. (1996). Rhesus monkeys fail to use gaze direction as an experinenter-given cue in ah object-choice task. Behavioral Processes, 37, 47

Barth, J., Reaux, R. E., Povineli, D. J. (2005). Chimpanzes" (Pon troglodytes) use of gaze cues in object-choice tasks: Different methods yield different results. Animal Cogninion, 8, 84-92.

Butterworth, $G_{n}$ \& Jarrett, N. (1991). What minds have in common is space: spatial mechanisms Serving joint wisial attention in infancy. British Journat of Developmental Psychology, 9, 5572.

Byrnit, J. T. (2004). Nonenculturated orangutans" (Pongo pygimaers) use of experimenter- given manual and facial cues in an object-choice task. Nownal of Comparame Psychology, 118 , $309-3.15$.

Call, J. (2004). The use of social information in chimpanzees and dogs. In L. J. Rogers, \& G. Kaplan (Eds.), Comparative Vertebrate Cogmition: Are primates superior to non-primates? (pp. 263286). New York: Kluwer Academic / Plenum Publishers.

Call, J., Agnetta, B., \& Tomaselio, M. (2000). Cues that chimpanzees do and do not use to find hidden objects. Aniwal Cognition 3, 23-34.

Call J., Hare, B., \& Tomasello, M. (1998). Chimpanzee gaze following in an object-choice task. Animal Cognition 1, 89-99.

Call, J., L Tomasello, M. (1994). Production and comprehension of referential pointing by orangutans (Pongo pygmaeus). Journal of Comparative Psychology, 108, 307-317.

Chance, M. R. A. (1967). Attention structure on the basis of primate rank orders. Man, 2, 503-518.

Corkum, W., \& Moore, C. (1995). Development of joint visual attention in infants. In C. Moore \& P. J. Dunham (Eds.), Join Attention (pp. 61-84). Hillsdale, NJ: Lawrence Earlbaum Associates.

Diedrich, F. I., Thelen, E., Smith, L. B., Corbetta, D. (2000). Motor memory is a factor in infant perseverative errors. Developmental Science, 3, 479-494.

Farroni, T., Csibra, G., Simion, F., \& Johnson, M. (2002). Eye contact detection in humans from birth. Proceedings of the National Academy of Sciences, 99, 9602-9605.

Farroni, T., Massaccesi, S., Pividori, D., \& Johnson, M. (2004). Gaze following in newborns. Infancy, 5 , $39-60$.

Fehr, B. J., Exline, R. V. (1987). Social visual interaction: A conceptual and literature review. In A.W. Siegnan and S. Feldstein (Eds.), Nonverbal behavior and commumication (2nd ed.) (pp. 225325). Hillsdale, NJ: Erlbaum.

Hare, B., Brown, M., Williamson, C., Tomasello, M. (2002). The domestication of social cognition in dogs. Science, 298, $1634-1636$.

Hare, B., fomasello, M. (1999) Domestic dogs (Conis familiaris) use human and conspecific social cues to locate hidden food. Journal of Compararive Psychology, 113, 173-177. Hare, B., Tomasello, M. (2004). Chimpanzees ane more skilful in competitive than in cooperative
cognitive tasks. Animal Behowour, 68,571-581.

Hofstadter, M., \& Reznick, J.S. (1996). Response modality affects human infent delayed-response performance. Child Development, $67,646-658$

Hood, B. M., Cole-Davies, V., Dias, M. (2003). Looking and search measures of object knowledge in preschool children. Developmental Psychology, 39, 61-70. Hood, B. M., Willen, J. D.s \& Driver, J. (1998). Adult's eyes trigger shifts of visual attention in human
infants. Psychological Science, $9_{n} 131-134$. Itakura, S. (1996). An exploratory study of gaze-monitoring in nonhuman primates. Japanese
Psychological Research, 38, 174-180.

Itakura, S., Anderson, 1. R. (1996). Learning to use experimenter-given cues during an object-choice task by a capuchin monkey . Cahiers de Psychologie Cogmitive, 15, 103-112.

lakura, S. Agnetta, B., Hare, B., Tomasello, M. (1999). Chimpanzee use of human and conspecific social cues to locate hidden food. Developmental Science, 2,448-456. 
Itakura, S., Tanaka, M. (1998). Use of experimenter-given cues furing object-choice tasks by chimpanzees (Pan troglodyles), and orangutan (Pongo pygmoeus), and human infants (Honos Sapiens). Journal of Comparative Psychology, 112,119-126.

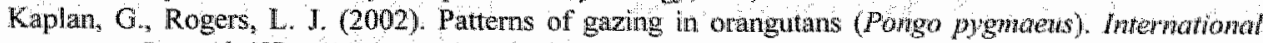
Jownal of Primatolog, 23,501-526.

Lizskowski, U., Carpenter, M., Heming, A., Striano, T., \& Tomaselio, M. (2004). 12-month-olds point to share attention and interest. Developmental Science, $7,297-307$.

Lorincz, E. N., Baker, C. I. Perrett, D. I. (1999). Visual cues for attention following in rhesus monkeys. Cahiers de Psychologie Cognitive, 18, 973-1003.

McKinley, J., \& Sambrook, T. D. (2000). Use of human-given cues by domestic dogs (Comis formiliaris) and horses (Equus caballus). Animal Cogntition, 3, 13-22.

Miklosi, A., Polgardi, R., Topal, J., \& Csanyi, V. (1998). Use of experimenter-given cues in dogs. Amimal Cognition, $1,113-121$.

Myowa-Yamakoshi, M., Tomonaga, M., Tanaka, M., \& Matsuzawa, M. (2003). Preference for luman direct gaze in infant chimpanzees (Pan troglodytes). Cognition, 89, 113-124.

Okamoto, S., Tomonaga, M., Ishii, K., Kawai, N., Tanaka, M. Matsuzawa, T. (2002). An infant chimpanzee (Pan troglodytes) follows human gaze. Animal Cognition, 5, 107-114.

Okamoto, S. Tanaka, M., \& Tomonaga, M. (2004). Looking Back: The "representational mechanism" of joint attention in an infant chimpanzee (Pan troglodytes). Japanese Psychological Research, 46, 236-245.

Phillips, A.T., Wellman. H. M., \& Spelke, E. S. (2002). Infants' ability to connect gaze and enotional expression to intentional action. Cognition, 85, 53.78.

Povinelli, D. J., Eddy, T. J. (1996a). Chimpanzees: Joint wisual attention. Psychological Science, 7, 129-135.

Povinelli D. J., Eddy T. J. (1996b). Factors influencing young chimpanzees' (Pan troghlodytes) recognition of attention. Journal of Comparative Psychology, 110,336-345.

Povinelli DJ, Eddy TI (1997) Specificity of gaze-following in young chimpanzees. British Jowrnal of Developmental Psychology, 15, 213-222.

Povinelli, D. J., Reaux, J. E., Bierschwale, D. T., Allain, A. D., Simon, B. B. (1997) Exploitation of pointing as a referential gesture in young children, but not adolescent chimpanzees. Cognitive Development, 12, 327-365.

Povinelli, D. J., Bierschwale, D. T., Cech, C. G. (1999), Comprehension of seeing as a referential act in young children, but not juvenile chimpanzees. British Journal of Developmental Psychology", $17,37-60$.

Povinelli, D. J., Dumphy-Lelii, S., Reaux, J. E., Mazza, M. P. (2002). Psychological diversity in chimpanzees and humans: New longitudinal assessments of chimpanzes' understanding of attention. Brain Behavior and Evolution, 59, 33-53.

Scaife, M., \& Brunner, J. S. (1975). The capacity for joint visual attention in the infant. Nature, 253, $265-266$.

Scheumann, M. \& Call, J. (2004). The use of experimenter-given cues by South African fur seals (Arctocephalus pusillasi). Animal Cognition, 7, 224-230.

Shapiro, A. D., Janik, V. M., \& Stater, P. I. B. (2003). A Gray Seal's (Halichoerus grypus) Responses to Experimenter-Given Pointing and Directional Cues. Journal of Comparotive Psychology, $117,355-362$.

Sodian, B., \& Thoermer, C. (2004). Infants" understanding of looking, pointing, and reaching as cues to goal-directed action. Joumal of Cognition and Development, 5, 289-316.

Soproni, K., Miklosi, A. Topal, J. \& Csanyi, V. (2001). Comprehension of Human Communicative Signs in Pet Dogs (Canis familiaris). Journal of Comparative Psychology, 115, 122-126.

Tomasello, M., Call, J., Gluckman, A. (1997). Comprehension of novel communicative signs by apes and human children. Child Development, 68, 1067-1080.

Tomasello, M., Call, J., Hare, B. (1998). Fiwe primate species follow the visual gaze of conspecifics. Aninal Behowiour, 55, 1063-1069.

Tomasello, M., Hare, B., Agnetta, B. (1999). Chimpanzees (Pan trogtodytes) follow gaze direction geometrically, Animal Behowiour, 58, 769-777. 


\section{Chapter 2}

Tomasello, M., Hare, B., Fogleman, T. (2001). The ontogeny of gaze following in chimpanzees (Pan troglodytes) and thesus macaques (Macaca wulata). Animal Behowiota, 61, 335-343.

Vick, S-J., Anderson, J. R. (2000). Learning and limits of use of eye gaze by capuchin monkeys (Cebus apella) in an object-choice task. Journal of Comparative Psychology, 114, 200-207.

Woodward, A. L. (2003). Infants' developing understanding of the link between looker and object. Developmental Sclence, 6, 297-311.

Zelazo, P.D, Reznick, J.S, \& Spinazzolla, J. (1998). Representational flexibility and reponse control in a multistep multilocation search task. Developmental PSychology, 34, 203-214. 


\section{CHAPTER 3}

\section{Chimpanzees' use of gaze cues in object- choice tasks: Different methods yield different results}

Published as:

Barth, J., Reaux, J. E., \& Povinelli, D. J. (2005). Chimpanzees' (Pan troglodytes) use of gaze cues in object-choice tasks: Different methods yield different results. Animal Cognition, 8, 84-92. 


\begin{abstract}
In order to assess the influence of differing procedures on chimpanzees" performance in object-choice tasks, five adult chimpanzees were tested using three experimenter-given cues to food location: gazing, glancing, and pointing. These cues were delivered to the subjects in an identical fashion, but were deployed within the context of two distinct meta-procedures that have been previously employed with this species with conflicting results. In one procedure, the subjects entered the test unit and approached the experimenter (who had already established the cue) on each trial. In the other procedure, the subjects stayed in the test unit throughout a session, witnessed the hiding procedure, and waited for a delay of 10 seconds during which the cue was provided. The subjects scored at high levels far exceeding chance in response to the gaze cue only when they approached the experimenter for each trial. They performed at chance levels when they stayed inside the test-unit throughout the session. They scored at chance levels on all other cues irrespective of the procedure. These findings imply that (a) chimpanzees can immediately exploit social gaze cues, and that (b) previous conflicting findings were likely due to the different meta-procedures that were used.
\end{abstract}




\section{INTRODUCTION}

Social cues, such as the orientation of the head and eyes of conspecifics, play an important role in the daily interactions of humans and other social primates (Chance, 1967; Fehr and Exline, 1987). The ability to extract information from conspecifics provides valuable information related to the activities of other members of one"s social group, as well as outsiders (e.g., the presence and position of predators; Povinelli and Eddy, 1996a). Gaze-following, looking where somebody else is looking (Butterwoth and Cochran, 1980; Butterworth and Jarrett, 1991; Scaife and Bruner, 1975), is one mechanism for extracting such information from the behavior of others.

Several species of monkeys and great apes have been shown to follow the gaze of conspecifics and humans (Itakura, 1996; Kaplan and Rogers, 2002; Lorincz, Baker, and Perret, 1999; Povinelli and Eddy, 1996a, 1996b, 1997; Povinelli et al., 2002; Tomasello, Call, and Hare, 1998; Tomasello, Hare, and Agnetta, 1999, Tomasello, Hare, and Fogleman, 2001). Adult and juvenile chimpanzees reliably follow a human's gaze when it is signalled by eye movements alone (Povinelli and Eddy, 1996b), even when eye movements and head movements are dissociated (Povinelli et al., 2002). Although the exact ontogeny of gaze-following in chimpanzees and other primates is unclear, this topic is currently under investigation (e.g, Ferrari, Kohler, Fogassi, and Gallese, 2000; Okamoto et al., 2002; Okamoto, Tanaka, and Tomonaga, 2004).

Despite the widespread agreement that chimpanzees (and other species) spontaneously follow the gaze of others, there is considerably less agreement about the ability to use gaze direction to extract certain kinds of social information. Does following a human or conspecific's gaze direction indicate an understanding of communicative intent of the signal, or are such responses caused by mechanisms unrelated to such social understanding? One experimental technique for exploring this question has come to be known as the object-choice paradigm. In this paradigm, an experimenter typically hides food in one of two locations and then provides a social cue (e.g. gazing, glancing, or pointing) to the food location.

The object-choice paradigm has been used with a number of non-human primate species. Capuchin monkeys, for example, have shown the ability to use experimenter-given cues to locate food, but they have only done so in response to a proximate pointing cue that included the experimenter's hand resting on the traty and the index finger being only 15 centimeters away from the cup (experiments 3 and 5, Anderson, Sallaberry, and Barbier, 1995). Similar findings have also been reported for rhesus monkeys (Anderson, Montant, Schmitt, 1996). Itakura and Anderson (1996) trained a capuchin monkey to use several cues as tapping, pointing, and gazing. Although the subject acquired the skill to use the cues it required considerable training to do so and the cues again were presented quite proximal to the reward location. Furthermore, the monkey failed to learn to use glancing as a cue. Moreover, the distant gazing cue was presumably only acquired because it was presented in a step-wise fashion by presenting the distant cue $(60 \mathrm{~cm})$ after the 
proximate cue $(15 \mathrm{~cm})$ had been mastered by the subject. In an additional study, Vick and Anderson (2000) demonstrated that for capuchin monkeys head orientation is a more salient cue than eye direction. These studies highlight that monkeys do not readily use experimenter cues but can learn to do so after extensive training.

Research with great apes has produced a less clear pattern of results. Itakura and Tanaka (1998) reported that two chimpanzees and one orangutan acquired the skill to use five different cues (including glancing) when they were presented in sessions of increasing difficulty. Tomasello, Call and Gluckman (1997), on the other hand, reported that chimpanzees did not reliably use cues (even a proximate pointing cue) to locate a rewarded location. Similarly, Itakura, Agnetta, Hare and Tomasello (1999) reported that chimpanzees were unsuccessful at using a combined point/gaze cue to locate the rewarded location (although they performed better when a gaze cue was supplemented with a vocalization). Call, Hare, and Tomasello (1998) showed that although chimpanzees were following the experimenter's gaze into space above and behind them, they did not use this information to locate food hidden under one of two bowls (although changing the type of the occluder slightly enhance the subjects' performance). In another study, Call, Agnetta, and Tomasello (2000) found that various kinds of noises facilitated chimpanzees' ability to use a gazing but not a glancing cue. Nonetheless, when the authors combined a gazing cue with several behavioral cues (like touching or lifting the cups) most of the chimpanzees could not find the food at above chance levels. Finally, in an object-choice study with three species of great apes, Barth, Call, \& Tomasello (unpublished data) found that none of the apes reliably used any of the social cues that were presented (gaze, glance, and point).

In contrast to these findings, Povinelli, Bierschwale, and Cech (1999) reported immediate success by their chimpanzees in exploiting a gaze cue. The authors were interested if their subjects would spontaneously generalize their proficiency at using a trained pointing cue to other social cues (such as gazing and glancing). They first trained their chimpanzees to reliably respond to a proximate pointing cue to determine if they could transfer this knowledge to gaze and glance cues. On these transfer tests, the chimpanzees exhibited above-chance performance in the gaze condition that was stable from trial one forward. They did not successfully exploit the glancing cue. Furthermore, unlike young 3-year-old children who were tested using the same method, the chimpanzees also used the gazing cue even when the experimenter did not directly gaze at the target but above and behind it. This finding suggests that despite their trial one success, in contrast to the human children, the chimpanzees were relying on a gaze-following response that directed them in the general direction of the correct cup, not an understanding of the communicative intent of the gaze.

The discrepant findings from chimpanzees tested in different laboratories on the ability to exploit a distal gaze cue warrants further attention, especially with regard to the role of subject versus procedural factors. The history, ages and gender 
of the subjects of these studies have been variable, as have been the metaprocedures used. To address this problem, we used a within-subjects design to compare the procedure used in the study by Povinelli, Bierschwale and Cech (1999) with procedures used in other studies (e.g. Cal], Hare, and Tomasello, 1998; Barth, Call, \& Tomasello, unpublished data). By using the successful subjects of Povinelli, Bierschwale and Cech (1999), we were able to test the hypothesis that the discrepant results were the result of procedural, not subject factors. Therefore we modeled our procedures as closely as possible after studies that have previously been conducted. In this study it was not our intention to tease apart which details of a procedure may influence the subjects' behavior.

\section{METHOD}

\section{Subjects}

Seven adult chimpanzees, housed in a social group at the University of Louisiana at Lafayette, Louisiana, participated in this study. They ranged in age from $13 ; 2$ to $14 ; 1$ years when the experiment began. Five of the subjects had been peer-raised in a nursery setting, whereas two of the subjects (Apollo and Megan) had been raised by their mothers during the first year of life (before joining the nursery group). The subjects were housed in one group, but tested individually in a setting and using procedures that have been described in detail elsewhere (see Povinelli, 2000). The animals had participated in numerous other studies involving joint attention, the interpretation of social cues (such as the direction of eyes, head, and body, and pointing), as well as numerous studies of the understanding of physical causation (see Povinelli, 2000).

\section{Procedure}

Overall design. The chimpanzees were tested for their ability to use three social cues to locate a food reward: gaze, glance, and midline pointing. Each subject was tested using the exact same cues as presented in the context of two distinct meta-procedures. These two procedures will be referred to as the (1) LEAVE procedure (modeled after Povinelli, Bierschwale and Cech, 1999) and the (2) STAY procedure (modeled after Barth, Call, \& Tomasello, unpublished data), to reflect one of the most salient differences between the methods (see Table 1 for a detailed comparison of the two procedures).

Testing environment. The subjects were tested individually in an environment that consisted of an outdoor waiting area connected by a shuttle door to an indoor testing unit. When the subjects entered the testing unit they were separated from experimenters by a large, Plexiglas wall, through which they could respond. They could enter and leave for each trial (LEAVE) or remain in the test unit as each new trial was set up (STAY). 
TABLE 1. Methodological differences between STAY and LEAVE procedures.

\begin{tabular}{|c|c|c|}
\hline \multirow[b]{2}{*}{ Procedural feature } & \multicolumn{2}{|c|}{ Procedure } \\
\hline & STAY & LEAVE \\
\hline Sulject postition & $\mathrm{S}$ stays in test-unit for all trialls & $S$ enters and leaves for each trial \\
\hline Training criterion & $\begin{array}{l}\text { S trained to select cup that is } \\
\text { wisually baited }\end{array}$ & $\begin{array}{l}S \text { trained to select cup to which } E \\
\text { points }\end{array}$ \\
\hline Food hiding & $\begin{array}{l}\text { S observes } E \text { hiding food, but not } \\
\text { exact location }\end{array}$ & $S$ does not hiding process \\
\hline Transfer cup & $\begin{array}{l}\text { Third cup used to transfer food in } \\
\text { hiding process }\end{array}$ & $N / A$ \\
\hline Test trial presentation & Test trials presented in blocks & $\begin{array}{l}\text { Test trials as probes blocks amid } \\
\text { pointing trials }\end{array}$ \\
\hline Cue presentation & $\begin{array}{l}\text { S awaits each cue onset after hiding } \\
\text { process }\end{array}$ & Cue available to $S$ at trial onset \\
\hline Cue duration & $\begin{array}{l}\text { Cue stops before } \mathrm{S} \text { is allowed to } \\
\text { respond }\end{array}$ & Cue present as $\mathrm{S}$ responds \\
\hline Subject control & $\begin{array}{l}\text { S must wait to respond until } 10 \\
\text { seconds after cue onset }\end{array}$ & S can respond to cue at any time \\
\hline
\end{tabular}

Apparatus, Each meta-procedure required a different experimental set-up. For LEAVE, a table $(147 \mathrm{~cm} \times 30 \mathrm{~cm} \times 37 \mathrm{~cm})$ was used to present the task to the subjects. The table was placed $40 \mathrm{~cm}$ in front of the Plexiglas partition (Figure 1a). Two identical, square cups ( $10 \mathrm{~cm}$ diameter, $14 \mathrm{~cm}$ high), were used to hide the food. The cups were hinged to the table top and were separated by $91 \mathrm{~cm}$. The apparatus was positioned so that the subjects could enter and directly reach through either a right or left response hole in the Plexiglas barrier and push over one of the cups. After the subjects had responded, the response holes could be remotely closed
to prevent a second choice. 

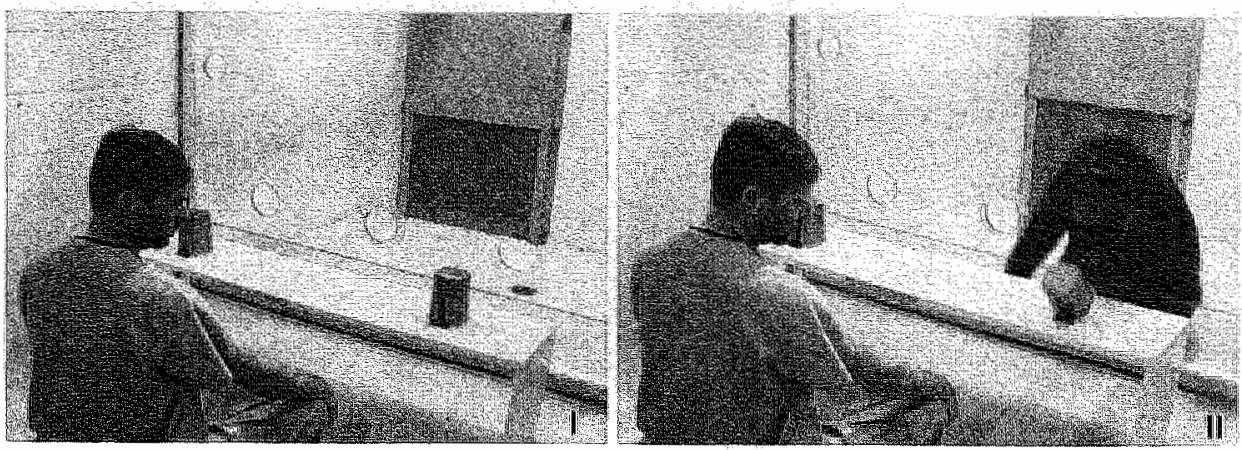

FIGURE 1A. Photographs of the test-unit showing the LEAVE procedure. In LEAVE, the subject enters and leaves the test-unit for each trial. (I) The experimenter establishes the cue before the subject enters. (II) The subject approaches the experimenter after entering the test-unit and chooses one of the two cups.

For STAY, a table $(122 \mathrm{~cm} \times 61 \mathrm{~cm} \times 40 \mathrm{~cm})$ was used to present the task to the subjects. The table was placed against the Plexiglas partition (Figure 1b). A panel $(108 \mathrm{~cm} \times 41 \mathrm{~cm})$ resting on top of the table was used to slide the task back and forth. Three opaque cups were used. A small cup $(5 \mathrm{~cm}$ in diameter and $6 \mathrm{~cm}$ in height) was used to contain the reward (grapes, slices of banana, or juice). Two identical cups ( $10 \mathrm{~cm}$ diameter, $12.5 \mathrm{~cm}$ high) were used to hide the food cup. Two small barriers were fastened to the outer comers of the tray to ensure that the two cups were always placed in the exact same location $(91 \mathrm{~cm}$ apart). On test trials, a rectangular cover $(38 \mathrm{~cm} \times 38 \mathrm{~cm} \times 15 \mathrm{~cm}$ ) was used to prevent the subjects from seeing under which cup the food was hidden. A. Plexiglas panel with two small holes $(4 \mathrm{~cm}$ diameter) covered the larger holes in the Plexiglas barrier thereby allowing the subjects to respond only by inserting a finger into the hole corresponding to the cup in front of it.

Orientation. The subjects received orientation sessions to familiarize them with the different set-ups and general methods for each procedure. The subjects were randomly divided into two groups $(n=3, n=4)$. In the first session, one group received orientation for STAY and the other for LEAVE. In the second session, the procedures were reversed between groups, and alternated thereafter. For both types of procedures, orientation consisted of 6 trials per session, during which the reward was hidden under each cup on half of the trials. The order of locations was randomized within the constraint that the food was never under the same cup more than twice in a row. Each subject received at least 2 sessions (one per procedure) each consisting of 6 trials. A minimum of 5 correct choices out of 6 trials per procedure was set as a criterion to advance to Testing. 

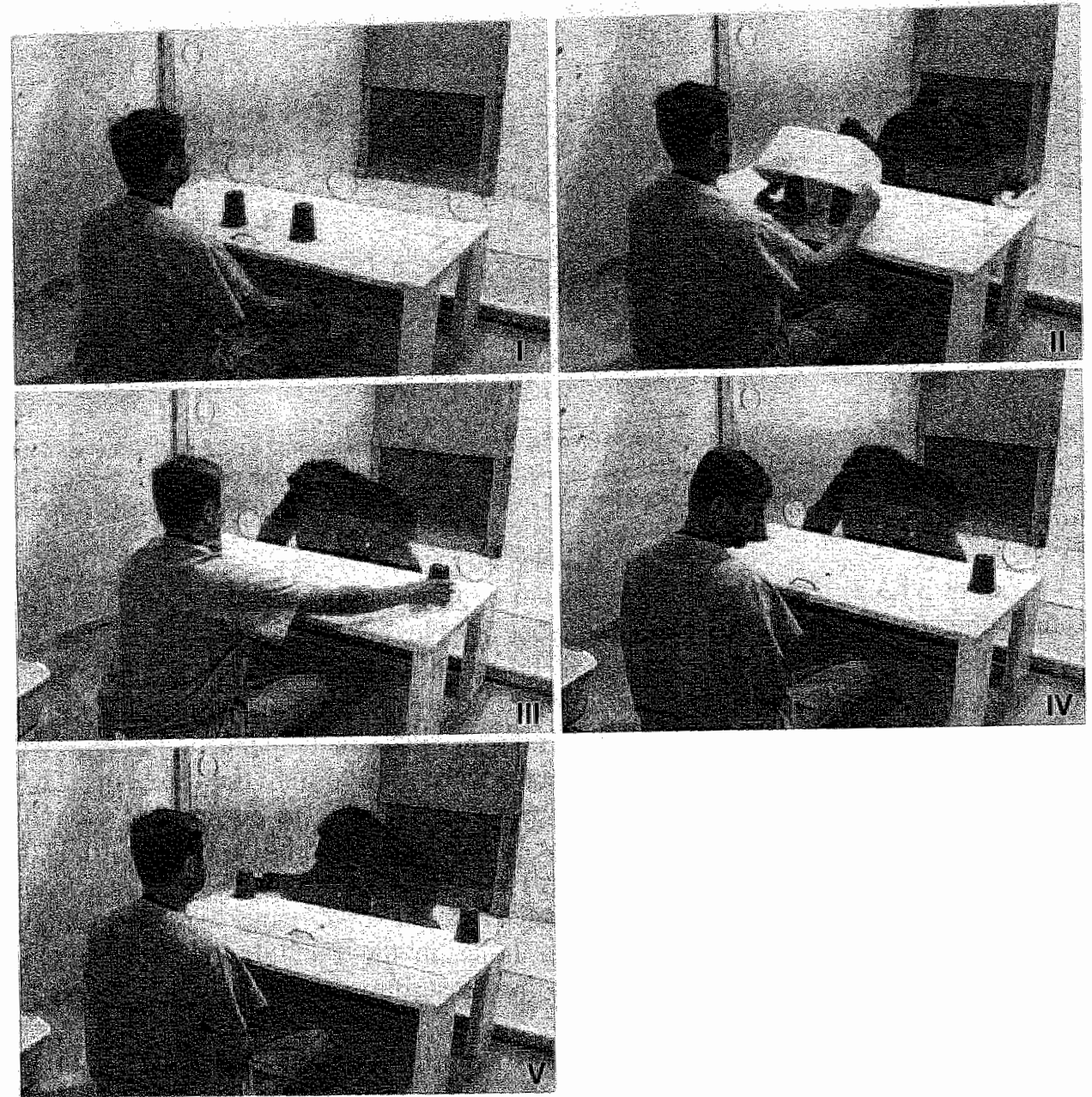

FIGURE IB. Photographs of the test-unit showing the STAY procedure. In STAY, the subject stays inside the test-unit throughout a session. (1) The experimenter places the cups on the pulled back tray white the subject is inside the test-unit. (II) He then conceals the cups and hides food under one of them. (Iii) The cups are placed into their positions and (IV) the cue is established and given for 10 seconds. (V) The experimenter then looks straight ahead and pushes the tray forward allowing the subject to choose one of the cups.

Orientation continued until the subjects met criterion on two consecutive sessions. The specific purpose of LEAVE orientation sessions was to ensure that the subjects would correctly respond to a previously learned proximal pointing cue (see Povinelli, Bierschwale and Cech, 1999). At the start of each trial, the subject waited outside while the experimenter placed a piece of food under one of the two cups. The experimenter then sat on the floor behind the table (so as to be at eye level with 
the subject) and pointed to the baited cup with extended arm (with the tip of the index finger approximately $5 \mathrm{~cm}$ away from the cup). The arm used corresponded to the side of the cup. An assistant opened the shuttle door and closed it behind the subject once he or she entered, allowing the subject to enter and approach the Plexiglas barrier and respond. The subject was allowed to make only one choice (defined as pushing over one of the cups). After each trial, the assistant re-opened the shuttle door, let the subject back into the outdoor waiting area, and closed the shuttle door so the next trial could be prepared. This process of entering and leaving the test unit was repeated for each trial.

The specific purpose of STAY orientation sessions was to be certain the subjects would correctly gesture to a cup that they witnessed being baited. Each trial began by opening the shuttle door and allowing the subjects to enter the testunit (where they remained for the duration of the session). The two large response cups were placed on the sliding tray (out of the subject's reach) that rested on the table. Once the subject was attentive to the task the experimenter baited the small food cup, showed it to the subject and placed it in the middle of the tray. The experimenter then showed the subjects that the hiding cups were empty and positioned them upside-down with the food cup being in the middle. He then placed one cup over the food cup and slid the two cups into their final positions on the tray. The experimenter used a beeper with an ear-plug to count a delay of 10 seconds. During the dellay, the experimenter looked straight ahead with a neutral face. After the 10-second delay, the experimenter slid the tray up to the Plexiglas barrier so that the subject could respond by poking at one of the cups. A choice before the end of the delay was ignored, unless the finger was still resting in the Plexiglas hole when the tray was slid forward. Once the subject made a choice, the selected cup was lifted and the tray was pulled back. Once the tray was pulled back the second cup was lifted. If the subject had chosen the cup containing the food the subject was handed the food item. If not, the next trial began.

Testing. All subjects met criterion for LEAVE, but only 5 of the 7 subjects met criterion for STAY. Because of the within-subjects design, only these 5 subjects were advanced to testing. They were randomly assigned to two new groups $(\mathrm{n}=2, \mathrm{n}=3)$. The subjects received blocks of the LEAVE and STAY testing sessions in a counterbalanced fashion. Thus, one group began testing with a block of LEAVE sessions, the other group started with a block of STAY sessions. For LEAVE, each block consisted of 6 sessions of 8 trials each (of which 2 were probe trials and 6 were standard proximal pointing trials). The probe trials were always administered on trials 3 and 6 . For STAY, each block consisted of 2 sessions of 6 trials, each of which were probe trials. Within a session all 3 cues were given twice (once to the left and once to the right).

For both procedures, during probe trials, the experimenter administered one of three cues to the cup containing the food: (1) gaze (head + eyes), (2) glance (eyes only), and (3) point (looking straight ahead, pointing finger at shoulder level in middle of chest). The cues were delivered in the exact same manner in both LEAVE 
and STAY by two experimenters whose participation was equated by condition and cue. The type of the cue and the location of the food were randomized and counterbalanced so that the same Jocation was never correct on more than two consecutive trials. For STAY, an additional constraint was imposed so that within a session, for each cue type, the food was in the left and right locations an equal number of trials.

During LEAVE testing, two types of trials were administered: (1) standard trials, in which the experimenter pointed to the baited cup with extended arm and his finger $5 \mathrm{~cm}$ away from the cup just as during the orientation sessions, and (2) probe trials, in which the experimenter established one of the three cues (gaze, glance, or point) directed to the baited cup before the subject entered. All cues were given until the subject had chosen. Otherwise, the general set-up and procedures during testing were the same as for the orientation sessions.

During STAY testing, the experimenter used a large opaque cover to prevent the subject from witnessing the hiding procedure. During the hiding procedure, the experimenter made the same sound and movement with both cups to prevent the subject from extracting any auditory or motion cues. When the food was hidden and the cups were slid into their position, the experimenter gave one of the three cues to the cup containing the food, as soon as the subject was attentive. Each cue was given for a duration of 10 seconds. After the delay, the experimenter looked straight ahead with a neutral face and pushed the tray forward allowing the subject to choose. The subject was allowed to make only one choice. The experimenter then proceeded as in the orientation sessions and administered the next trial.

\section{Videotape coding and reliability assessment}

Orientation sessions were independently coded on-line by two assistants. Testing sessions were coded from video records by two observers not involved in acquisition of the data or any other part of the experimental phase of this study. The main observer rated all testing sessions of all five subjects. For "cup-choice" and "hand-use' a second observer rated all testing sessions of two randomly selected subjects (Apollo and Candy). For 'premature cup-choice during the delay in STAY', a second rater coded all STAY trials of all animals. The inter-observer agreement was calculated using Cohen's Kappa $(\kappa)$. Only the coding results of the main observer where used in the data analysis. Three variables from the testing sessions were coded: (1) the cup the subject selected either by turning it over (LEAVE) or by touching it through the hole in the Plexiglas (STAY), for which raters agreed on $99 \%(239 / 240)$ of the trials $(\kappa=0.99)$, (2) the hand the subject used to make its choice, for which raters agreed on $99 \%(238 / 240)$ of the trials $(\kappa=0.98)$, and (3) the "premature choices" of subjects during the cue presentation period in STAY (response holes through which the subject protruded a finger before the cue presentation period was completed), for which raters agreed on $88 \%(104 / 120)$ of
the trials $(\kappa=0.79)$. 


\section{Orientation}

\section{RESULTS}

For LEAVE, the subjects that participated in the study required an average of 7 sessions (range 4-13) to reach criterion. For STAY the subjects that participated in the study $(n=5)$ needed an average of 16 sessions (range 9-29) to meet the required criterion of a minimum of 5 correct choices out of six trials to advance to Testing. Subjects that did not show any improvement received a stepwise introduction to STAY in which they were verbally encouraged to respond to the task. However, to reach criterion these subjects had to pass the initial version of the STAY orientation (without any verbal prompting). Two subjects (Jadine and Mindy) did not show any improvement across 21 and 26 sessions, respectively, and were therefore not advanced to the test phase.

\section{Testing}

Cup-choice. Table 2 shows the mean percentage of correct trials per cue for each procedure. A Friedman Test revealed an overall effect of condition $\left(\chi_{5}^{2}=14.1\right.$, $\mathrm{p}=0.015$ ). A comparison of the results by cue by procedure depicted in Figure 2 shows that the gaze cue in LEAVE was the only cue the subjects reliably used to find the hidden food. All 5 subjects scored 100 percent correct in this cue/condition, interpreting the gaze cue in LEAVE correctly on all 8 trials. A Wilcoxon paired ranks test showed that all 5 subjects performed significantly better in the gaze condition in LEAVE than STAY, or any other condition, irrespective of the procedure (all $\mathrm{p}<0.05$, two-tailed). (The p-values have been adjusted for multiple testing by using Hochberg's improved Bonferroni method; Hochberg, 1988, Schaffer, 1995.) Next, we compared the subjects' performance to chance. The only experimental cue in which the subjects (as a group) selected the correct cup at above chance levels was the gaze cue in LEAVE (binomial test, $P=.50, p<0.001$, two-tailed). The subjects chose the correct cup during the standard pointing trials in LEAVE on $351 / 360$ trials ( $97.5 \%$ correct).

Premature cup-choice during the delay in STAY. The subjects indicated a choice during the delay (by protruding their finger through one of the response holes before the cups were slid to within reach) on $54 \%$ of the trials in STAY. Of these trials, the subjects selected the same cup during the delay as after the delay on $69 \%$ of the trials. On $54 \%$ of the trials the premature choice was the correct choice. Thus, the subjects were also at chance levels in their 'premature' choices. 
TABLE 2. Mean Percentage of Correct Trials by Subject, Cue, and Procedure.

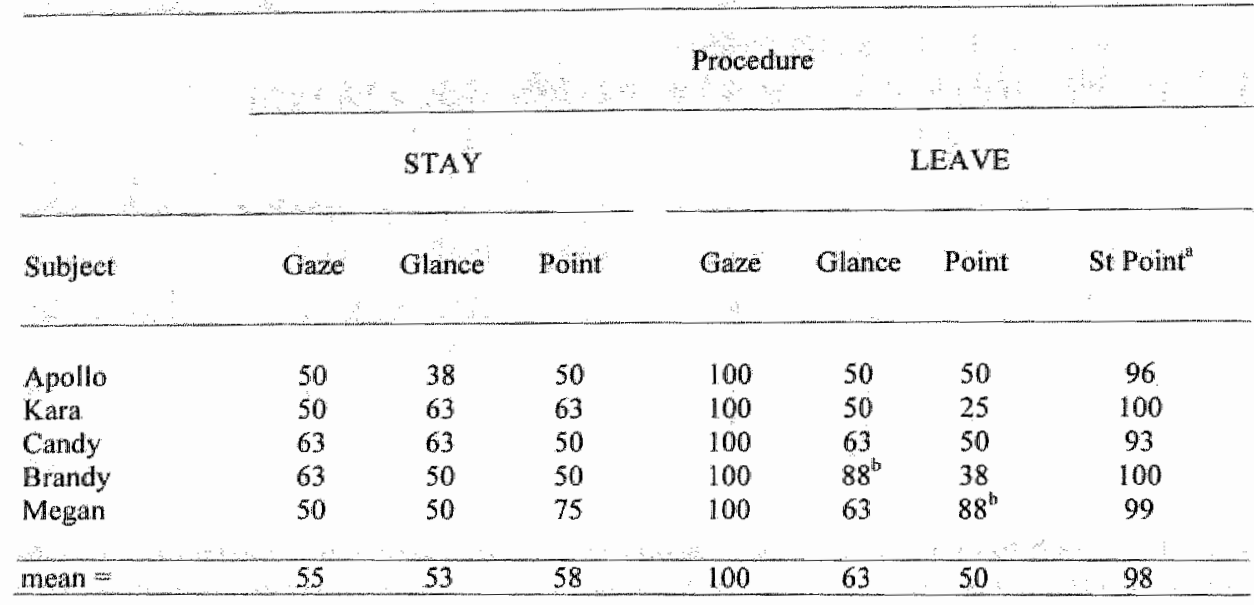

Notes. "Proximal pointing trials used on standard trials in the LEAVE procedure; "p $p<.05$, Binomial tiest.

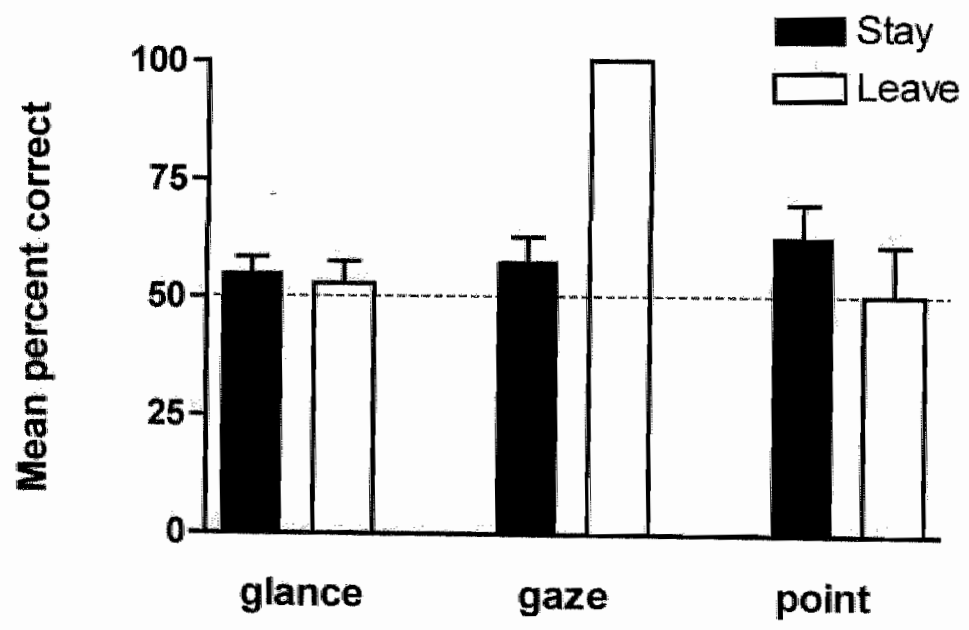
FIGURE 2. Mean percentage of correct trials $(+/-\mathrm{SD})$ per cue and procedure. Each graph represents
the mean score of 40 trials ( 8 trials per subject). 
Hand-use. Table 3 shows the Directional Handedness Index (HI) (after Hopkins, 1999) for each subject per procedure. Different preferences in hand-use between the procedures may indicate, at a simple behavioral level, that the responses of the subjects were influenced by the procedures. One subject showed a strong preference to use his right hand to select the cups irrespective of the procedure (Apollo). Another subject showed the same pattern for the left hand (Candy). Kara did not show any preference for either hand irrespective of the procedure. However, for both Brandy and Megan the preferred hand to make their choice was dependent on the procedure, indicating that for these two subjects, at least, the different procedures significantly affected a rather fundamental aspect of their behavior.

Procedure

\begin{tabular}{|c|c|c|c|c|c|c|}
\hline \multirow[b]{3}{*}{ Subject } & \multicolumn{2}{|c|}{ STAY } & \multicolumn{4}{|c|}{ LEAVE } \\
\hline & \multicolumn{2}{|c|}{ Probe Trials } & \multicolumn{2}{|c|}{ Probe Trials } & \multicolumn{2}{|c|}{ Standard Trials } \\
\hline & $\mathrm{HI}$ & Pref & $\mathrm{HI}$ & Pref & $\mathrm{HI}$ & Pref \\
\hline Apollo & -0.84 & $\mathrm{R}$ & -1 & $\mathbb{R}$ & -1 & $\mathrm{R}$ \\
\hline Kara & -0.16 & A & -0.16 & A & 0 & $\mathrm{~A}$ \\
\hline Candy & 0.92 & L & 1 & $\mathrm{~L}_{\mathrm{i}}$ & 1 & L \\
\hline Brandy & -1 & $\mathrm{R}$ & -0.08 & A & 0.06 & A \\
\hline Megan & 0.08 & A & 0.92 & L & 1 & L. \\
\hline
\end{tabular}

TABLE 3. Directional Handedness Index (HI) per subject, cue, and procedure. Nole Directional Handedness Index (HI) per subject, cue, and procedure. HI was calculated by dividing the difference between the percentage of left and right hand-use by their sum $(L-R) /(L+\mathbb{R})$. Neggative vallues indicate a tendency to use the right hand, whereas positive walues indicate a tendency to use the lef hand (e.g., HII $=1$ represents exclusive use of the left hand on all trials). $L=$ left-handed; $\mathbb{R}=$ righthanded; $A=$ ambidextrous (after Hopkins, 1999).

Archival analysis of learning effects. Finally, in order to determine if our subjects' ability to exploit the gaze cue in LEAVE was learned, we compared the archival data from the three previous studies in which LEAVE had been used with these five subjects. Two of these studies are reported in Povinelli, Bierschwale and Cech (1999), and one is from an unpublished study (conducted approximately 3 
years after the first two studies) archived in the Cognitive Evolution Group data archives (available upon request).'

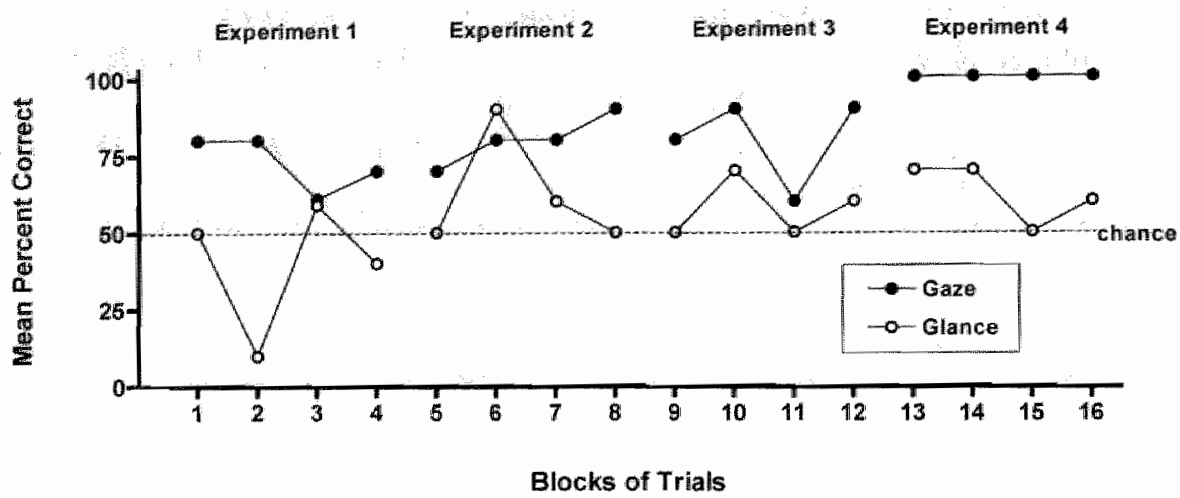

FIGURE 3. Learning curves for the gaze and gllance cues across four consecutive experiments. The data are from 3 previously conducted experiments (blocks $1-12$; see text) and the present experiment (blocks 13-16). Each data point represents the grand mean score of 5 subjects (2 trials per subject).

These data, along with those from the present study, are plotted in Figure 3. Although the subjects do exhibit some improvement across experiments in response to the gaze cue, as can be seen, the subjects were above-chance from the very first block of two trials, and maintained a high performance level across each subsequent replication. In contrast, comparable improvement is not seen in response to the glance cue. (Although the fundamental stability of performance in response to the gaze cue indicates it was not a factor, it should be noted that there was one difference across these experiments in how the cues were presented. In Exp. 1, as in the present experiment, the cues were presented statically, with the experimenter gazing or glancing at the correct location. In Experiments 2 and 3, the cues were presented dynamically, with the experimenter moving his or her head or eyes back and forth between the subjects and the correct location.)

\section{DISCUSSION}

This study investigated the influence of two different experimental procedures on chimpanzees" use of three social cues in an object-choice task. All five subjects selected the correct cup when the experimenter gazed at it, but they were only able

\footnotetext{
'It should be noted that other studies involving these subjects" use of the gaze oues to locate objects, using different procedures, were conducted between the first two experiments of Povinelli et al. (1999) and the archived replication study (see Povinelli et al. 1997, and Povinelli et al. 2002). These published data merely reinforce our point concerning the subjects' initial and stable above-chance performance in responding to the gaze cue using the LEAVE method.
} 
to do so in one of the procedures (LEAVE). This difference is all the more striking given that all five subjects selected the correct option on all $100 \%$ of the gaze trials in the LEAVE procedure. The other cues (pointing, glancing) were not reliably used in either procedure. Given the counter-intuitive nature of these results (i.e., that such a seemingly small procedural difference could have such a large impact on the subjects" performance), as well as the implication that our results have for previous research that has not used the LEAVE method, in what follows we critically examine several key issues concerning both the present study, as well as previous studies in this area.

First, it could be argued that the ease with which the subjects used the gaze cue in LEAVE was due to their previous experience with this procedure (e.g, Povinelli, Bierschwale, and Cech, 1999). However, it is unlikely that the history of our subjects explains the differential results we obtained between the STAY and LEAVE methods in the present study. As can be seen in the results of Experiment 1 in Figure 3, our subjects were able to exploit the gaze cue from the very first time they were confronted with it (see Povinelli et al., 1999). The effect of the subjects' experience with the gaze cue in the LEAVE method only explains the increase in performance from $80 \%$ correct trials on their first encounter with the LEAVE method in the 1999 study to the $100 \%$ correct trials in the present study, not their initial (and stable) ability to exploit the cue. Further, the subjects did not show any improvement in using the LEAVE glance cue (pointing was not included in the previous study, but see Povinelli et al. 1997). Finally, it is important to reiterate that these same subjects were unable to exploit an identical social cue in the STAY procedure. This strongly suggests that what we have described as the STAY procedure may mask abilities that are in fact present.

Second, with respect to the subjects' chance-level performance in the STAY procedure, we examined whether the subjects had made a correct choice during the delay, but then changed their choice because the experimenter did not: immediately respond. The results clearly show that even though the subjects did, in fact, make a response during the delay on more than half of the trials, their choices were as often correct as incorrect. Therefore, by itself, the delay in the STAY procedure is unlikely to explain the subjects' chance-level performance.

Third, the results confirming the subjects' lack of use of the glance (eye movement alone) and midline pointing cue may be explained by the salience of these cues. In the case of the glancing cue, although chimpanzees show a gazefollowing response based on eye direction alone (Povinelli and Eddy, 1996a), in the context of an object choice task glancing might not be a salient enough cue to direct the subject into the direction of the correct cup. In the case of the midline pointing cue, previous studies have suggested that chimpanzees rely on proximity features of pointing cues (Povinelli et al., 1997). Presumably, for the chimpanzees a pointing cue with an index finger that is equidistant to both cups, so that proximity cues are neutralized, does not draw the chimpanzees' attention preferentially toward one of the two locations. 
Fourth, although further studies are necessary to exactly isolate the methodological factors that allow chimpanzees to exploit the gaze cue in one method but not the other, at present the approach towards the experimenter in the LEAVE method would seem to be the most likely factor. In a previous study (Povinelli et al, 1999) it was found that chimpanzees, unlike children, allso use a gazing cue that is not directed at the cup, but above the cup (above-target condition). This finding was interpreted as reflecting that the chimpanzees were directed in the general direction of the correct cup by their natural gaze-following response when approaching the experimenter, in contrast to an understanding of the communicative content of a cue that creates a triadic connection. The results of the present study can be interpreted in a similar fashion. In the LEAVE procedure, the subjects entered the test-tinit and, while approaching the experimenter and apparatus, their automatic responses to the experimenter's fixed head and eye orientation directed their attention (and hence primed their responses) toward the position of the correct cup. The STAY procedure, in contrast, may limit the subjects' abilities to exploit this cue because of attentional factors related to repeated movements and incidental orientations on the part of the experimenter as each trial is being prepared. In this context, the change of hand preference that we observed in two of our subjects also suggests that the subjects changed to a different response mode during the STAY procedure. A shift in hand preference from standard trials in the probe trials of the STAY but not the LEAVE procedure may reflect uncertainty or confusion.

Finally, to the best of our knowledge, the LEAVE method in the present study (along with the previous studies cited above involving these subjects) differs from all other research on the ability of chimpanzees to exploit human cues in the object-choice paradigm in that the subjects approach the experimenter on each trial. However, despite the fact that we showed that these subjects (a) responded in a robustly different manner to the STAY and LEAVE methods and (b) that this is unlikely to be the effect of differential experience with the LEAVE method, it will be important to replicate our findings with a group of naivve subjects. Also, because no studies have been conducted with any species of monkeys using the LEAVE method, it would be of great interest if monkeys, like our apes; were able to exploit an experimenter's gaze cue using this method without requiring extensive training. Thus, given the increasing use of object-choice tasks that involve experimentergiven social cues, our results suggest that greater attention should be devoted to the exact methods used within this paradigm. Our results open the possibility that some methods used in previous studies might have masked an ability to exploit humangiven cues (regardless of the underlying cognitive processes supporting that ability).

This attention should be extended to non-primate species, as well. For example, with respect to the recent results suggesting that dogs are superior to apes in using experimenter-given cues (e.g. Hare, Brown, Williamson, and Tomasello, 2002; Hare and Tomasello, 1999; Soproni, Miklosi, Topal, and Csanyi, 2001), it would be crucial to evaluate the effect of different methods on the dogs' performance. In these studies, the subjects typically approach the experimenter 
from a distance (i.e., the LEAVE method). It would be of interest to know if they perform equally well if they are sitting in front of the experimenter. After all, our results show that in response to certain cues, apes can be highly adept at exploiting them, so long as the correct meta-procedure is used. The effect of these different methods should also be ruled out when comparing dogs with wolves (e.g. Hare, Brown, Williamson, and Tomasello, 2002), especially because wolves were tested from behind a fence meshing and, unlike the dogs, were not approaching the experimenter. That is, the dogs were tested using the LEAVE method, whereas wolves have been tested using the STAY method. As we have shown here, it is problematic to draw conclusions about performances across these two metamethods.

Finally, a comparison between these methods should also be conducted with human subjects. The few studies that have been conducted with children all showed that children around 3 years of age are successful in using social cues to find hidden objects (e.g. Itakura, \& Tanaka, 1998; Povinelli et al., 1997; Povinelli, Bierschwale, \& Cech, 1999; Tomasello, Call, \& Gluckman, 1997). The procedures used in these studies were similar or comparable to either the LEAVE or the STAY procedure. This would suggest that for children the ability to use social cues does not depend on the meta-procedure.

In conclusion, future studies testing the ability of animals to exploit humangiven social cues in object-choice tasks should display greater sensitivity to the meta-methods that are used, and take these methods into consideration when interpreting the results across different species, as well as across different groups of the same species tested in different laboratories.

\section{ACKNOWLEDGEMENTS}

We thank Anthory Rideaux, Luke Brean, and Amy Roberthon for crtelal help in conducting this study, We also thank seweral undergraduate students for their assistance of various kinds in the conduct of this study. Further, we are grateful to four anonymous reviewers for their helpful comments on an artios versilon of this manuscript. The research reported in this article was approved by the Uriversity of Louisiana at Lafayette Institutional Animal Care and Use Committea and was conducted in acoordance with all applicable laws of the Unites States of America. The work was funded by dames $\mathrm{S}$. McDonnell Centennial Fellowship Award to DIP. 


\section{REFERENCES}

Agnetra $\mathrm{B}_{\text {. Hare }} \mathrm{B}$, Tomasello M (2000) Cues to food location that domestic dogs (Conis familiaris) of different ages do and do not use. Arim Cogn 3, $107-112$.

Anderson $J \mathbb{R}$, Sallaberry P. Batbier $H$ (1995) Use of expermenter-given cues during object-choice tasks by capuchin monkeys. Anim Behav 49, 201-208.

Anderson JR, Montant M, Schmit D (1996) Rhesus monkeys fail to use gaze direction as an experimenter-given cue in an object-choice task. Behav Proc 37,47-55.

Butterworth $G^{3}$ Cochran $E(1980)$ Towards a mechanism of joint visual attertion in human infancy. Int I Behav Dev $3: 253-272$.

Butterworth $G$, Jartet $N(1991)$ What minds have in common is space: spatial mechanisms serving joint visual attention in infancy. Br I Dev Psychol, 9:55-72.

Call J. Agnetta B, Tomasello M (2000) Cues that chimpanzees do and do not use to find hidden objects. Anim Cogn 3, 23-34.

Call J, Hare B, Tomasello M (1998) Chimpanzee gaze following in an object-choice task. Anim Cogn 1 , $89-99$.

Chance MRA (1967) Attention structure on the basis of primate rank orders. Man 2, 503-518.

Fehr BJ, Exline RV (1987) Social visual interaction: A conceptual and titerature review. In AW Siegman and S Feldstein (Eds.), Nonverbal behavior and communication (2nd ed.) (pp. 225325). Hillsdale, NJ: Erlbatum.

Ferrari $\mathrm{PF}$ Kohler $\mathrm{E}$, Fogassi $\mathrm{L}$, Gallese $\mathrm{W}(2000)$ The ability to follow eye gaze and its emergence during development in macaque monkeys. Proo Nat Acad Sci 97, 13997-14002.

Hare B, Tomasello M (1999) Domestic dogs (Canis familiaris) use human and conspecific social cues to locate hidden food. J Comp Psychol 113, 173-177

Hare $B_{i}$ Brown M, Williamson C, Tomasello M (2002) The domestication of social cognition in dogs. Sci $298,1634-1636$.

Hocliberg $Y$ (1988) A sharper Bonferroni procedure for multiple tests of significance, Biometrika 75 , $800-802$.

Hopkins WD (1999) On the other hand: Statistical issues in the assessment and interpretation of hand preference data in nonhuman prinates. International J Primatol 20, 851-866.

Itakura S (1996) An exploratory study of gaze-monitoring in nonluuman primates. Japan Psychol Res 38 , $174-180$.

Itakufa S, Anderson IR (1996) Learning to use expertmenter-given cues during an object-choice task by a capuchin monkey. Cahiers de Psychologie Cognitive, 15, 103-112.

Itakura S, Agnetta B, Hare B, Tomasello M (1999) Chimpanzee use of human and conspecific social cuess to locate hidden food. Dev Sci2,448-456

Itakura $S$, Tanaka, M (1998) Use of experimenter-given cues during object-choice tasks by chimpanzeos (Pan troglodones), and orangutan (Pongo pygmaeus), and human infants (Homo Sapienas). J Comp Psychol 112, 119-126.

Kaplan G, Rogers LJ (2002) Patterns of gazing in orangutans (Pongo pygmaeus). Intern J Primatol 23 , $501-526$.

Lorincz EN, Baker CI, Perrett DI (1999) Visual cues for attention following in thesus monkeys. Cahiers de Psychologie Cognitive 18,973-1003.

Okamoto $S$, Tomonaga $M$, Ishii $K$, Kawai $N$, Tanaka M, Matsuzawa T (2002) An infant chimpanzee (Pan roglodyfes) follows human gaze. Anim Cogn 5, 107-114.

Okatnoto $S$, Tanaka $M$ \& \& Tononaga M (2004) Looking Back: The "representational mechanism" of joint attention in an infant chimpanzee (Pan troglochtes). Jap Psychol Res 46, 236-245.

Powinelli DJ, Eddy "TJ (1996) Chimpanzees: Joint visual attention. Psychol Sci 7, 129-135.

Povinelli D., Eddy TI (1996b) Factors influencing young chimpanzees' (Pan troglodytes) recognition of attention. ol Comp Psychol $110,336-345$.

Povinelli DJ, Eddy TI (1997) Specificity of gaze-following in young chimpanzees. Br J Dev Psychol 15, $213-222$

Povinelli DJ, Reaux JE, Bierschwale DT, Allain AD, Simon BB (1997) Exploitation of pointing as a referential gesture in young children, but not adolescent chimpanzees. Cogn Dev 12, 327-365. 
Powinelli DJ, Bierschwale DT, Cech CG (1999) Comprehension of seeing as a referental act in young children, but not juvenille chimpanzees. Br I Dew Psychol 17, 37-60.

Povinelli DJ, Dumphy-Lelii S, Reaux JE, Mazza MP (2002) Psychological diversity in chimpanzees and humans: New longitudinal assessments of chimpanzees' understanding of attention. Brain Behav and Evol 59, 33-53.

Scaife M, Bruner JS (1975) The capacity for joint visual attention in the Hinfant. Nature 253:265-266.

Schaffer JP (1995) Multiple hypothesis testing. Annual Rev Psychol 46.561 -584.

Tomasello M, Call J, Gluckman A (1997) Comprehension of novel communicative signs by apes and human children. Child Dev 68, 1067-1080.

Tomasello M, Call J, Hare B (1998) Five primate species follow the visual gaze of conspecifics. Anim Behav 55, $1063-1069$.

Tomasello M. Hare B, Agnetta B (1999) Chimpanzees (Pan troglodytes) follow gaze direction geometrically. Anim Behav 58, 769-777.

Tomasello M, Hare B, Fogleman T (2001) The ontogeny of gaze following in chimpanzees (Pan troglodytes) and rhesus macaques (Macaca mulatta). Anim Behav 61, 335-343.

Vick S-J, Anderson IR (2000) Learning and limits of use of eye gaze by capuchin monkeys (Cebus apella) in an object-choice task. J of Comp Psychol 114, 200-207. 



\section{III \\ EXPLORING ALARMING OBJECTS BY USING TOOLS}




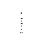

$\vdots$

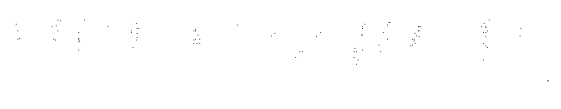




\section{CHAPTER 4}

\section{Tool-mediated object exploration by chimpanzees: Implications for the evolution and acquisition of human tool- use}

Submitted for publication as:

Barth, J., \& Povinelli, D.J. Tool-mediated object exploration by chimpanzees:

Implications for the evolution and acquisition of human tool-use. 


\begin{abstract}
Researchers have long puzzled over species differences in the ability to use tools, and in particular why chimpanzees (and certain other great apes and humans) exhibit such an extensive natural tool-kit unlike any other primates. To approach this topic experimentally, we investigated a particular type of tool-using behavior in chimpanzees related to body-image. Here we show that chimpanzees spontaneously recruit a tool to mediate interactions with alarming objects, but not with unthreatening objects. Drawing on recent findings concerning the neural representations underlying tool-use, we suggest that an explicit representation of the body, unique to humans and great apes, can explain the unparalleled tool-using abilities shown by these species.
\end{abstract}




\section{INTRODUCTION}

In the wild, chimpanzees make and use tools to extend their reach, to probe for food, to wield as simple weapons, to amplify the force of their own actions (e.g., nutcracking), and for sponging up liquids (1, 2). By the Plio-Pleistocene, hominids had developed stone tools, complex weapons, and a controlled use of fire (3-5). Anthropoid monkeys and prosimians, on the other hand, exhibit little tool-use in the wild, although they can be trained on simple tool-using tasks in captivity (e.g. raking in food rewards) (6). Species differences in the neuronal representations underlying tool-use may account for this diversity. In studies of the neuronal representation of the body, researchers have distinguished between body schema, a system for implicit proprioceptive information like body posture and movement, and body image, a system that represents more explicit knowledge about the body, like awareness of body parts, and provides information about the body that is available for cognitive processing $(7,8)$. What happens with these body systems during tool-use? One idea, proposed for the first time almost a century ago, is that tools become incorporated into the implicit body schema and are represented as an extension of the hand (9-11). Recent neuroscientific research has confirmed and extended this idea $(12,13)$. For example, studies in monkeys have shown that the representation of the hand by visual-tactile neurons is extended to include the tip of a tool after learning to use a tool as a rake (14). Similar observations have been reported for humans (15). Moreover, this research shows that after tools are integrated into the body schema, visual space that was previously coded as far (out of the hand's reach) is now coded as near (within the hand's reach with the help of a tool) $(16,17)$.

Although body schema is involved in learning to use tools in human and nonhumans alike, humans additionally possess an explicit body image. Independent evidence suggests that great apes also possess at least some subset of this system (18-20). A body-image system may allow more flexible forms of tool use and facilitate their discovery through the awareness of manual actions on objects and an explicit representation of the distinction between hand and tool. To investigate a tool-using behavior that would be facilitated by body image (as opposed to body schema), we studied the chimpanzee's capacity for tool-mediated object exploration. This can be defined as preferentially using a tool (e.g., a stick) as opposed to the hand to mediate contact with an alarming or potentially dangerous object. This form of tool-use is also shown by humans, for example, when using tongs or pokers when interacting with fire. This behavior requires an explicit representation of the body, since the tool is not used to extend one"s reach but to substitute for bodily contact with a dangerous object. This is qualitatively different from use of tool as a rake, which merely requires a representation of the tool as an extension of the hand. Surprisingly, to the best of our knowledge, there has so far been no systematic study of this special form of tool-use (21).

We studied this behavior experimentally by capitalizing on the ability of six wellstudied adult chimpanzees to use stick tools to retrieve out-of-reach food rewards 
(22). Chimpanzees are an ideal population for studying this form of tool-use, because among non-human primates they show the greatest variety and flexibility in the use of tools (6). Moreover, chimpanzees are widely regarded as the best available comparative model for the evolution of hominid of tool-use $(23,24)$. Finally, chimpanzees (and other great apes) exhibit other behaviors (e.g. selfrecognition) that indicate the presence of a more explicit body image than that found in other primates (25).

\section{METHOD}

Seven adult chimpanzees (age range $11 ; 2-12 ; 1$ years) living in a social group at the Cognitive Evolution Group initially participated in this study. One subject (Jadine) completed all testing, but was excluded from the final analysis because she showed neither hand nor tool use in all three conditions when an AO was present, therefore providing no data for the hypothesis under examination. Details of their living and testing environment and their experience with using tools can be found in ref. 22 . A wooden box $(46 \times 40 \times 28 \mathrm{~cm})$, open on one side, was constructed to present the task to the subjects. Sixteen different objects, (rubber snakes, stuffed animals, etc.), were selected based on criteria known to be aversive to chimpanzees (e.g. furriness, having eyes). Their aversive effect was informally confirmed prior to testing with another group of chimpanzees. Light-blue colored cotton cloths were used to conceal the box. Before the subjects were advanced to testing we first administered several sessions to confirm the subjects' ability to rake in distant food rewards on standard retrieval trials. The subjects were required to use one of the tools to rake in a cookie placed on the floor. Six subjects met the criterion of four consecutive successful trials within their first session. Another subject (Mindy) did not exceed three successful trials per session but was advanced to testing after 25 sessions. For the position of the box (within reach) and the position of the reward (out of reach) on orientation and standard trials the maximum reach was measured for each individual prior to the study to assure a correct positioning for each subject. For each trial, the box was covered with a cloth. After the subject entered the test unit the cloth was either immediately remotely removed by the experimenter (uncovered box condition) or not removed (covered box condition). The subjects were tested across 12 sessions. Each session consisted of four standard retrieval trials (mean attempt rate $=100 \%$, mean success rate $=82 \%$ ) and two test trials. All sessions were recorded on video. Two observers were asked to rate how many bouts the subjects made to the food or the object with either hand or tool. One observer rated all trials of all subjects. The second observer rated all trials of two subjects. Inter-observer reliability was calculated by using Cohen's Kappa. Agreement between the observers was excellent $(k=.90)$, therefore only the coding results of the first observer were used in the analysis.

First, we let our chimpanzees observe an experimenter uncover a small box that was positioned so that its open side faced the subject. On two of these familiarization trials the box contained food (e.g. an apple), and on two it contained 
an alaming object (AO) (e.g. rubber snake). Next, three kinds of test trials were presented to the chimpanzees: $(A)$ food alone $(n=4)$ or $A O$ alone $(n=4)$ with box covered, $(B)$ food alone $(n=4)$ or $\mathrm{AO}$ alone $(\mathrm{n}=4)$ with box uncovered, and $(\mathrm{C})$ mixed (both food and object present) with box uncovered; with two sub-conditions: food and alarming object side-by side $(n-4)$, food behind the alaming object $(n=$ 4). On all test trials the box was positioned so that the contents were easily within the subject's hand reach. On each trial, three identical stick tools (length $=50 \mathrm{~cm}$ ) were present (always in the same location). Figure 1 shows the testing environment for each type of the test trials.
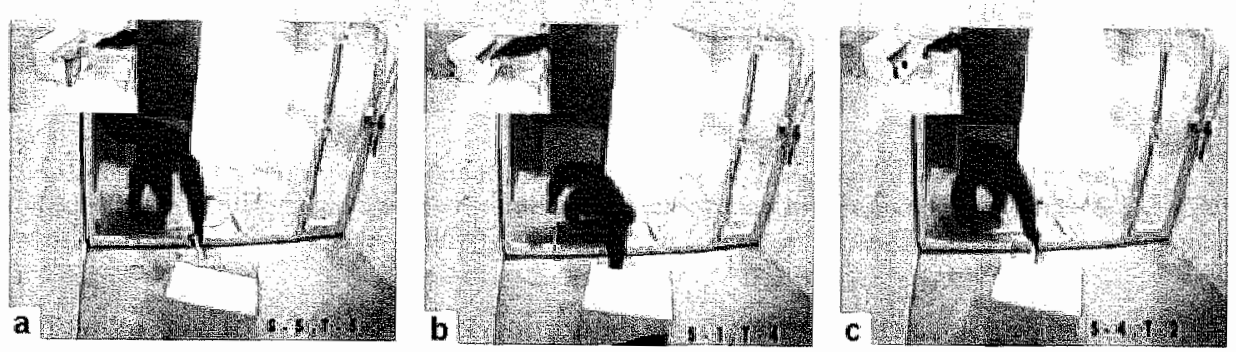

FIGURE 1. Still pictures of the video recordings showing each of the three kinds of test trials. (a) frood or alarming object alone with box covered. The subject uses a tool to uncover the box. (b) Food or alarming object alone with box uncovered. After the cloth has been removed by the experimenter the subject picks up a piece of food from the box. The three provided tools remain untouched. (c) Food and alarming object with box uncovered. After the cloth has been removed by the experimenter the subject uses a tool to move the alarming object away from the food.

\section{RESUILTS}

Condition A was critical, because the subjects could not know what was in the box until after they removed the clloth (using either a hand or tool). Figure 2 reveals that three subjects invariably used the tool to remove the cloth ( $M=7.5 / 8$ trials), whereas the other three showed the opposite pattern of using their hands to remove the cloth $(M=7.5 / 8$ trials $)$. By itself, this shows that some of our chimpanzees spontaneously used the tools in a context in which they could not be certain of the potential threat (26). Importantly, this behavior was related to their behavior in the other two conditions. Subjects who uncovered the box by using a tool also used a tool significantly more often than the other subjects when contacting the $A O$ when only it was present, $(t=6.634, d f=4, p=0.003)$, and significantly more often to contact the $A O$ when both $A O$ and food were present $(t=7.402, d f=4, p=0.002)$. When no AO was present, all subjects exhibited comparable scores for hand use. 


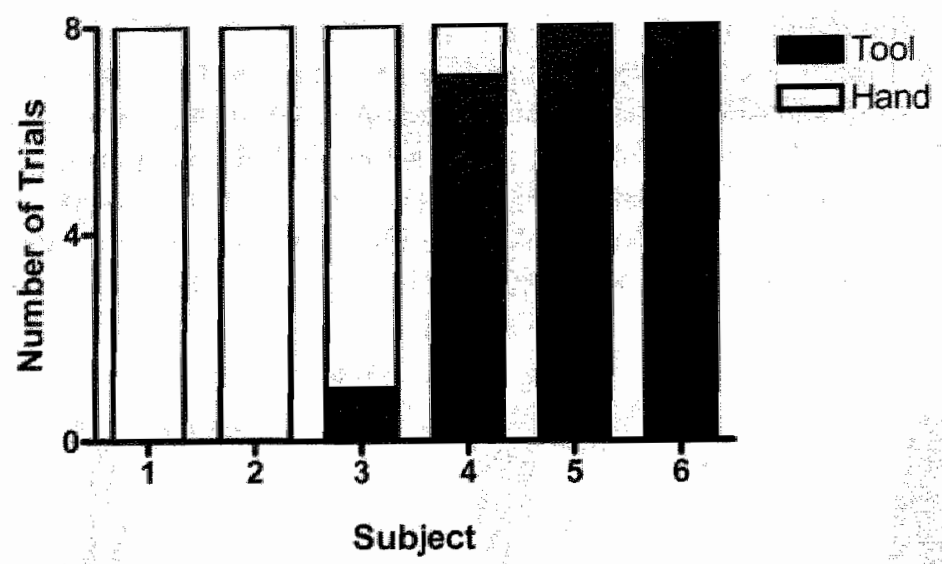

FIGURE 2. Hand wersus tool-use for uncovering the box in the box covered condition. The bars show the number of trials a subject used the tool (black) or the hand (white) to remove the cloth from the box.

Next, we used a Pearson correlation to determine if the use of tool versus hand in condition A predicted behavior in the other conditions when an $A O$ was present. There was a significant positive correlation between the percentage of trials that a subject used the tool to uncover the box, and the percentage of bouts a subject used the tool to contact an $\mathrm{AO}$ when only an $\mathrm{AO}$ was present $(\mathrm{r}=0.95, \mathrm{n}=6, \mathrm{p}=0.003)$ and when both $A O$ and food were present $(r=0.97, n=6, p=0.001)$. That is, the more a subject used the tool in Condition A (when they were uncertain of the box's contents), the more likely he or she was to use a tool (and not the hand) to contact AOs in the other conditions. We label the three subjects that selectively used a tool in conditions involving (or potentially involving) AOs, 'context-dependent toolusers" (CDTUs), and the remaining subjects, who showed the opposite pattern of behavior, 'simple tool users' (STUs).

A comparison between conditions showed that when only food was present, both groups used their hands to retrieve the food rather than a tool on more than $90 \%$ of the bouts, a difference significantly above chance (STUs: $t=6.500, \mathrm{df}=2$, $p=0.023$; CDTUs: $t=7.604$, $d f=2, p=0.017$ ). In condition $B$, however, when only an $\mathrm{AO}$ was present, the groups showed strikingly different patterns: STUs still used the hand more often than the tool $(\mathrm{t}=3.555, \mathrm{df}=2, \mathrm{p}=\mathrm{ns})$ touching the object on almost $75 \%$ of the bouts, whereas CDTUs used the tool significantly more often than the hand $(\mathrm{t}=5.750, \mathrm{df}=2, \mathrm{p}=0.029)$, contacting the object with their hands on less than $8 \%$ of the bouts (Figure $3 a$ ). Similar differences emerged in the (mixed) condition C: STUs used the hand on a mean of $80 \%$ of the bouts to contact either food or object $(t=7.604, \mathrm{df}=2, \mathrm{p}=0.017)$, whereas CDTUs used the tool as much as the hand to make contact with the food, and significantly more often to contact 
the object $(t=11.000, \mathrm{df}=2, \mathrm{p}=0.008)$, overall touching the $\mathrm{AO}$ on less than $5 \%$ of the bouts in condition $\mathrm{C}$ (Figure $3 \mathrm{~b}$ ):

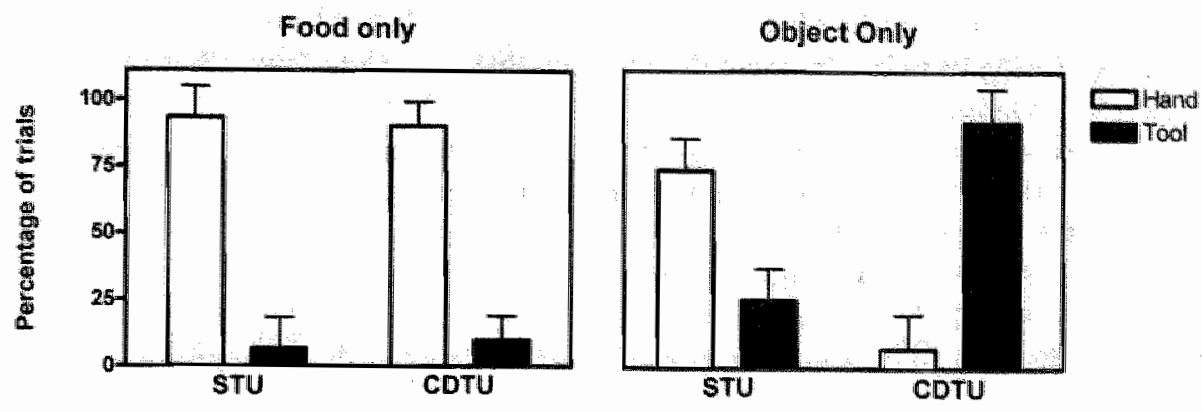

FIGURE 3A. Mean percentage of tool and hand bouts $(+s . d$.) per group of subjects. The left graph shows the mean percentage of tool and hand bouts when only food was inside the box. The right graph shows the mean percentage of tool and hand bouts when only an object was inside the box.
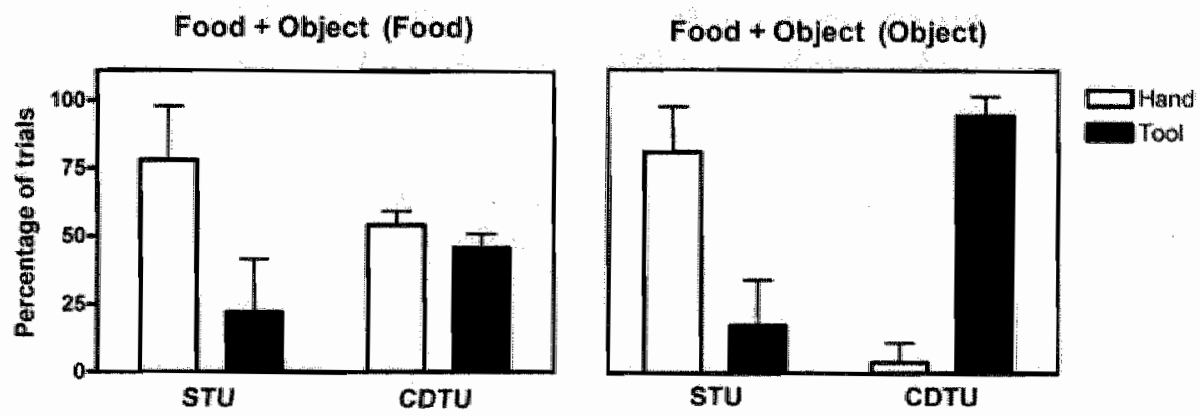

FIGURE 3B. Mean percentage of tool and hand bouts $(+s . d$.) per group of subjects. The left graph shows the mean percentage of tool and hand bouts the subjects made to the food when both food and an object were inside the box. The right graph shows the mean percentage of tool and hand bouts the subjects made to the object when both food and an object were inside the box. STU, simple tool-users; CDTU, context dependent toolusers.

\section{DISCUSSION}

These results show that chimpanzees can selectively use tools rather than their hands to mediate interactions in situations involving (or potentially involving) alarming objects. This suggests that chimpanzees, like humans, possess an explicit body image that facilitates a sophisticated form of tool-use not yet shown by other animals. These findings have important implications for the study of the mechanism, development, and evolution of various types of tool-use. First, they should stimulate neuroscientists to broaden their investigations of the neurobiological basis of tooluse to include the distinctive contributions of body schema and body-image. Second, 
although development of tool-use in human infants and other primates has typically been conducted within the context of the development of causal understanding (22), developments in body-related knowledge may be correlated with the kinds of context-dependent tool-use inwestigated here. It would be of great interest to know how these forms of tool-use develop, and how they correlate with other measures of explicit knowledge about the body (e.g., body self-reeognition) $(25,27)$. Third, one of the most important cultural breakthroughs in human history was the controlled use of fire (28). The representational system we have studied here maybe one of the prerequisites that enabled hominids to use fire for their benefits in a number of important ways that shaped human evolution dramatically. Finally, the distinctions drawn here, coupled with new assays for explicit knowledge about the body, may offer insights into understanding why tool-use seems so dramatically advanced in great apes and humans, as opposed to other primates (29).

\section{ACKNOWLEDGMENTS}

We thanlk James E. Reaux, Anthony Rideaux, Laura Theall, Sarah Dunphy-Lelii, and Luke Brean for cricial assistance during the conduct and analysis of this study. The research reponted in this articte was approved by the University of Lousisiana at Lafayette Institutional Animal Care and Use Committee and was conducted in accordance with all applicable laws of the Unites States of America. The work was funded by a James $\$$. McDonnell Centennial Fellowship Award to DJP. 


\section{REFERENCES}

1. W.C. McGrew, Chimpanzee Material Culture: Implications for Human Evolution (Cambridge Univ. Press, New York, 1992).

2. J. Goodall, Chimpanzees of Gombe: Patterns of Behavior (Harvard Uniw. Press, Cambridge, 1986).

3. K.D. Schick, \& N. Toth, Making Silent Stones Speak. Human Ewolution and the Dawn of

Technology (Touchstone, New York, 1993).

4. H. Knecht, Projectile Technology (Plenum, New York, 1997).

5. S.R. James, Curr. Anthropol. 30, 1 (1989).

6. B.B. Beck, Animal Tool Behavior (Garland, New York, 1980).

7. S. Gallagher, J Mind \& Behav, 7, 541 (1986).

8. J. Cole, J. Paillard, in The Body and the Self, J. Bermudez, A. Marcel, N. Eillan, Eds. (MIT Press, Cambridge, 1995).

9. H. Head et al., Studies in Neurology (Frowde \& Hadder, London, 1920).

10. J. Piaget, The Construction or Reality in the Child (Ballantine, New York, 1954).

11. J. Paillard, in The Use of Tools by Human and Non-human Primates, A. Berthelet, J. Chavaillon, Eds. (Clarendon, Oxford, 1993).

12. S. Johnson-Frey, Trends Cogn. Sci. 8, 71 (2004).

13. A. Maravita, A. Iriki, Trends Cogn. Sci. 8, 79 (2004).

14. A. Iriki, M. Tanaka, Y. Iwamura, Neuroreport 7, 2325 (1996).

15. A. Farnê, E. Làdavas, Neuroreport 11, $1645(2000)$.

16. A. Berti, F. Frassinetti, J. Cogn. Neurosci. 12, 415 (2000).

17. A. Maravita, M. Husain, K. Clarke, J. Driver, Neuropsychologia 39, 580 (2001).

18. G. G. Ir. Gallup, Science 167,86(1970).

19. D.J. Povinelli, J.G.H. Cant, Q. Rev. Biol. 70, 393 (1995).

20. S. Kojima, Primates 44, 231 (2003).

21 . A few aneedotal observations of tool-mediated object exploration by great apes have been reported.

See Ref 6.

22. D.J. Povinelli, Folk Physics for Apes (Oxford Univ. Press, New York, 2000).

23. W.C. McGrew, in Tools, Language and Cognition in Human Exolution. K.R. Gibson, T. Ingold, Eds. (Cambridge Univ. Press, New York, 1993).

24. N. Toth, K.D. Schick, S.E. Savage-Rumbaugh, R. Sevcik, D. Rumbaugh, J. Archaeol. Sci. 20, 81 (1993).

25. D.J. Povinelli, A.B. Rulf, K.R. Landau, D.T. Bierschwale, J. Comp. Psychol. 107, 347 (1993).

26. The individual variation among our subjects may reflect individual variations that have been uncovered in other domans that atre facilitated by a body inage, such as mirror self-recognition (see ref $25,27)$.

27. M.W. de Veer, G.G. Jr. Gallup, L.A. Theall, R. van den Bos, D.J. Provinelli, Neuropsychologia 4I, $229(2003)$.

28. J. Mccrone, New Scientist 166, 30 (2000).

29. W.C. McGrew, in Comparative Socioecology: The Behavioural Ecology of Hunans and Other Mammals, V. Standen, R.A. Foley, Eds. (Biackwell, Oxford 1989). 



\section{CHAPTER 5}

\section{Bodily Origins of SELF}

Published as:

Barth, J., Povinelli, D.J., \& Cant, J.G.H. (2004). Bodily Origins of SELF. In D. Beike, J. L. Lampinen, \& D. A. Behrend (Eds.), The Self and Menory (pp. 11-43). New York: Psychology Press. 


\section{INTRODUCTION}

Thirty-five years ago, Gallup (1970) reported the existence of a surprising phylogenetic difference in the capacity of organisms to recognize themselves in mirrors: the ability appeared to be present in chimpanzees, but not in several species of monkeys that he tested. After being exposed to mirrors, he observed chimpanzees apparently using their reflected images to explore parts of themselves that they had never had the opportunity to see before (their eyes, teeth, nose, and ano-genital area, for example). Figure 1 provides some examples of these behaviors captured in our own laboratory. To confirm the interpretation of these spontaneous reactions, the chimpanzees were anesthetized and surreptitiously marked with a red dye on the upper portion of their eye brow ridge and ear (locations that were only visible with the aid of a mirror). Upon recovery, the chimpanzees made no attempt to touch these areas until they were again allowed to observe themselves in a mirror, at which point they immediately began touching the marked regions of their faces (see also Povinelli et al., 1997). Gallup concluded that the chimpanzees had learned to recognize themselves in mirrors. Gallup (1970) also tested several species of Old World monkeys using the same procedures. Surprisingly, the monkeys, although initially interested in the mirror, neither exhibited the selfexploratory behavior, nor touched the red spots on their faces during the mark test.

Reports from a number of laboratories replicated Gallup's (1970) findings with chimpanzees, and continued to fail to find evidence for mirror self-recognition in lesser apes and a wide array of other non-human primates (review by Anderson \& Gallup, 1999). Other research extended the presence of the capacity for mirror self-recognition to another great ape species, orangutans, but somewhat surprisingly, not gorillas (e.g., Suarez \& Gallup, 1981). More recently, the final species of great ape, bonobos (Pan paniscus) has been tested and reported to exhibit evidence for mirror self-recognition, as well (although these latter tests have lacked important controls; Hyatt \& Hopkins, 1994; Walraven, Elsacker \& Verheyen, 1995).

\section{THE BODY IN THE MIRROR}

As time passed, and evidence continued to suggest a robust difference in the capacity for self-recognition in mirrors between chimpanzees, orangutans and humans, on the one hand, and other primates on the other, Gallup (e.g., 1977) increasingly favored the provocative conclusion that differences in the capacity of organisms to recognize themselves in mirrors might reflect differences in the presence of self-awareness, what he defined as the ability to become the object of one"s own attention. His common-sense interpretation of the results was that it was impossible to know who it was you were seeing in a mirror unless you first had a concept of self. Later, Gallup (1982) further speculated that if chimpanzees and orangutans were self-aware in this sense, they might also have the capacity to reflect upon their own experiences and, by inference, the experiences of others as well. 

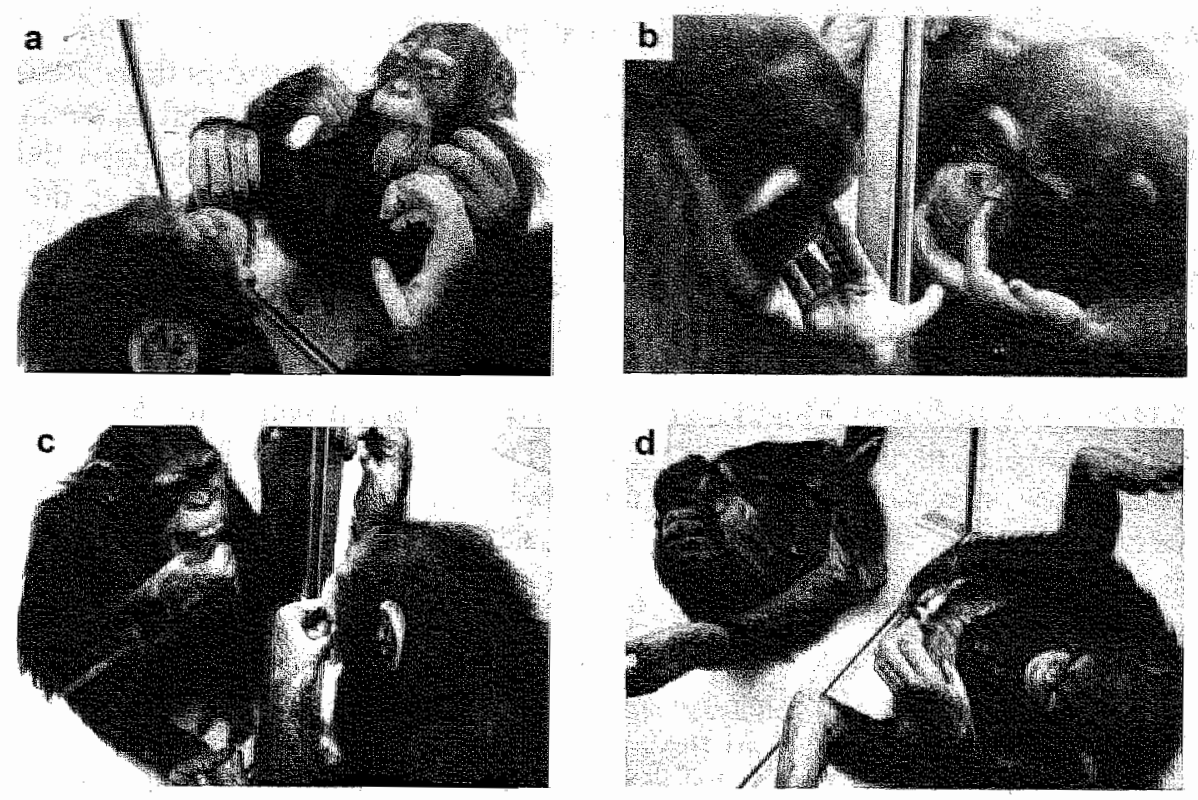

FIGURE 1 A 51/3-year-old chimpanzee (a) exhibiting exaggerated facial expressions and (b-d) otherwise invisible parts of her body while abserving herself in a mirror.

Gallup's empirical findings concerning the phylogenetic distribution of the phenomenon of mirror self-recognition have withstood the test of time (see below, and recent review by Anderson \& Gallup, 1999). However, until recently, the accounts of why these kinds of reactions to seeing the self in a mirror were restricted to chimpanzees, orangutans and humans were sparse (and troubling) - as were detailed theories of the cognitive mechanisms supporting the ability. On the face of it, the fact that this ability was limited to the great apes and humans seemed odd, especially given the difficulty in innagining an ecological context in which evolution would have favored the ability to recognize oneself in a mirror. Rather, self-recognition in mirrors was seen as a byproduct of selection for some other, unspecified abilities (e.g., Humphrey, 1976). Reasoning in this manner, some scholars attempted to link the phenomenon with a higher degree of social intelligence, but this account left unexplained why the ability was not found in the dozens of other highly social primates, but was present in highly solitary orangutans.

The first integrated evolutionary account of why the capacity for selfrecognition in mirrors was restricted to the great apes and humans was provided a decade ago by Povinelli and Cant (1995). Their explanation took a surprising turn, essentially amounting to an account (contra ideas by Gallup, 1982) of how the great 
apes evolved a more explicit representation of their bodies, not their minds. Povinelli and Cant argued that the unique aspects of the self-concept that evolved in the ancestor of the great apes and humans (aspects that are reflected in the mirror test), were largely kinesthenc ones (see Gallup, 1970). They speculated that an explicit representation of some aspect of the kinesthetic component of the self evolved as a result of a peculiar convergence of organismal and ecological factors faced by the common ancestor of the great apes and humans. In particular, they hypothesized that the four-fold difference in the body mass of the great apes (chimpanzees, bonobos, orangutans, and gorillas) as compared to other highly arboreal (tree-dwelling) primates, generated substantial challenges for these animals as they evolved their larger body masses. Povinelli and Cant proposed that this evolutionary increase in body mass rendered the habitat increasingly difficult to traverse, primarily because of the severe deformation of supports (tree limbs) caused by this body mass. Using aspects of the morphology and behavior of modern orangutans as a rough model of the common ancestor, they highlighted how the orangutan's extreme body mass forces it to spend a non-trivial amount of time engaged in locomotor activities that appear to require a great deal of flexibility and planning in the translocation of the body through the arboreal habitat in which it travels. Finally, they speculated that this process of locomotion required the coevolution of a more elaborated representation of the body and its actions to assist in planining and deploying their movements. For purposes of clarity, we shall hereafter refer to this particular body-image' and its attendant systems as "SELF" (Self Evolved for Locomotor Flexibility in large-bodied arboreal primates in highly compliant environments; or 'SELF' for short). Povinelli and Cant further speculated that this self-representational system might have established a quite explicit self concept, one which allows for the behaviors characteristic of mirror selfrecognition, and one which may have served as the point of departure for the more elaborate, psychological aspects of the self-concept so characteristic of humans. In this chapter, we re-visit this model, and clarify and refine certain of its features. We also describe several new predictions that can be derived from the model, along with some preliminary investigations we have conducted to test them.

\footnotetext{
'We use the term 'body image' in conneetion with the conceptual system that evolved in response to selection for a more explicit, conceptual representation of the body and its movements (i.e., the SELF systemy that evolved in the ancestor of the great apes and humans. In subsuming the SELF system under the umbrella of the concept of the "body image", we attempt to follow the standard convention in distinguishing between the concepts of "body image" and "body schena" (see, for example, Gallagher, 1986). Aithough we are not fully comfortable with that distinction as applied here, if one of the central distinctions between 'body image' and 'body schema' is that the former is a conceptual structure whereas the latter is not, then the SELF system, as we envision it, would fall under the rubric of 'body image: One of the only other axes of contrast between 'body image' and 'body schema" in the human literature is the 'onscious versus subconscious' distinction - a dichotomy of little practical use in dealing with non-lhuman primates. A more interesting point of theoretical difficulty is that 'body schema' is typically used when referring to unconscious systems that play a dynamic role in monitoring and governing posture and movement (c.g., Head, 1920).
} 
We begin by reviewing the current evidence concerning species differences in mirror self-recognition. We then provide a brief account of a class of causal models of the phenomenon, and show why self-recognition in mirrors may depend, fundamentally, upon an explicit, kinesthetic representation of the self (SELF) which initially evolved to subserve behavioral solutions to the problems created by the large body mass that evolved in the ancestor of the great apes and humans. Next, we explore the distribution of other abilities in chimpanzees and the great apes which may also either depend upon or be greatly facilitated by the SELF system purported to be uniquely present in these species. In doing so, we describe a preliminary attempt to test the generality of one component of the model by testing whether the SELF system of chimpanzees (and presumably orangutans, as well) expresses itself in situations other than mirror self-recognition (in this case, a tool-using situation).

\section{A SHORT COUIRSE ON MIRROR SELF-RECOGNITION IN PRIMATES AND OTHER ANIMALS}

Three challenges have been raised in response to Gallup's claim that mirror selfrecognition is restricted to the great apes and humans: (1) chimpanzees do not really display the behaviors Gallup reported, (2) chimpanzees do exhibit these behaviors, but so do other non-human primate species (including gorillas and certain species of monkeys), and (3) regardless of whether other primates do or do not exhibit selfrecognition, certain non-primate taxa (such as dolphins) can do so. We briefly examine each of these claims in turn.

\section{Do chimpanzees 'recognize' themselves in mirrors? Yes}

Gallup's (1970) interpretation of the spontaneous self-exploratory behaviors exhibited by chimpanzees (in conjunction with the subjects' responses to the marks tests) was that chimpanzees were capable of using mirrors to explore parts of their bodies that were not previously visible to them. In a widely circulated series of reports, Heyes (1994, 1995, 1998) challenged this (and other aspects) of Gallup"s (1970) interpretation. She hypothesized that the spontaneous self-exploratory behaviors reported by Gallup and others were inherently ambiguous, and that the increase of mark-directed touches from the control to the test period could be explained in other ways. In particular, she argued that chimpanzees normally engage in a significant level of ambient face-touching behavior (e.g. scratching and self-grooming), and therefore speculated that a likely effect of the anesthesia used in the marking procedure would be to depress these baseline levels of face-touching behaviors. She thus argued that the elevation in touches to the marked regions during the test period that Gallup (1970) and others have reported, may have been due to a decline in the effects of the anaesthetic agent across the control and test period. This amounted to a claim that the entire phenomenon of self-recognition in chimpanzees was a methodological artifact.

Povinelli et al. (1997) tested several competing predictions that can be derived from Heyes' model versus the self-recognition model, and in each case 
found clear and unambiguous support for the predictions of the self-recognition model. Indeed, the results were incompatible with every empirical prediction that can be derived from Heyes' model. First, during the mark tests, the chimpanzees selectively touched the marked areas of the face, as compared to the contralateral, unmarked areas (Figure 2). Second, unlike the pattern predicted by a gradual recovery from anesthesia (a gradual increase in touches to the marked areas of the face), there was a clear temporal spike in the chimpanzees" touches to the marked regions coincident with the uncovering of the mirror (see Figure 2). Finally, after spiking, the number of touches to the marks declined across the test (as opposed to increasing and stabilizing at some ambient level, as predicted by Heyes), presumably because the animals discovered their inconsequential nature (see Figure 2).

Although the predictions of Heyes' proposal have been empirically falsified, it should be noted that investigations with larger numbers of chimpanzees of different ages have suggested that certain qualifications of Gallup's (1970) original conclusions are nonetheless warranted. First, there is a much more rapid onset of self-recognition in mirror-naive animals than reported by Gallup (Povinelli et al., 1993). Second, there are developmental dissociations among some types of mirror-mediated, self-directed behaviors and others (Povinelli et al., 1993; Eddy, Gallup, \& Povinelli, 1996). Third, only certain types of mirror-mediated behaviors (e.g. self-exploratory behaviors) are associated with passing the mark test (Povinelli, Rulf, Landau, \& Bierschwale, 1993; Eddy, Gallup, \& Povinelli, 1996). And finally, not all chimpanzees exhibit behaviors diagnostic of self-recognition (Swartz \& Evans, 1991; Povinelli et al., 1993; de Veer, van der Bos, Theall, Gallup, \& Povinelli, 2002). None of these facts, however, suggests that the general phenomenon as reported by Gallup (1970) is unreliable. Indeed, test-retest measures have suggested stability of this trait in many individual animals for up to eight years (de Veer et al., 2002). 
a

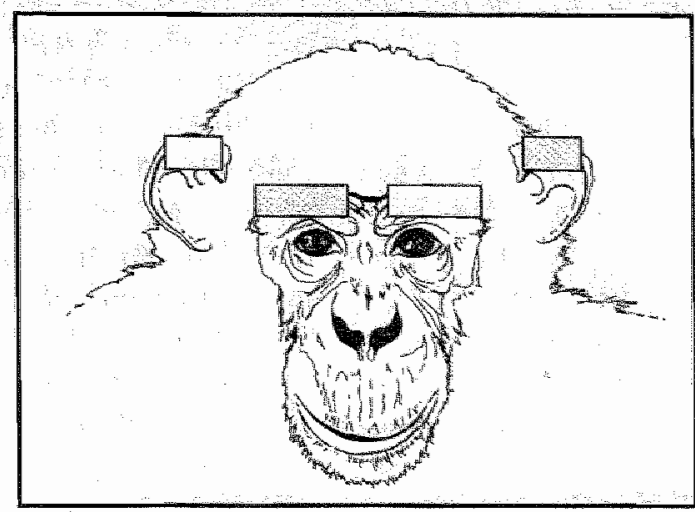

b
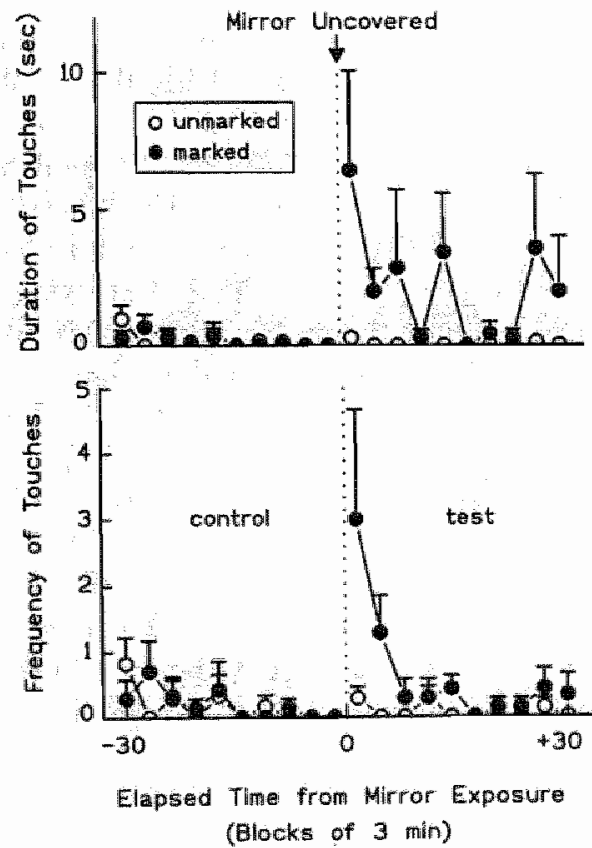

FIGLIRE 2 Method and results of mark test: (a) while under anesthesia, regions of the subject"s face are targeted to be marked with a red dye (shaded areas) and contralateral facial regions (unshaded areas) are targeted as control areas, and upon full recovery (b) subjects is exposed to a covered mirror for 30 minutes, followed immediately by 30 minutes of exposure to a mirror. Note that in (b) touches to the facial regions are selective to the marked areas, coincident with the uncovering of the mirror, and decline after the subject discowers that the marks are inconsequential. 


\section{Is self-recognition absent in lesser apes and monkeys? Apparently so}

Literally hundreds of monkeys, representing a wide array of species, have been tested in the 35 years since Gallup's (1970) original report (see Anderson \& Gallup, 1999). Furthermore, the range of techniques that have been employed to try to "pry" this capacity out of monkeys is impressive (see Anderson \& Gallup, 1999), if for no other reason than that these attempts stand as a constant reminder that the phylogenetic difference in the capacity does not appear to be a trivial one. This is not to say that no one has claimed to have found the capacity in species of nonhuman primates outside the great ape/human group. Perhaps the most celebrated of these was a report by Hauser et al. (1995) who reported to have demonstrated selfrecognition in virtually every cotton-top tamarin that he tested. However, after the original videotapes of this study were made available, and the methodology and interpretation of this study were criticized (e.g., Anderson \& Gallup, 1997), the authors later reported a series of failures to replicate their results (Hauser, Miller, Liu, \& Gupta, 2001).

Naturally, there are two ways of interpreting the inability to detect the capacity for self-recognition in primates outside the great ape/human group. One approach is to try to understand what this difference might mean about the selfrepresentational systems of species (or individual organisms) which do and do not exhibit evidence of self-recognition in mirrors. The other approach is to assume that the differences that have been observed to date do not reflect significant, underlying cognitive differences among species, but instead are superficial artifacts of minor attentional, motivational, and/or perceptual differences. With respect to the latter strategy, numerous methodological explanations have been offered as possible reasons why monkeys do not display mirror-mediated self-exploratory behaviors or pass the mark test. In our estimation, however, none of these possibilities has survived empirical scrutiny (see Anderson \& Gallup, 1999).

Let us briefly examine just two of the most frequently raised methodological concerns surrounding the empirical findings with monkeys. First, it has been widely suggested that because direct eye contact is typically a threatening signal in monkeys, they avoid closely inspecting their mirror images, and hence fail to learn as much about their mirror-image as do chimpanzees and orangutans (see, e.g., Gallup, Wallnau, \& Suarez, 1980). A study by Anderson and Roeder (1989) examined this idea by confronting capuchins monkeys with two mirrors placed at a $60^{\circ}$ angle to each other, thus allowing them to look at themselves without having to make direct eye contact. Despite extended exposure, the capuchins showed no evidence for mirror self-recognition (see also Anderson \& Gallup, 1999, for other attempts to test this hypothesis).

Second, many skeptics have suggested that perhaps monkeys, lesser apes, and even gorillas, are simply not as interested in their bodies as are chimpanzees and orangutans. Thus, perhaps they do not pass the mark test simply because they do not care about the appearance of these marks. Gallup, Wallnau, and Suarez (1980) tested this hypothesis by marking monkeys on their abdomens, as well as on 
their ears and forehead. Upon recovery from anesthesia, the monkeys exhibited an intense interest in touching and inspecting the marks on their abdomens (the ones they could directly see), but not the ones they could only observe through the mirror (the ones on their faces), thus ruling out the idea that they were simply uninterested in such marks. A similar study by Suarez and Gallup (1981) involving four gorillas exposed these animals to mirrors for a total of 80 hours. Despite this extended interaction with mirrors, these gorillas did not attempt to touch the experimentally applied marks on their faces during the mark test, even though they exhibited considerable interest in marks that were placed on their wrists (see also Shillito, Gallup, \& Beck, 1999). This procedure has since emerged as an important motivational control for the mark test in species (or individuals) which do not exhibit evidence of self-recognition (e.g., Povinelli et al., 1993).

\section{Are gorillas a puzzling case? Yes and no}

Gallup's (1970) initial assumption was that the ability for self-recognition might be restricted to the great apes and humans. Extensive testing of gorillas, however, has revealed that these great apes typically do not exhibit the kinds of spontaneous self-exploratory behaviors in front of mirrors displayed by chimpanzees and orangutans, nor have they exhibited evidence of passing properly controlled $^{2}$ mark tests (see Suarez \& Gallup, 1981; Ledbetter \& Basen, 1982; Shillito, Gallup, \& Beck, 1999). On the face of it, this should be puzzling, given the phylogenetic relationships of the great apes and humans (Figure 3).

The orangutan lineage diverged earliest from the last common ancestor of this group, whereas chimpanzees, gorillas, and humans are more closely related. Given the presence of the ability in most species of great apes, but its absence in all other primate species tested thus far (see Figure 3 ), the most parsimonious analysis suggests that the capacity evolved in the last common ancestor of the greatape/human group, and that this capacity was subsequently lost in the gorilla lineage (see Povinelli, 1994). Although the loss of this ability in gorillas may at first seem odd, as we discuss below, this fact turns out to be consistent with the evolutionary model offered by Povinelli and Cant (1995). In other words, the absence of selfrecognition in mirrors in gorillas may be understandable in the context of the clambering hypothesis.

\footnotetext{
2 Three properly controlled tests have not elicited any evidence of self recognition in gorillas (Suatrez \& Gallup, 1981; Ledbetter \& Basen, 1982; Shillito, Gallup, \& Beck, 1999), despite some rather extreme efforts to rule out methodological artifacts (see especially the report by Shillito et al. 1999). Despite this, there have been numerous claims for self-recognition in gorillas (see review by Swartz, Serauw, \& Evans, 1999). However, data supporting the existence of mirror self-recognition in gorillas are derived from tests that have not implemented key control procedures that are necessary in order to draw strong conclusions as to the presence or absence of the capacity (see Gallup, 1994, for a detailled discussion of the logic of various procedures). This is not to rule out the possibility that gorillas, under certain abnormal circumstances) could exhibit evidence of self-recognition (see Povinelli, 1994), but thus far the best evidence suggests that normally-reared, socially housed gorillas do mot.
} 


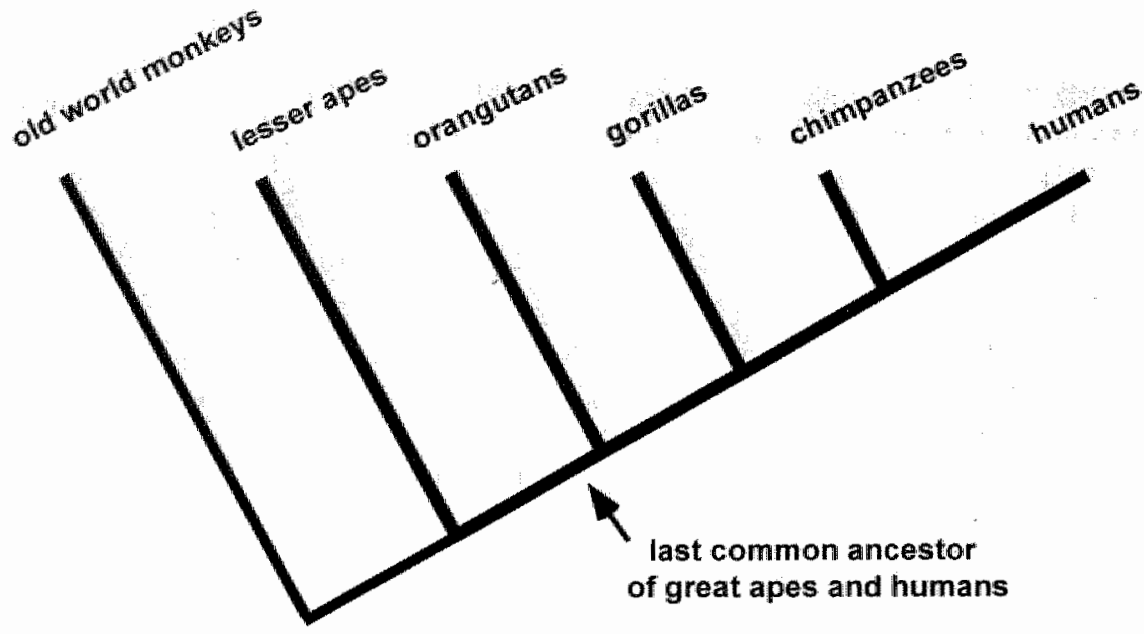

FIGURE 3 Phylogeny of the great apes and humans showing lesser apes (gilbons and siamangs) and old world monkeys as outgroups. The split between the orangutans and the Aftican apes and humans occurred approximately $15-20$ million years ago.

\section{Can dolphins recognize themselves in mirrors? Maybe, maybe not}

Numerous species outside the primate order have been assessed for their reactions to their mirror images (see Gallup, 1968, for a review), but only a handful of them have actually been tested using Gallup's (1970) procedure. Furthermore, until recently, none had been reported to exhibit patterns of behavior suggestive of self-recognition (e.g.r. elephants:" Powinelli, 1989). An exceptron may be dolphins. Reiss and Marino (2001) reported positive evidence of 'self-directed' mirrormediated behavior in two bottle-nosed dolphins, where "self-directed" referred to elevations in the amount of time dolphins spent looking at themselves in mirrors, and the adoption of certain postures while marked. It is important to note, however, that the evidence for self-recognition in dolphins derives from measurements that are not strictly comparable to those used with primates. Two dolphins were repeatedly either marked or sham-marked (i.e., the experimenters merely pretended to mark them) while fully conscious. Dolphins were then reported to swim to a preexisting mirror faster ${ }^{3}$ when they were marked (or sham-marked) and were reported

\footnotetext{
${ }^{3}$ One dolphin was also reported to expose the part of its body that had been marked as the first behavior when it reached the mirror, but this was only true in $6 / 8$ cases, with 2 cases excluded because they were ambiguous. If the 2 ambiguous cases are included as negative instances, this would constitute only $6 / 10$ cases. Because no data conceming how unmarked dolphins typically present their body to a mirror was provided $_{\mathrm{a}}$ it is not olear how to compare these data to a baseline performance. The authors also stress the fact that the same dolphin exhibited no social behavior to the mirrors. However, as the authors thernselves note, the subjecis were thoronghly habituated to the mirrored surfaces in their tanks. Finally,
} 
to spend more time in front of a mirror when marked than when not marked. However, the fact that an organism may increase the amount of time spent looking in a mirror after having been marked does not necessarily indicate self-recognition: For example, over 20 years ago, Gallup and his colleagues showed that rhesus monkeys will exhibit elevated levels of looking at a mirror after having been marked without exhibiting spontaneous selfexploratory behavior or attempting to touch the marks on their face (e.g., Gallup, Wallnau, \& Suarez, 1980). It is as if the monkey in the mirror has become interesting again because of the novelty of its marked face! Nonetheless, despite the reservations we have expressed here, if the findings of Reiss and Marino (2001) are replicated with a design that sumounts the interpretative problems we have expressed, the capacity for self-recognition in dolphins would constitute a remarkable case of convergent evolution, and could point to an alternative evolutionary route for evolving a SELF system.

\section{BODY MASS, HABITAT DEFORMATION, AND THE EVOLUTION OF SELF}

With respect to the phylogenetic distribution of the capacity for self-recognition in mirrors, the evidence just reviewed continues to point in the direction suggested by Gallup over three decades ago: spontaneous, mirror-mediated, self-exploratory patterns of behavior, and positive results using the mark test, appear to be limited to the great apes and humans (with the caveats concerning gorillas and dolphins described above). This means that 15-20 million years ago, some ability evolved in the common ancestor of the great apes and humans that now manifests itself as the capacity for self-recognition in mirrors. But what exactly was this ability, and what was the evolutionary context that produced it? Any attempt to answer this question must begin by providing a clearer specification of what the task is measuring in the first place.

\section{Theory of the mirror self-recogrition task}

As his thinking about the task developed, Gallup (e.g., 1977) increasingly assumed that the patterns of behavior exhibited by chimpanzees in front of mirrors are generated by an inference of the sort, "That's me!" But what dimensions of "me" must necessarily be represented in order to respond to mirrors in this way? Gallup $(1982,1985,1998)$ has emphasized the role of reflecting on one's own mental states. Further, he and others have suggested that the capacity for self-recognition in mirrors in both human infants and chimpanzees may be connected with the emergence of an objective perspective on the self's past and future, by attempting to

the authors also report that no social behavior was observed when mirrors were reintroduced. Unfortunately, no systematic data appears to have been collected to support this point In any event, athough the presence or absence of social behavior to mirrors has besen speculated to be related to selfexploratory bethaviors (e.g., Suarez \& Gallup, 1986), empirical data suggests that this measure does not predict whether chimpanzes exhibit evidence of self-recognition (see Povinelli, Rult, Bierschwale, \& Landau, 1993). 
link the onset of sell-recognition in human toddlers (occurring at around 18-24 months of age) with the emergence of autobiographical memory (see Brooks-Gunn \& Lewis, 1984; Gallup, 1982, 1998, Howe \& Courage, 1993, 1997; Howe, 2004).

But do the behaviors exhibited by chimpanzees and young children in front of mirrors really depend upon representing these psychological and temporal dimensions of the self? Both Mitchell (1993) and Povinelli (1995) have offered alternatives to these views, and have provided detailed analyses of the mirror task which both suggest that mirror-mediated self-exploratory behavior (including passing the mark test) may most directly depend on an explicit representation of one's kinesthetic states - one's body image. Indeed, this general idea seems consistent with Gallup's (1970) original view of the task:

${ }^{" 6}$. . . self-directed and mark-directed behaviors would seem to require the ability to project, as it were, proprioceptive information and kinesthetic feedback onto the reflected visual image so as to coordinate the appropriate visually guided movements via the mirror" (p. 87). However, the exact mechanisms by which infants (and chimpanzees) come to understand the explicit connection between their body and their mirror image has been a matter of some debate, with several theories currently on the table for consideration. In order to gain some insight into the nature of the self-representation that may have evolved in the last common ancestor of the great apes and humans, it is necessary to briefly explore causal theories of the phenomenon of mirror self-recognition in humans.

Mitchell (1993) has argued that the young child (or chimpanzee, for that matter) must first learn that mirrors contingently reflect objects in front of them before they can apply this idea to the case of their own body, and has offered two theories about how this might be accomplished. The existing data would seem to suggest that this premise is incorrect; it is not necessary to understand how mirrors work in order to pass the mark test (discussion by Povinelli, 1995). Research with human infants, for example, has consistently shown that there is no correlation between whether infants pass the mark test and whether they can solve a simple task of turning around to look at a toy that is presented in a mirror (Loveland, 1986; Robinson, Connell, McKenzie, \& Day, 1990). Thus, passing the mark test would not seem to depend upon understanding the pragmatics of mirror use. Further, human infants raised in cultures without mirrors pass the mark test at the same age as infants raised in cultures with mirrors, after only a brief (5-minute) pre-test exposure to their mirror image (Priel \& de Schonen, 1986).

Povinelli's (1995) theory of self-recognition in human infants also emphasizes the role of the kinesthetic dimension of the self, but, unlike Mitchell's theory, specifies that understanding the pragmatics of how mirrors work is not necessary to exhibit patterns of self-exploratory behavior, or to pass a mark test. Instead, his theory pinpoints the existence of an explicit representation of the integrated body image as the critical factor. Once an organism can hold in mind a representation of the current state of its body, it is in the position to begin to form explicit relations among objects of perception (e.g., the image in a mirror) and the 
body image. One such relation that seems critical to exhibit the patterns of behavior that are taken to be criterial for self-recognition in mirrors, is an equivalence relation: That thing (image in the mirror) is equivalent to my body ${ }^{4}$ In analyzing the mirror task, Gallup (1970), Mitchell (1993), and others have emphasized the role of a system that matches (commonly codes) kinesthetic and visual information. While mot denying the role that such common coding must play in the self-recognition task, Povinelli has noted that various forms of imitation are present well before 18 months, some even at birth (see Meltzoff \& Moore, 1999, for a review). Therefore, such systems are not likely to be the limiting factor for the self-recognition task.

The above considerations can be interpreted to suggest that a crucial advance in the development in self-representation in human infants occurs when the kinesthetic aspect of the body is raised to a level of explicitness that it can be held in mind as an object of attention in its own right (Povinelli, 1995). This kind of 'online" representation of the self's bodily actions can be contrasted with an even more advanced understanding of the self as a thing that has repeated instantiations across time - a continuous history.

\section{The present self, extended in time}

Since the early $1990 \mathrm{~s}$, Povinelli and colleagues have been attempting to better understand the kind of on-line, kinesthetic self-representation supporting mirror self-recognition in human infants, by contrasting it with a more temporally extended self-representation that emerges later in development (see Povinelli, 2001, for a review of this research). In one extended series of studies, they explored the role that temporal contingency plays in supporting mirror self-recognition in young children. For example, when the images observed by the young child were not live (as they are in a mirror) but slightly delayed, this strongly affected the children's ability to exhibit evidence of 'recognizing themselves.' In one series of studies, Povinelli, Landau, and Perilloux (1996) exposed 2-, 3- and 4-year-old children to both live and briefly delayed visual images of themselves. In one experiment, each child was videotaped as he or she played a distinctive and novel game with an experimenter. During the game, the experimenter praised the child, and used this as the opportunity to secretly place a large, brightly-colored sticker on top of the child's head. Three minutes later, the children were either shown the video recording of the events that had just happened- including a clear depiction of the experimenter placing the sticker on their head. The results were striking. None of the 2-year-olds, and only $25 \%$ of the 3 -year-olds reached up to search for the sticker. Although it might be tempting to think that the children simply did not notice the

\footnotetext{
"We concede that the presence of the notion of the possession of the body (implied here by the phrase 'my body', as opposed to 'this body'), raises difficult philosophical problems which we do pretend to have solved (see, for example, Martin, 1995). With respect to the mirror task. Davis (1989) has provided a detailed analysis of the minimal kind of self-concept that needs to be ascribed to the organism that passes it. He concludes that the test at least demonstrates the existence of a concept of 'my body' that includes material properties (color, shape, contour, texture, location, movement, etc.).
} 
sticker in the delayed video, this was not the case. Many of them spontaneously commented or laughed when the video depicted the sticker being placed on their head. Furthermore, after the video playback was over, we drew their attention to the sticker on their image in the video, and asked them, "What is that?" The majority of the children responded, "It's a sticker", or something comparable. But significantly, this did not lead them to reach up to their own head to search for it. Even when we asked them if they could get it, they typically looked at the image, shrugged their shoulders and replied, "I can't:" In another experiment, the same basic procedures were used to directly compare how 2-3-year-old children would react to seeing themselves in the standard delayed image, versus a live image. Most of the children in the live condition reached up to remove the sticker, whereas very few children in the delayed condition did so.

We must be careful here. In one sense, 2-3-year-old children can 'recognize themselves' in delayed video or photographs. For example, when asked, "Who is that?", even the youngest of preschoolers we tested replied, "me!" or stated their proper name (see Povinelli et al., 1996, Exp. 2; Povinelli \& Simon, 1998; Povinelli et al., 1999, Exp. 1). However, this recognition seems to occur at the level of their physical features (perhaps most strongly their facial features). For example, when asked, "Where is that sticker?" they frequently made reference to the 'other' child: "It's on her [or his] head." It was as if the children were attempting to say that, "Yes, that looks like me, but it's not me-she's not doing what I'm doing." One 3year-old girl summarized this psychological conflict quite succinctly: "It's Jennifer," she stated - but then hurriedly added, "Why is she wearing my shirt?" Coupled with the results of the mark test, our conclusion from these data are that at any given moment, there are multiples dimensions of the self that might or might not be explicitly represented or 'recognized', and it should not be surprising that at various points in development not all of these aspects of the self may be available to the child.

So when does the child develop a more autobiographical or historical stance toward the self? Our tests reveal that by about 4 years of age a significant majority of children begin to pass our tests of delayed self-recognition, implying that they understand the historical-causal linkage between past and present versions of themselves. Unlike their younger counterparts, 4- to 5-year-olds typically reach up to remove the sticker when they observe the delayed images of themselves. Also, no longer do they refer to 'him' or 'her' when talking about the image, nor do they state their proper name. The transition in performance on our test at 4 years of age is generally consistent with the suggestions by Nelson (1992), Fivush (2001), Welch-Ross (1999), and others, that autobiographical memory may not emerge until 3.5- to 4.5 years of age - considerably later than the 18-24-month mark that others have suggested based in large part on the age at which children begin to recognize themselves in mirrors (e.g., Gallup, 1998; Brooks-Gunn \& Lewis, 1984; Howe \& Courage, 1993, 1997). 
Perhaps the most dramatic confirmation of our interpretation of the delayed self-recognition tests came from a study in which we invited 3-, 4- and 5-year-old children to visit our center twice, with each visit separated by a week (see Povinelli \& Simon, 1998). During the first visit, each child played a novel, distinctive game in a playroom with two experimenters. As in our previous studies, we secretly placed a sticker on their heads. However, at the end of the game, rather than letting them watch the video, we surreptitiously removed the sticker and the child went home. On the second visit, the child played a different game in a different room, during which we once again placed a sticker on his or her head. At this point, the critical manipulation of the study occurred: half of the children in each age group were shown the video recording of what had just happened, whereas the other half were shown the video from one week earlier. The 4- and 5-year-old children understood the difference. In these age groups, the children who saw the briefly delayed video reached up to their heads to search for the sticker, whereas the ones who viewed the week-old tape did not (see Figure 4). In direct contrast, not only did the 3-year-olds reach up less than half of the time, they did so with equal frequency whether they had been shown the 3-minute-old video or the week-old one (see Figure 4). Apparently, not only did the younger children generally not appreciate that the delayed images could be relevant to their current appearance, even the ones who did reach up to their heads did not take into account the critical variable: when the events had occurred in causal relation to the present moment.

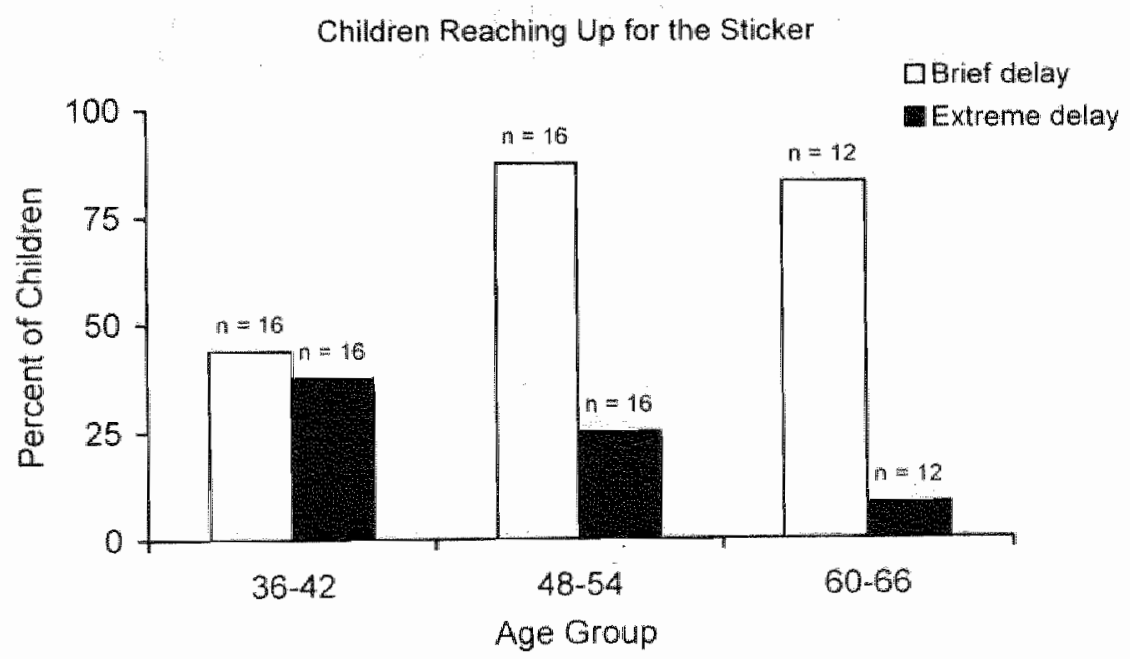

FIGURE 4. Results of a test to determine if 3-, 4- and 5-year-olds can distinguish between the rellevance of videotape showing a sticker having been placed on their heads 7 days earlier (extreme delay) versus 3 minutes earlier (brief delay). As shown here, 4- and 5-year-olds appreciate that only the briefly delayed inages are causally relevant to their current appearance. See text for detalls. 


\section{Summary of the self-system that underwrites the mirror task}

To recap, our theory of the mirror task is that the behaviors which are taken as evidence of "self-recognition" in chimpanzees and very young children are supported by an explicit mental representation of the actions of their bodies (possibly their agency) - not their mental states. We have argued that the mirrormediated behaviors exhibited by chimpanzees and orangutans on the one hand, and young children, on the other, are, at their foundation, derived from an ability to form an explicit equivalence relation between what they see in the mirror and what they directly experience in their own behavior. Every time they act, the mirror (or live video) acts with them. An organism possessing an explicit representation (a concept, as it were) of this kinesthetic experience, ought to be able to conclude that "Everything that"s true of this (my body) is also true of that (the mirror image) - and vice versa." Thus, unlike other species, chimpanzees and orangutans may possess an explicit or integrated enough representation of their bodies to have a concept ('my body') onto which they can map what they see occurring in a mirror. Note that this is not meant to imply that for 18-24-month-old human children the only aspect of the self explicitly represented is the body; rather, it is simply meant to suggest that regardless of whether other aspects of the self (say, the mental aspects) are represented or not, these other representations would not appear to play a pivotal role in eliciting behaviors diagnostic of passing the mirror test. From this perspective, the fact that in human infants the capacity for self-recognition in mirrors is correlated with certain kinds of knowledge of the psychological linkages between self and others, such as those underpinning the self-conscious emotions, synchronic imitation, and empathy (Asendorpf \& Baudonniere, 1993; BischofKöhler, 1988; Lewis, Sullivan, Stanger \& Weiss, 1989; Johnson, 1982) is all the more intriguing, because it suggests that in human development, at least, this leve] of explicitness of the selfs physical aspect is linked in important ways to other kinds of equivalencies between self and other. Whether these equivalences are present in the same fashion in other species that exhibit evidence of self-recognition in mirrors, such as chimpanzees, remains unclear.

\section{Evolutionary origins of SELF: A body too heavy for the trees?}

But then why do humans, chimpanzees and orangutans possess this integrated and explicit bodily self-concept in the first place, while other animals such as monkeys - do not? This question amounts to asking what evolutionary forces sculpted this bodily representation in the last common ancestor of the great ape/human group.

Clearly, any viable theory of the evolution of the kind of body image that we are talking about must begin with the recognition that all organisms with a reasonably developed nervous system must possess dedicated systems for monitoring the effects of external stimuli on the body. In the case of organisms with complex central nervous systems, quite elaborated mechanisms for proprioceptive and kinesthetic feedback are present (see Berlucchi \& Aglioti, 1997). This includes, 
of course, human infants (for discussions of the "ecological" self of human infants, see Butterworth, 1992, 1995). Furthermore, it stands to reason that specific proprioceptive and kinesthetic systems are differentially elaborated in different lineages. However, there would seem to be no a priori reason why these aspects of the self would necessarily need to be represented as objects of knowledge in the central cognitive system in order to function effectively. (Indeed, for what it is worth, such systems are typically taxonomized under the construct of the 'body schema', and therefore placed outside the purview of the system of intentional action; see Gallagher, 1995.)

Thus, to make progress, we must be prepared to accept a distinction between systems which embody proprioceptive and kinesthetic information - that is, systems which are self-specifying for bodily senses - versus systems which represent the body as an object in its own right (akin to the distinction between 'self-perception' and 'self-conception'; see Butterworth, 1992). With this distinction in mind, we need to ask, What factors could have led to the selection for a more robust and integrated representation of the body? or, Why would the ancestors of the great ape/human group, in particular, have needed to evolve a greater capacity to reason about the ongoing effects of the body on the environment during locomotion? As noted earlier, Povinelli and Cant (1995) proposed that this system - what we have for purposes of convenience and clarity labeled the SELF system - evolved to cope with a specific set of organismic and ecological factors that converged in the ancestors of the great apes and humans.

One clue as to why such an explicit representation of the body might have evolved when it did, lies in the large difference in body mass between the great apes and other primates. Consider orangutans, whose body mass and arboreal lifestyle may represent the closest living approximation to the condition of the last common ancestor of the great apes and humans. Povinelli and Cant (1995) noted that these $40 \mathrm{~kg}$ (female) to $80 \mathrm{~kg}$ (male) animals face specific problems in moving from one location to another that are not faced to an appreciable degree by other arboreal primates with smaller body masses. For example, as orangutans attempt to cross from one tree to the next, they not only encounter natural gaps in the canopy, but, due to their extreme body mass acting to deform the limbs of trees, they create gaps, as well. Of course, as any arboreal animal moves outward from the center of a tree, its body mass can, depending on the morphology of the branches, deform the habitat around it, opening gaps between adjacent habitat structures. However, the deformation caused by the very large body mass of orangutans (especially adults) as they attempt to move from one resource patch to the next, creates unusually severe problems because, unlike smaller-bodied arboreal primates, the gaps they create are likely to be larger, and they cannot simply leap across such gaps (in fact, orangutans do not leap at all). Thus, what might be a more or less continuous path for a primate of smaller body mass (punctuated by gaps that they can leap across), will be experienced as a discontinuous path for the orangutan. We also suggest that the combination of the greater deformation caused by greater body mass with variable 
spatial arrangements of branches and lianas (themselves varying in compliance), create greater diversity of gap-crossing challenges for very large animals. Finally, because the relative strength of the habitat is reduced as body mass increases, and each support is less stable as it deforms downward, orangutans must be more selective about the structures they utilize. In summary, we propose that the different nature of discontinuity in the canopy for an arboreal mammal of very large mass, coupled with a general reduction in relative habitat strength and stability, combine to create unique challenges to effective locomotion. Thus an evolutionary increase in one dimension of the animal's body - its mass - has had cascading effects on its locomotion-related activities (Figure 5).

Reflecting on the interacting factors just discussed, Povinelli and Cant ( 1995$)$ hypothesized that the dramatic increase in body mass that occurred during the evolution of the ancestor of the modern great apes and humans, resulted in selection for what we are calling the SELF system, a system that enhances locomotor capacities. The potentially great selective importance of these capacities is apparent from Cant's (1992a) observation that the basic components of reproductive success, such as finding food and mates and avoiding predators, depend directly on the effectiveness with which an animal negotiates its habitat.
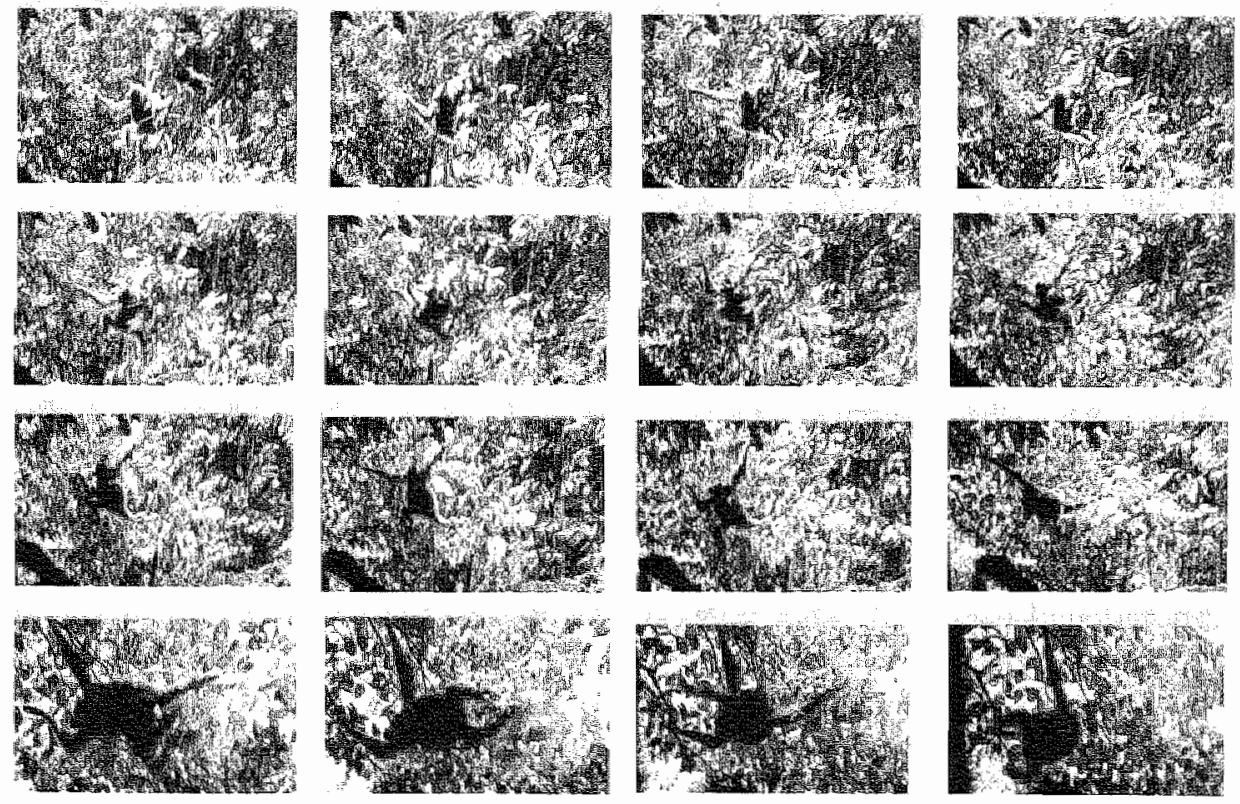

FIGURE 5 An adult female orangutan crossing a gap between two trees by clambering. Note the infant juvenile that she is carrying across the gap. 
Thus, one may reason that as body mass evolved in the great ape/human lineage past some deformation-related limit, and degree of arboreality remained constant, selection would have favored those individuals that could deploy effective new solutions to these problems. The SELF system as we envision it integrates explicit representation of the body, with systems for planning and executing locomotor actions in a highly deformable habitat in which supports used by the animal change their locations not only relative to the animal as it moves, which is true for all moving animals, but in relation to each other due to the loads imposed by the animal itself.

Povinelli and Cant (1995) proposed that one of the principal solutions to the problems described above was the increasing dependence upon a particular mode of locomotion called 'orthograde clambering.' However, it now seems clear that emphasizing a particular mode of locomotion may have diverted attention from the key distinction (admittedly, more difficult to quantify) in the arboreal locomotion of orangutans - a distinction involving a unique process of locomotion through highly compliant habitat structures - and caused us to focus on a behavior (clambering) that is too broadly defined to be useful in this context. Povinelli and Cant proposed that this organismal-ecological interface entailed the co-evolution of a more integrated, explicit representation of the body, along with attendant systems for planning and executing these actions in an arboreal environment: what we have now labeled the SELF system.

It is crucial to note our belief that the problems solved by the SELF system are rarely, if ever, encountered by certain smaller-bodied arboreal primates who live in the same habitat as Sumatran orangutans. Consider long-tailed macaques. Their body mass ( 3.5 to $5.5 \mathrm{~kg}$ ) is generally too small to generate difficult gaps, and thus for them the canopy functions as a more or less continuous substrate. In those cases where natural gaps are encountered, these smaller-bodied primates can simply leap across them - for scaling reasons, an option not available to $40-80 \mathrm{~kg}$ orangutans! Thus, for long-tailed macaques, over $90 \%$ of their daily locomotion can be described by highly stereotyped modes of locomotion (walking, running, leaping, etc.; see Cant, 1988). An intermediate, and therefore interesting case, is the siamang, which may weigh as much as $11 \mathrm{~kg}$. Even this body mass would appear to be large enough to make travel along the top of branches less stable, as the siamang's primary mode of locomotion, brachiation, is suspension bellow branches. However, siamang body mass is apparently not so large as to require the unique process of locomotion practiced by orangutans as they travel through a network of arboreal structures that severely deform under the loads created by their body mass. Siamangs typically cross large gaps in the travel path by leaping on a downward trajectory or dropping (Fleagle, 1976). Furthermore, like long-tailed macaques and other primates, a high proportion of siamang locomotion can be described by stereotyped modes of locomotion. Thus, in contrast to orangutans, other arboreal primates inhabiting the same habitat have apparently not crossed a body mass threshold (the value of which is presently unknown) where deformation of habitat 
structure becomes so severe that a more explicit body-image becomes necessary to keep track of the body's moment-to-moment effect on the surrounding environment.

Although such problem-solving activities of orangutans during locomotion have been qualitatively described elsewhere (Chevalier-Skolnikoff, Galdikas, \& Skolnikoff, 1982, Povinelli \& Cant, 1995; Cant, 1992b), what is needed now is the development of methods that can produce quantifiable estimates of the difference in the complexity of habitat deformation and the orangutan's response to it during bouts of locomotion through highly compliant portions of the arboreal habitat, on the one hand, and locomotion in other contexts where habitat deformation is mild to nonexistent (e.g., locomotion along large, horizontal tree limbs), on the other. A critical component of such research efforts will be the development of reliable methods for describing, and then comparing, the maximum complexity of habitat deformation created by the very large bodied orangutans, to that created by the considerably smaller-bodied arboreal lesser apes and New World monkeys (such as spider monkeys and woolly monkeys) whose body mass (although four times smaller than female orangutans) is still considerable enough to create a non-trivial degree of habitat deformation. Inter-specific comparisons of this kind should allow us to identify the unique "signatures" of habitat deformation generated by very large-bodied apes, as well as their behavioral response to such deformation, with the ultimate aim of more clearly specifying the unique challenges faced by these animals.

\section{Arboreal gap-crossing and life history parameters in comparative perspective}

Focusing just on the four species of extant great apes (chimpanzees, bonobos, orangutans, and gorillas), our model posits a relationship between arboreality, body mass, and body image. (Humans, of course, are a special case that we shall examine in a moment.) Table 1 organizes what is currently known about degree of arboreality, body mass, and mirror self-recognition in the extant great apes. Using the last common ancestor of the great ape/human group as a reference, orangutans appear to have become quite specialized in their behavioral responses to the deformation caused by their body mass while moving through the caanopy, gorillas have moved in the opposite direction, essentially becoming reterrestrialized, with chimpanzees and bonobos somewhere intermediate of these two extremes.

The most intriguing aspect of the data summarized in Table 1 concerns gotillas. Although there are individual differences in mirror self-recognition among chimpanzees and orangutans ${ }^{5}$ (see e.g. Povinelli et al., 1993), at least three well-

\footnotetext{
"Although there would appear to be reliable individual differences in the capacity for mirror selfrecognition among chimpanzees (see Swartz \& Evans, 199 I; Povinelli et al., 1993; deVeer et al., 2002), several researchers have construed the existing data in a highlly questionable manner. For example, at least 3 separate reviews of the self-recognition literature have cited the most definitive population-level study to date, Povinelli et al. (1993), as having produced evidence that only $30 / 92(33 \%)$ of chimpanzees exhibited behavioral evidence of self-recognition $(S R+)$, and/or only $13 / 42(31 \%)$ passed
} 
controlled studies with lowland gorillas have failed to produce even a single animal that has exhibited compelling evidence of self-recognition (see earlier discussion).

Not surprisingly, the gorilla's re-adaptation to a terrestrial way of life appears to have had consequences for certain aspects of their life history. Compared to orangutans, gorillas exhibit an accelerated pattern of development in virtually all areas: they locomote independently at a younger age, they are weaned sooner, and exhibit earlier menarche and age at first birth. Although the data are not excellent, chimpanzees and bonobos again appear to be somewhat intermediate (see Povinelli \& Cant, 1995). Finally, and perhaps more importantly, gorillas appear to develop certain aspects of physical maturity not just absolutely faster, but also when compared to certain aspects of intellectual development (e.g., Redshaw, 1978; Chevalier-Skolnikoff, 1983; Spinozzi \& Natale, 1989; Antinucci, 1990; Parker 1999).

TABLE 1 Mirror Self-Recognition Status, Body Mass, and Complexity of Arboreal Locomotion in the Extant Great Apes.

$\begin{array}{cccc}\text { Species } & \text { Mirror Self-Recognition } & \text { Body Mass Rank } & \text { Complexity of }^{\mathrm{a}} \\ \text { Status } & (\mathrm{kg}, \text { male/female }) & \text { Arboreal Travel }^{\mathrm{b}}\end{array}$

$\begin{array}{lccc}\text { Orangutan } & + & 2(78 / 35) & 1 \\ \text { Bonobo } & + & 4(45 / 33) & 2 \\ \text { Chimpanzee } & + & 3(50 / 41) & 3 \\ \text { Gorilla } & - & 1(169 / 80) & 4 \text { (terrestrial) }\end{array}$

Notes: "Body mass in kg from Fleagle (1999, Table 7.2, body mass among sub-species was averaged). ${ }^{b}$ Species ranks for arboreal travel are deriwed from estimates of time spent in complex positional behavior while crossing from one tree to the next.

One possible conclusion from these data is that as the gorilla lineage was evolving towards a more terrestrial way of life, they no longer needed the SELF system (which evolved to sub-serve behavioral solution to the habitat deformation occurring during arboreal locomotion), and hence were free to "trade off' this

the mark test (see Heyes, 1994; Westergaand \& Hopkins, 1994; Swartz et al., 1999). These numbers were obtained by collapsing across all age groups that Povinelli et al. tested: a grossly flawed procedure given the strong ontogenetic trends that were reported, and highlighted, by the original authors. For example, most of the chimpanzees tested by Povinelli et al. were under 5 years of age, and the data set clearly reveals that the onset of the capacity peaks at around $8-16$ years of age (at which 70-80\% of all animals, based on stringent behavioral criteria, exhibited the capacity). Furthermore, the mark tests specifically targeted an equal sample size of subjects who were classified as $\$ R+$ or $\$ R$ - they were not selected at random as implied by the reviews cited above. To be clear, the analytical technique used by these reviews is analogous to combining data from a large number of 10 -month-old human infants with data from a handful 5-year-old children and adults, in order to obtain an estimate for the percentage of humans that are capable recognizing themselves in mirrors. 
system in favor of more rapid physical maturation. Indeed, data which suggest that gorillas exhibit accelerated physical maturation relative to intellectual development (see above), provide some circumstantial evidence in support of this view (see discussion by Povinelli, 1994). If these data are corroborated, substantial weight would be added to the hypothesis that the basic genetic instructions for those systems are still present in modern gorillas, but are turned off due to shifts in the rate and timing of development (for empirical details which support this possibility, see Povinelli, 1994). However, we should quickly add that it remains unclear what aspects of the SELF system, precisely, might have been traded off during the evolution of gorilla development. It is possible that gorillas possess a body image ${ }^{6}$ indistinguishable from primates outside the great ape/human group. However, it is also possible that they retain a more integrated body image than other primates, but one that differs in substantial enough ways from the ancestral SELF system to preclude the establishment of an equivalence relation between their body and a contingent mirror image of themselves (see above). In other words, only certain developmental pathways relevant to the SELF system may have been turned off during the course of the evolution of the gorilla lineage, and thus these animals may still retain a considerably more developed body image than other primates.

Some researchers have pointed out that the fact that humans are even more re-adapted to a terrestrial way of life than are gorillas could represent a stumbling block for the clambering theory (e.g., Gallup, 1998). As explained above, however, terrestriality is relevant only insofar as it is a marker of selection for more rapid physical maturation relative to intellectual development - that is, to the extent that it suggests that certain later-emerging systems may have been turned off due to changes in the rate and timing of development of other systems. Clearly, this is not true in the human case. In fact, it seems obvious that selective forces that operated during human evolution pushed our species in the opposite direction, developmentally speaking, from gorillas. Rather than accelerating physical development, humans evolved an extended period of physical immaturity and pushed aspects of general intellectual development considerably later, with all key life history parameters delayed relative to the great apes. To provide a concrete example, humans exhibit independent locomotion at a much later stage of cognitive development than do any of the great apes (e.g., Antinucci, 1990). Indeed, if anything, the overall pattern of development in humans suggests an extended period for the elaboration of the self-concept (along many dimensions), not a truncation of this period.

\section{Other "fingerprints" of SELF}

Although the nature of the SELF system is at present only weakly specified, several key aspects of Povinelli and Cant's (1995) theory can now be highlighted.

\footnotetext{
"Given that it is not clear what aspects of the SELF system have been modified, it is unclear whether the term 'body image' or 'body schema' is most appropriate here. See Footnote 1.
} 
First, the hypothesis posits that the great apes and humans possess a body image that is, in some contexts, available as a concept, or object of knowledge in its own right. Whereas many species have proprioceptive and kinesthetic systems which have evolved to regulate complex patterns of movement through space, it may be the case that only the great apes and humans have elevated knowledge of the body as an object of knowledge in itself.

Second, it may be that the SELF is the fundamental platform of the more elaborated dimensions of the self which exist in humans - dimensions which may or may not exist in great apes, and other species. If so, even now it may still be the body, not the mind, which is first and foremost available to the developing human infant, and thus this dimension of the self may retain a kind of phenomenological primacy as other, psychological aspects of the self begin to emerge (see Povinelli, 2001).

Third, if the SELF evolved as an adaptation for coping with the problem that increasing body mass generated for arboreal locomotion in the last common ancestor of the great apes and humans, then its detection millions of years later by Gallup (1970) and his mirror test was curiously serendipitous. After all, if our model has merit, then in attempting to discover whether chimpanzees might be able to recognize themselves in mirrors, Gallup would have inadvertently uncovered a system honed by natural selection millions of years earlier for a completely unrelated function; it just so happened that some of the properties of mirrors happen to engage the SELF system! On this view, we should expect that other abilities may be dramatically facilitated by, or in some cases, even allowed for, by the SELF system. Many authors, for example have discussed the connection between the representation of one's body and imitation (Baldwin, 1901; Merleau-Ponty, 1962; Mitchell, 1993; Meltzoff, 1990). Although many acts of imitation may be possible without the presence of the kind of body-image we are describing, it seems increasingly clear that reproduction of another's actions (broadly construed) can be achieved through multiple routes. Thus, it is possible that the emergence of the SELF system, by adding a level of explicitness to the self, incidentally augmented the tendencies for orangutans and chimpanzees to reproduce the actions of others. Another example may be the case of tool-use. Although many species use, and even fashion tools, in certain species, the presence of the SELF system may facilitate the discovery and deployment of tools as objects separate and explicitly distinct from the thing that deploys them (for example, 'my hand') (see Povinelli, 2000, pp. 328337). In short, the SELF system, which initially evolved as an adaptation to subserve effective locomotion in primates with very large body masses, may express itself (imperfectly) in many contexts, only one of which is mirror selfrecognition.

Importantly, the flip side of this coin may be true as well. It may be that the surprising within-species individual differences in mirror self-recognition among chimpanzees and orangutans (see above) are both real and a reflection of the fact that the fundamental cognitive system that supports mirror self-recognition, evolved 
for other purposes, and therefore only imperfectly map on to the mirror test (or any other test not specifically related to the evolutionary context in which it evolved). In short, individual differences in the robustness of the SELF system that have little or no consequence on the ability to negotiate through highly compliant arboreal habitat may have large differences on tests of mirror self-recognition. This fact may also help explain the previously puzzling findings of declines in mirror self-recognition in adulthood in chimpanzees (see Povinelli et al, 1993; de Veer et al., 2002). After all. what is 'declining' might either be the robustness of the system (not typically used in captivity) or incidental factors which allow for self-recognition, but are not essential to the kinesthetic self needed for effective arboreal locomotion. In addition, this helps to flesh out our earlier explanation of why the absence of behavioral patterns indicative of self-recognition in gorillas may not be so surprising.

It should be emphasized that at present our model is merely a framework pointing to a particular ecological problem (travel through extremely compliant, arboreal habitats by very large-bodied apes) that may have driven a more explicit representation of the body and a suite of motor planning systems designed to subserve effective locomotion. It does not specify (a) the scope of this SELF system, or (b) the extent of the evolutionary 're-description' (sensu Karmiloff-Smith, 1992) that the proprioceptive and/or kinesthetic systems have undergone. Furthermore, it does not specifically indicate which motor planning systems were most affected and/or elaborated by the evolution of the SELF system. In short; at present, the hypothesis fails to specify a causal account of what specific cognitive systems are necessary to support the process of locomotion we have identified as crucial to our model. Because of this present level of ambiguity in the theory, and the theoretical difficulties in reducing that ambiguity, for the time being we advocate a broadbased approach to understanding how the evolutionary forces which sculpted the unique process of arboreal locomotion exhibited by modern orangutans might have affected the representation and use of the body image, broadly construed.

\section{NOVELS TESTS OF THE SELF HYPOTHESIS}

If, as we have been speculating, the mirror test is just one (albeit straight-forward) way of tapping into the SELF system, then a particularly profitable method of testing the clambering hypothesis might be to determine if the great apes differ in as-of-yet undefined ways from other primates in areas related to the use and/or reasoning about the body-image. In what follows, we focus on one approach along these lines that we have pursued. First, we describe how the SELF system may have endowed organisms with a tool-using tendency that, until now has not been widely recognized. Second, we describe a simple experiment that we conducted to determine if chimpanzees, at least, possess this ability.

\section{Tool-use, tool-discovery, and the SELF}

Previously, we had speculated that if chimpanzees and orangutans possess a SELF system, this could explain why they are such expert tool-users: they may 
possess a more explicit representation of their bodies and their actions, and hence they may more clearly consider self-object relations (see Povinelli, 2000, pp. 228337). Tool-use exists in a wide range of animal species (see Beck, 1980, for a broad overview), and we are certainly not suggesting that a SELF system is required to produce such behaviors. Rather, the hypothesis is simply that the more explicitly the distinction between self and object can be represented, the more rapidly tool-use will emerge. The sheer diversity of the tool-kits of chimpanzees in the wild, and the remarkable tool-using and tool-making capacities of chimpanzees and orangutans in captivity, suggest that a more explicit representation of the self's body and actions may dramatically speed up the discovery and use of tools. ${ }^{7}$

The prototypical case of tool use involves the animals using a tool as an extension of the hand in order to rake an out-of-reach object close enough that it can be grasped with the hand. For the purposes of the present research, however, we were interested in the opposite case: where an object of interest is within reach, but the animal does not wish to directly touch it because it is alarming in some way. Would the chimpanzees spontaneously appreciate that a tool could be used to move the alarming object out of the way, or to gather information about it, so that they did not have to touch it directly? It occurred to us that if chimpanzees and orangutans really do possess a more explicit representation of their bodies (by virtue of their possession of the SELF system), then they might exhibit such behavior spontaneously (see Povinelli, 2000, p. 335). After all, if these species really do represent their bodies more explicitly, then as they act upon an object with a tool, they may have at their disposal separately accessible representations of (a) their hand acting on the tool, and (b) the tool acting on the object. ${ }^{8}$

\footnotetext{
${ }^{7}$ A contrast between orangutans and gorillas is of interest here. Orangutans do exhibit tool-making and tool-use in the will (e.g. van Schaik, Fox, \& Sitompul, 1996), but it is certainly not as extensive as chimpanzee tool-making and tool- use. If the definition of tool is expanded to include the remarkable use the deformation of smaller tree trunks to assist in the translocation of their body through space ('tree-swaying'), then the amoumt of orangutan tool-using behavior might easily exceed that of chimpanzees. Indeed, in captivity, orangutars seem to be remarkably pre-adapted to tool-making and tool-use (e.g., Lethmate, 1982). In contrast, gorillas almost never use lools in the wild (even under the broadest definitions available), and although they centainly can and do learn to use tools in captivity, the extent of such behavior does not appear to be as elaborated as iti orangutans and chimpanzees (see Fontanie, Moisson, \& Wicklings, 1995; Boysen et al., 1999). This later claim, however, is largely based on subjective impressions, rather than quantitative data obtained from extensive surveys of zoological collections.

${ }^{\circledR}$ The use of such explicit representations could be contrasted with, for example, studies with monkeys that have shown that when a monkey uses a tool to retrieve distant objects the neuronal schemat that codes for the hand extends to include the space around the distant object (Iriki, Tanaka, $\&$ Iwamura, 1996). Across trials, objects that were represented as being in the extra-personal space, become coded as near objects, if a physical extension of the body (a tool) is used. Although this coding of far objects as near objects in bimodal meurons during tool-tuse has also been found in humans (Farné \& Ladavas, 2000; Berti \& Frassinetti, 2000), we posit an additionall representation in liumans and the great apes which allows for the representation of 'my hand' to mediate these lower-level representations.
} 


\section{An experimental test}

To explore this idea, we tested seven captive adult chimpanzees (Barth \& Povinelli, submitted), all of whom have had an exceptional amount of experience with using tools in a wide variety of tasks (see Povinelli, 2000, for details on their rearing and testing history). The subjects were tested behind a plexiglas partition that contained three openings through which the subjects could freely respond (a procedure with which they were intumately familiar). When the chimpanzees entered, they always found three sticks on the floor of the test unit, always in the same position. Most of the trials were standard tool-using trials, where a piece of food was sitting on the other side of the partition, out of the chimpanzees' immediate reach. On these trials, the chimpanzees easily demonstrated an understanding that they could use one of the sticks to rake in the reward (a hardly surprising feat, given their previous experience; see Povinelli, 2000).

The key findings, however, came from the test trials which were administered at certain intervals between the standard tool-using trials. On these trials, a large box with the open side facing the subject was present on the floor directly in front of the plexiglas partition, and was always positioned the same distance from the subject. A highly desirable food reward (either an apple or a banana) and/or an alarming object were then positioned just inside the box so that they were clearly visible to the chimpanzee and easily within his/her reach. The alarming objects included furry toy animals, rubber snakes, and spiders (the alarming potential of the objects was tested informally on a different group of chimpanzees prior to the beginning of the experiment).

A total of six different kinds of test trials were used (with each animal receiving four trials of each kind). Four of these six conditions consisted of the trials just described: (1) food-only, (2) allarming-object-only, (3) food-besidealarming-object, and (4) food-behind-alarming-object. Two other conditions were also used that were identical to the food-only and alarming-object-only conditions except that the entire box was covered by a cloth so that the subject did not know what was inside unless he or she removed the cloth: (5) covered/food-only and (6) covered/alarming-object-only. As before, the sticks were present, available to the subjects if they chose to use them. Thus, the chimpanzees were free to make contact with the food and/or the object, either by using their hands, using one of the tools as a mediator, or to ignore the situation altogether. The significant aspect of this design was that on all test trials, the food and the alarming objects were always the same distance from, and easily within reach of, the individual chimpanzees.

We reasoned that in the food-beside-alarming-object and food-behindalarming-object conditions, an organism with a SELF system would be highly likely to make the inference that they could use the tool to push the alarming object out of the way and thus avoid having to make direct bodily contact with the object as they reached for the food. Similar logic applied to the two conditions in which the box was covered, and the chimpanzees were therefore ignorant of what was inside: they ought to use the tool to remove the cloth before reaching with their 
hands. Finally, on the alarming-object-only trials we reasoned that in the absence of food, the subjects would be interested in exploring the alarming object, but that they ought to use the tool, not their hands, to do so. All of these conditions could be contrasted with the food-only condition, in which we expected that the subjects would simply reach directly with their hand to take the food. On the other hand, if the subjects simply conceived the tool as an extension of their arm, they would be expected to be quite hesitant to contact the alarming object, even with the tool. Our primary measure was the number of bouts that the subjects used their hands versus the tools across the six conditions.

The two conditions in which the contents of the box were covered turned out to be the most pivotal. Three of our seven apes consistently used one of the provided tools to uncover the concealed box before they reached with their hands in these two conditions (see Figure 6). The remaining four apes either removed the cloth by hand or did not respond at all. The behavior on these "covered" conditions predicted their behavior on the other conditions. The chimpanzees that uncovered the box by using a tool also used the tool consistently on the conditions in which an alarming object was present, but not on the food-only trials. They used the tool to move the object away from the food (or the food away from the object) and they used the tool to explore the object. They only rarely made contact with the object by using their hands, and if they did, only after they first explored it with the tool. Likewise, they only rarely retrieved the food directly without first increasing the distance between food and object by using the tool. In contrast, the four apes that reached to unveil the box with their hands in the covered conditions, also only used the tool randomly with respect to the other conditions, if they used it at all. Importantly, all of the apes directly retrieved the food from the box by using their hands in the food-only condition. This demonstrates that the three apes who used the tool only when the alarming object was present, and when they did not know what was in the box, clearly distinguished between the conditions and did not simply use the tool for idiosyncratic reasons, such as overestimating the distance between themselves and the box. Rather, they used the tool only on those trials during which their hand would have to touch, or come too close to touching, an alarming object.

Although the results are preliminary, they suggest that at least three of our chimpanzees provided reliable evidence of utilizing an explicit representation of their hand when they used the tool as a mediator between their hand and the object. Not all of our apes did so, however. Indeed, our apes could be classified into two groups, those that did use the tool as a mediator and those that did not. This difference cannot be explained by individual differences in tool-using expertise, as all of the apes were proficient tool-users with an exceptional amount of practice and experience on a wide variety of tasks (see Povinelli, 2000). More directly, in the present study, all of the subjects used the tools to retrieve the food reward easily on the standard tool-using trials (see above). So, why did only about half of them use the tool as a mediator? As noted above, perhaps it is because the SELF system we 
are probing evolved in a different context, one that only imperfectly maps itself into the present test situation.
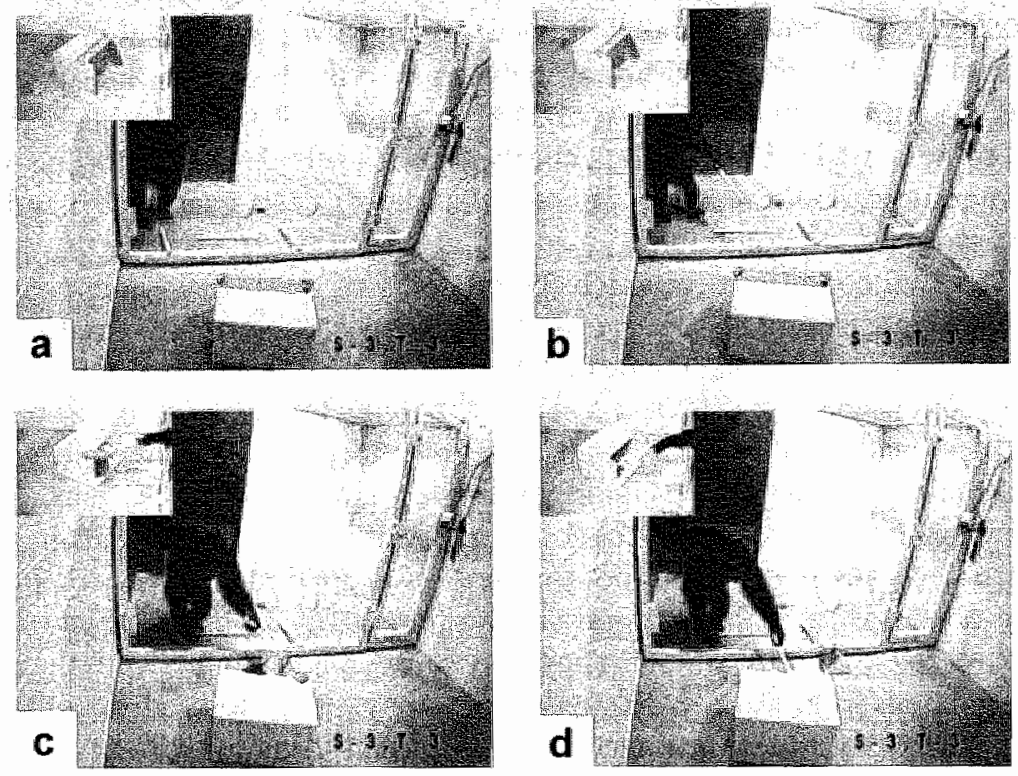

FIGURE 6 Covered box condition: A chimpanzee (a) encounters a covered box and (b-d) spontaneously uses a provided stick to uncover it in order to explore its contents.

Although we have not yet conducted our test with other species - in particular, those that do not exhibit evidence of self-recognition - comparative tests of this sort (especially a direct comparison between great apes and several species of old and new world monkeys) would be of great interest. At present, we offer these data as a possible illustration of how the SELF system we hypothesize to exist in the great apes and humans- a system which has heretofore been explored only through the mirror test- may express itself in other contexts as well.

\section{CONCLUDING REFLECTIONS ON SELF}

In this essay, we have elaborated upon a hypothesis first put forward by Povinelli and Cant (1995) that the evolutionary origins of the self-concept may be found in a unique set of ecological circumstances faced by the ancestors of the great apes and humans - a set of factors that selected for the development of an explicit representation of their body and an understanding of its ongoing effects on the environment. In doing so, we have not sought to downplay the importance of the myriad, implicit kinesthetic and proprioceptive systems that allow organisms to function in the environments they inhabit. Indeed, a wealth of research in developmental psychology, cognitive neuroscience, and motor planning, has 
explored the nature of the body image/schema, elucidating the mechanisms that allow organisms ranging from human infants to bats to fishes to elephants to integrate somatosensory and visual information, and distinguish self from environment.

Nonetheless, it seems likely that a fundamentally new representation of the self - what we have labeled the SELF system - evolved for roughly the reasons outlined almost a decade ago by Povinelli and Cant (1995). But this new, integrated representation of the body may have done more than endow those organisms to exhibit the rather remarkable ability for self-recognition in mirrors. It may have preadapted these species for more elaborated forms of tool-use, new ways of relating to the bodily acts of others, and more flexible use of manual-based signals, just to name a few. And, if we are right, this may just be the tip of the iceberg; it may be that when it comes to cascading effects of the evolution of the SELF, there may exist numerous (and as-of-yet unexplored) species differences between the great apes and humans on the one hand, and most other forms of life on the other. A subtle, but distinct thread may thus run through the actions of humans and the other great apes, a kinematic signature of a SELF we share due to our common ancestry. 


\section{REFERENCES}

Anderson, J. R. \& Roeder, I.-1. (1989). Responses of capuchin monkeys (Cebus apella) to different conditions of mirror-image stimulation. Primates, $30,581-587$.

Anderson, 1 R, \& Gallup G. G. (1997). Self-recognition in Saguinus? A critical essay Animal Behawown, $54,1563-1567$.

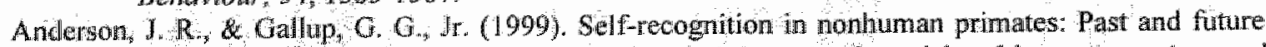
challenges. In M. Haug \& R. E. Whaten (Eds.), Animal models of human emotion and cognition (pp 175\% 194). Washington, DC, US: American Psychological Association.

Antinueci, F. (1990). The comparative study of cognitive ontogeny in four primate species. In S. T. Parker \& K. R: Gibson (Eds), "Language" and intelligence in monkeys and apes: comparative developwental perspectives. New York, NY, US.: Cambridge University Press.

Asendorpf, J B. \& Baudonniere, P.-M. (1993). Self-awareness and other-awareness: Miror selfrecognition and synchronic imitation anong unfamiliar peers. Developmental Psychology, $29,88-95$.

Barth, 1., \& Povinelli, D. J. Tool-mediated object exploration by chimpanzees. Implications for the evolution and acquisition of human tool-use. Mamuscript submitted for publication.

Beck, B. B. (1980), Animal Tool Behowior. New York, NY, US: Garland Publishing, Inc.

Berlucchi, G., \& Aglioti, S. (1997). The body in the brain: neural bases of corporeal awareness. Trends in Neurosciences, 20, 560-564.

Berti, A., Frassinetti, F. (2000). When far becomes near: Remapping of space by tool use. Journal of Cognitive Neurascience, $12,415-420$.

Bischof-Koehler, D. (1988). On the connection between empathy and the ability to recognize oneself in the mirror. [German]. Schweizerische Zeitschrift Fuer Psychologie, 47, 147-159.

Boysen, S. T., Kuhlmeier, V. A., Halliday, P., \& Halliday, Y. M. (1999). Tool use in captive gorillas. In S. T. Parker \& R. W. Mitchell (Eds.), The mentalities of gorillas and orangutans: Comparative perspectives ( $p$ p. 179-187). New York, NY, US: Cambridge University Press.

Brooks-Gunn, J., \& Lewis, M. (1984). The development of early visual self-recognition. Developmental Review, $4,215-239$.

Butterworth, G. (1992). Origins of self-perception in infancy. Psychological Inquiry, 3, 103-111.

Butterworth, G. (1995). An ecological perspective on the origins of self. In J. L. Bermudez \& A. J. Marcel (Eds.), The body and the self (pp. 87-105). Cambridge, MA, US: The MIT Press.

Cant, J. G. (1987). Positional behavior of female Bornean orangutans (Pongo prgmaeus), American Journal of Primatology. 12,71-90.

Cant, J. G. H. (1988), Positional behavior of long-tailed macaques in northen Sumatra. American Journal of Physical Anthropology, 76,29-37.

Cant, J. G. H. (1992a). Positional behavior and body size of arboreal primates: A theoretical framework for field studies and an illustration of its application. American Journal of Physical Arthropology: 88, 273-283.

Cant, J. G. H. (1992b). Positional behavior of arboreal primates and habitat compliance. In B. Thierry, 1. R. Anderson, J. J. Roeder \& N. Herrenschnidt (Eds.). Currem Primatology. Volume 1. Ecology and Evolution (pp. 187-193). Strasbourg: Universite Louns Pasteur.

Cartmil!, M. (1974). Rethinking primate origins. Science, 184, 436-443.

Chevalier-Skolnikot7, 8 . (1983). Sensorimotor development in orang-utans and other primates. Journal of Hunan Evolution. 12,545-561.

Davis, L. H. (1989). Self-consciousness in chimps and pigeons. Philosophical Psychology, 2, 249-259.

de Vea", M., van der Bos, Theall, L. A., Gallup, G.G., Jr, \& Povinelli (2002). An eight-year longitudinal study of self-recognition in chimpanzees. Neuropsycholgia, 1493, 1-6.

Eddy. T. J., Gallup, G. G., Jr., \& Povinelli, D. J. (1996). Age differences in the ability of chimpanzees to distinguish mirror-images of self from video images of others. Joumal of Comparative Psychalogy, 110,38-44.

Farne, A., \& Ladavas, E (2000). Dynamic size-change of hand peripersonal space following tool use. Newroreport, $11,1645-1649$.

Fivush, R. (2001). Owhing experience: Developing subjective perspective in aurobiographical 
narratives, In C. Moore \& K. Lemmon (Bds), The self in time: Developmenol perspectives (pp. 35-52). Mahwah, NJ, US: Lawrence Eulbatim Associates, llnc, Publishers.

Fontaine, B., Moisson, P. Y., \& Wickings, E. Ji. (1995). Observations of spontaneous tool making and tool use in a captive group of western lowland gorillas (Gorilla gorilla gorilla). Folia Primatologica, $65,219-223$.

Gallagher, S. (1986). Body image and body schema: A conceptual clarification. Journol of Mind da Behiovior, 7, $541-554$.

Gallup, G. G., Jr. (1968). Mirror-image stimulation. Psychological Bulletin, 70, 782-793.

Giallup, G. G. (1970). Chimpanzees: Self-recognition. Science, 167, 86-87.

Gallup, G. G. (1977). Self recognition in primates: A comparative approach to the bidirectional properties of consciousness. American Psychologist, 32, 329-338.

Gallup, G. G, Wallnau, L. B. \& Suarez, S. D. (1980). Failure to find self-recognition in mother-infant and infant-infant rhesus monkey pairs. Folia Primatologica, 33, 210-219.

Gallup, G. G. (1982). Self-awareness and the emergence of mind in primates. American Journal of Primatology, 2, 237-248.

Gallup, G. G. (1985). Do minds exist in species other than our own? Neuroscience and Biobehavional Reviews, 9, 631-641.

Gallup, G. G., Jr. (1994). Self-recognition: Research strategies and experimental design. In S. T. Parker \& R. W. Mitchell (Eds.), Self awareness in animals and humans: Developmental perspectives (pp. 35-50). New York, NY, USA. Cambridge Universify Press.

Gallup, G. G., Jr. (1998). Self-awareness and the evolution of social intelligence. Behavioural Processes, 42, 239-247.

Hauser, M. D., Kralik, J., Botto-Mahan, C., Garrett, M., \& Oser, M. (1995). Self-recognition in primates: Phylogeny and the salience of species-typical features. Proceedings of the National Academy of Science, 92, $10811-10814$.

Hauser, M. D., Miller, C. T., Liu, K., G Gubta, R. (2001). Cotton-top tamarins (Saguimus oedipus) fail to show mirror-guided self-exploration. American Journal of Primatology, 53, 131-137.

Head, H. (1920). Studies in Newrology (Vol. 2). London: Oxford University Press.

Heyes, C. M. (1994). Reflections on self-recognition in prinates. Animal Behaviour, 47, 909-919.

Heyes, C. M. (1995). Self-recognition in primates: Further reflections create a hall of mirrors. Animal Behoviour, 50, 1533-1542.

Heyes, C. M. (1998). Theory of mind in nonhuman primates. Behovioral d Brain Sciences, 21, 101134.

Howe, M. L., \& Courage, M. L. (1993). On resolving the enigma of infantile amnesia. Psychologlcal Bulletin, 113, 305-326.

Howe, M. L., \& Courage, M. L. (1997). The emergence and early development of autobiographical menory. Psychological Review, 104, 499-523.

Humphrey, N. K. (1976): The social function of intellect. In P. P. G. Bateson \& R. A. Hinde (Eds.), Growing Points in Ethology (pp. 303-317). Cambridge, UK.: Cambridge University Press.

Hyatt, C. W. \& Hopkins, W. D. (1994). Self-awareness in bonobos and chimpanzees: A comparative perspective. In S.T. Parker \& R. W. Mitchell (Ed.), Selfawareness in Animals and Muman: Developmental Perspectives. New York: Cambridge University Press.

Iriki, A., Tanaka, M., \& Iwamura, Y. (1996). Coding of modified body schema during tool use by macaque postcentral neurons. Neuroveport, 7, 2325-2330.

Johnson, D. B. (1982). Altruistic behavior and the development of the self in infants. Merrill-Palmer Quarterly; 28, 379-388.

Karmiloff-Smith, A. (1992). Beyond modularity: A developmental perspective on cognitive sciente. Cambridge, MA: MIT Press.

Larson, S. K. (1988). Individual differences in physiology, behovior, and temperamen aver the first 1.5 months of life. University of Vllinois, Urbana-Champaign, US.

Ledbetter, D. H., \& Basen, J. A. (1982). Failure to demonstrate self-recognition in gorillas. American Jotanal of Primatology, 2, 307-310.

Lethmate, J. (1982). Tool-using skills of orang-utans. Journal of Human Evolution, 11, 49-64. 
Lewis, M., Sullivan, M. W., Stanger, C., \& Weiss, M. (1989). Self development and self-conscious emotions. Child Development, 60, 146-156.

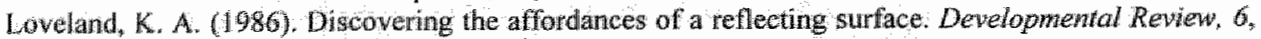
1124.

Martin, M. G. F. (1995). Bodily awareness: A sense of ownership. In J. L. Bermudez, A. J. Marcel \& E. Nami (Eds) The Body and the self. Cambridge, MA, US: The MTT Press.

Meltzoff, A. N. (1990), Foundations for develloping a concept of self: The role of imitation in relating self to other and the value of soclal miroring, social modeling, and self practice in infancy. In D. Cicchetti \& M. Beeghly (Bds.), The self in transition: Infancy to childhood. (pp. 139164). Chicago, IL, US. The University of Chicago Press.

Meltzoff, A. N., Moore, M. K (1999). Persons and representation: Why infant imitation is important for theories of human development. In J. Nadel \& G. Butterworth (Eds.), Imitction in infancy. Canbridge studies in cognitive perceptual development (pp. 9-35). New York, NY, US: Cambridge University Press.

Merleat-Ponty, M. (1962). Phewomewology of Perception. London: Routledge and Kegan Paul.

Mitchell, R. W. (1993). Mental models of mirror-self-recognition: Two theories. New Ideas in Psychology, $11,295-325$.

Nelson, K. (1992). Emergence of autobiographical memory at age 4. Human Development, 35, 172177.

Parker, S. T. (1999). The life history and development of great apes in a comparative perspective. In S. T. Parker \& R. W. Mitchell (Eds.), The mentalities of gorillas and orangutans: Comparative perspectives (pp. 43-69). New York NY, US: Cambridge University Press.

Povinelli, D. J. (1989). Failure to find selfrecognition in Asian elephants (Elephas maximts) in contrast to their use of mirror cues to discover hidden food. Joumal of Comparative Psychology, 103, 122-131.

Povinelli, D. J., Rulf, A. B., Landau, K. R, \& Bierschwale, D. T. (1993). Self-recognition in chimpanzees (Pan troglodytes): Distribution, ontogeny, and patterns of emergence. Joumal of Comparative Psychology, 107, 347-372.

Povinelli, D. J. (1994). How to create self-recognizing gorillas (but don't try it on macaques). In S. T. Parker \& R. W. Mitchell (Eds.), Self awareness in animals and humans: Developmental perspectives (pp. 291-300). New York, NY, USA: Cambridge University Press.

Povinelli, D. J., \& Cant, J.G.H. (1995). Arboreal clambering and the evolution of self-conception. The Qnarterly Review of Biology, 70,393-421.

Povinelli, D. J. (1995). The unduplicated self. In P. Rochat (Ed.), The self in infancy: Theory and reserarch. Advances in psychology. 112 (pp. 161-192). Amsterdam, Netherlands: NorthHolland/Elsevier Science Publishers.

Povinelli, D. J., Landau, K. R., \& Perilloux, II. K. (1996). Self-recognition in young clivildren using delayed versus live feedback: Evidence of a developmental asynchrony. Child Development. $67,1540-155 \%$.

Povinelli, D. J., Gallup, G. O., Jr., Eddy, T. J, \& Bierschwale, D. T. (1997). Chimpanzees recognize themselves in mirrors. Animal Behawions, 53, $1083-1088$.

Povinelli, D. J., \& Simon, B. B. (1998). Young children's understanding of briefly versus extremely delayed images of the self: Emergence of the autobiographical stance: Developmental Psychology, 34, 188-194.

Povinelli, D. I., Landry, A. M., Theall, L. A., Clark, B. R., Castille, C. M. (1999). Development of young children's understanding that the recent past is causally bound to the present. Developmental Psychology, 35, 1426-1439.

Povinelli, D. J. (2000). Folk Physics for Apes. New York, NY, US: Oxford University Press.

Povinelli, D. J. (2001). The self: Elevated in consciousness and extended in time. In C. Moore \& K. Lemmon (Eds.), The self in time: Developmental perspectives (pp. 75-95). Malhwah, $\mathrm{NJ}, \mathrm{US}$ : Lawrence Erlbaum Associates.

Priel, B. \& de Schonen, S. (1986). Self-recognition: A study of a population without mirrors. Journal of Experimental Child Psychology, 41,237-250. 
Redshaw, M. (1978). Cognitive development in human and gorilla infants. Jotarnal of Human Evolution, 7, 133-141.

Reiss, D. \& Marino, L. (2001). Mirror self-recognition in the bottlenose dolphin: A case of cognitive convergence. Proceedings of the Narional Acadeny of Science, $98,5937-5942$.

Robinson, J. A., Connell, S., McKenzie, B. E., \& Day, R. H. (1990). Do infants use their own inages to locate objects reflected in a mirror? Child Development, $61,1558-1568$.

Shillito, D. J., Gallup, G. G., Jr, \& Beck, B. B. (1999). Factors affecting mitror behaviour in westeru Jowland gorillas, Gorilla gorilla. Amimal Behowiour, 57, 999-1004.

Spinozzi, G., \& Natale, F. (1989). Early sensorimotor development in Corilla. In F. Antinucci (Ed.), Cognitive structure and development in nonhuman primates. Hillsdale, NI. US.: Lawrence Erlbaun Associates.

Suarez, S. D., \& Gallup, G. G. (198II). Self-recognition in climpanzees and orangutans, but not gorillas. Joumal of Human Evolution, 10, 175-188.

Suarez, S. D., \& Gallup, G. G., Jr. (1986) Social responding to mirrors in rhesus macaques (Macaca mulatia): Effects of changing mirror location. American Joumal of Primatology, $\| I_{*}, 239$ 244.

Sugardjito, J., \& Van Hoof, J. A. (1986). Age-sex class differences in the positional behaviour of the Sumatran orang-utan (Pongo pygmotews abelii) in the Gunung Leuser National Park, Indonesia. Folia Primatologica, 47, 14-25.

Swartz, K. B., \& Evans, S. (1991). Not all chimpanzees (Pan troglodytes) show self-recognition. Primetes, 32, 483-496.

Swartz, K. B., Sarauw, D., \& Evans, S. (1999). Comparative aspects of mirror self-recognition in great apes. In S. T. Parker \& R. W. Mitchell (Eds.), The mentalities of gorillas and orangutans: Comparative perspectives (pp. 283-411). New York, NY, US: Cambridge University Press.

van Schaik, C. P., Fox, E. A., \& Sitompul, A. F. (1996). Manufacture and use of tools in wild orangutans. Naturwissenschaften, $83,186-188$.

Walrawen, V., Elsacker, L., \& Verheyen, R. (1995). Reactions of a group of pygrny chimpanzees (Pan paniscus) to their mirror-images: Evidence of self-recognition. Primates, 36, 145-150.

Welch-Ross, M. K. (1999). Preschoolers" understanding of mind: Implications for suggestibility. Cognitive Development, 14, 101-131.

Westergaard, G. C., \& Hopkins, W. D. (1994). Theories of mind and self-recognition. American Psychologist, 49,761. 


\section{SUMMARY}

In this dissertation the search and exploration for hidden objects by great apes and young children is discussed. Four experimental studies and one theoretical chapter are presented.

In Part 1, a battery of object displacement tasks was administered to twenty-

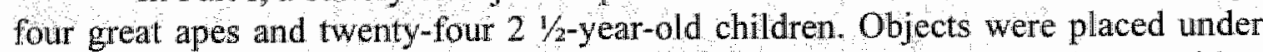
one or two of three cups by visible or invisible displacements and then either remained in the initial location(s) or were moved by rotations or displacements. In these tasks, the subjects had to track the spatial relations of the objects and use that physical information to retrieve them. The battery included six items: (1) Delayed Response, (2) Inhibition Test, (3) A-not-B, (4) Rotations, (5) Transpositions, (6) Object Permanence. We found significant differences between the species and individuals. In general, the apes performed better than the children on the battery. Especially on the Transpositions item, all ape species performed significantly better than the children. Chimpanzees in particular excelled on most items.

In Part II, the ability of great apes and young children to spontaneously use social cues such as gazing, glancing, and pointing as referential communication to locate hidden rewards was investigated. In these studies an experimenter hid an interesting object under one of two cups and then provided a social cue towards the baited cup. In the first study, the subjects were dependent solely on social cues and did not receive any training with non-social cues. Moreover, in contrast to previous studies the cues were not administered with an increasing level of difficulty but in a randomized design. As a group, neither the apes nor the children performed above chance levels at any of the cues. However, in contrast to the apes some children showed results significantly above chance. The limited use of social cues reported in this study is discussed in the context of the variety of experimental designs that have been used to study the use of social cues with object-choice tasks. In the second study, the same task was given to a group of chimpanzees that has been reported to use gaze as a cue to locate hidden food. The same cues as in the first study were given to the target location. However, they were embedded into two different meta-procedures that were modeled after the first study and a previous study in which this group of chimpanzees was reported to use gaze direction as a cue to find the hidden food. Despite the fact that the gaze cue was given in an identical fashion in both procedures, the chimpanzees could use the cue only in one procedure. They were unsuccessful in the same method as the apes in the first study. The difference in the subjects' experience with the meta-procedures was ruled out as an explanation for the difference in the results. These findings imply that (a) chimpanzees can immediately exploit social gaze cues, and that (b) previous conflicting tindings were likely due to the different meta-procedures that were used.

In Part III, the implications of an experimental study on tool-use in which chimpanzees used a tool to explore alarming objects are reported. Chimpanzees were shown a box that either contained food, an alarming object or both. The subjects could touch the contents of the box with their hands or use one of the 
provided tools. Half of the subjects preferred to use a tool if the box contained an alarming object but not when it contained food. The relevance of these findings for the evolution and acquisition of human tool use, including the controlled use of fire by early humans are discussed. In the last chapter the comparative research on selfawareness is reviewed and a theoretical elaboration of its evolutionary origins is offered. The study on tool-use and the theoretical chapter add another aspect of searching and exploring objects, namely by the preferred use of tools as opposed to hands. 


\section{SAMENVATTING}

In dit proefschrift wordt het zoeken en verkennen wan objecten door mensenapen en jonge kinderen besproken. Het bestaat uit vier experimentele studies en een theoretisch hoofdstuk.

In Deel I, werd een batterij van Piagetiaanse, object-verplaatsingstaken aan vierentwintig mensapen en vierentwintig jonge kinderen gepresenteerd. Objecten werden op zichtbare dan wel niet-zichtbare wijze geplaatst onder één of twee van drie bekers en bleven dan op de oorspronkelijke locatie of werden verplaatst door verschuiven of verwisseling van de bekers. In deze taken moesten de participanten de ruimtelijke relaties tussen de voorwerpen bijhouden en die informatie gebruiken om ze terug te vinden. De batterij bestond uit zes onderdelen: (1) Delayed Response, (2) Inhibition Test, (3) A-not-B, (4) Rotations, (5) Transpositions, en (6) Object Permanence. Wij vonden significante verschillen tussen de soorten en tussen individuen. In het algemeen presteerden de mensapen beter dan de kinderen op de testen. Voornamelijk op de Transpostions-taak, presteerden alle soorten mensapen beduidend beter dan de kinderen. Met name de chimpansees blonken uit op de meeste onderdelen. Deze studie is de eerste waarin alle leden van de Pongidae en Hominidae families binnen één studie getest werden.

In Deel II, werd het vermogen van mensapen en jonge kinderen onderzocht om spontaan sociale signalen (cues) zoals kijkrichting of aanwijzen te gebruiken als middel om verborgen beloningen te vinden. In deze studies verborg de experimentator een interessant object onder één van twee bekers en gaf dan met een sociale cue de beker aan waaronder het object verstopt was. In de eerste studie konden de subjecten alleen afgaan op de sociale cues en kregen ze ook geen training met niet-sociale cues. Bovendien werden, in tegenstelling tot wat in eerdere studies was gedaan, de cues niet aangeboden in toenemende mate van moeilijkheid, maar in een willekeurige volgorde. Gemiddeld presteerden zowel mensapen als kinderen niet boven kansniveau voor alle cues. Toch presteerden sommige kinderen wel significant boven kansniveau, in tegenstelling tot de mensapen. Dit slechts in beperkte mate gebruik maken van sociale cues door de subjecten in deze studie werd geplaatst in de context van de verschillen in experimentele methoden zoals die in gelijksoortige onderzoekingen werden toegepast. In de tweede studie werd dezelfde taak bij een groep chimpansees uitgevoerd waarover eerder gerapporteerd was dat zij kijkrichting gebruikten als cue om verborgen voedsel te vinden. Dezelfde cues als in de eerste studie werden gebruikt om de locatie van de beloning aan te geven. Twee verschillende meta-procedures werden hierbij gehanteerd gemodelleerd enerzijds naar onze eigen studie en anderzijds naar de eerdere studie waarin gerapporteerd werd dat deze groep van chimpansees effectief kijkrichting als cue gebruikten. Ondanks het feit dat de cue op identieke wijze werd gepresenteerd in beide procedures, gebruikten de chimpansees de cue slechts in één van beide. $\mathrm{Ze}$ faalden wanneer dezelfde methode als in de eerste studie met mensapen werd toegepast. Uitgesloten werd dat een verschil in ervaring van de chimpansees met de meta-procedures kon gelden als verklaring voor het verschil in 
de resultaten. Deze bevindingen impliceren dat (a) chimpansees onmiddellijk kijkrichting als sociale cue kunnen gebruiken, en dat (b) eerdere afwijkende bevindingen waarschijnlijk het gevolg zijn van verschillen in de toegepaste metaprocedure.

In Deel III, worden de implicaties besproken van een experimentele studie waarin chimpansees een werktuig gebruikten om angstaanjagende voorwerpen te onderzoeken. Chimpansees kregen een doos te zien die ofwel voedsel, een angstaanjagend voorwerp, ofwel beide bevatte. De chimpansees konden de inhoud van de doos met hun handen aanraken of een van de aanwezige werktuigen gebruiken. De helft van de apen verkoos een werktuig indien de doos een angstaanjagend voorwerp bevatte, maar niet wanneer het voedsel bevatte. Besproken wordt de relevantie van deze bevindingen voor de evolutie en ontwikkeling van menselijk werktuiggebruik, inclusief het gecontroleerde gebruik van vuur door de eerste mensen. In het laatste hoofdstuk wordt een overzicht gegeven van het vergelijkende onderzoek naar zelfbewustzijn en de evolutionaire bronnen ervan theoretisch uitgewerkt. Het onderzoek naar werktuiggebruik en het theoretische hoofdstuk voegen een extra aspect toe aan het opzoeken en exploreren van objecten, namelijk de keuze om werktuigen te gebruiken in plaats van handen. 


\section{DANKWOORD}

I. am grateful to Michael Tomasello and Josep Call for the opportunity to work with them and enjoy the unique research conditions at their lab. Henriette Zeidler has been of great help always. Jana Jurkat, Angela Loose, Eva Leermann, and Katharina Haberl supported and helped me with the child studies. All keepers at the Wolfgang Köhler Primate Research Center have been of essential help in the studies with the apes. Mike and Erika Seres have been of a great, although too short, help and company. The many enjoyable moments and laughs with Claudio Tennie, Daniel Hanus, and Hagen Lehmann are unforgotten. I am also grateful to Brain Hare for many passionate discussions. Many, many other people at MPI where of great help and company during this time.

I am also very grateful to my past and present friends and colleagues at the Cognitive Evolution Group. I thank Daniel Povinelli for all his support that I have enjoyed continuously since my first visit as an undergraduate student. I am also very grateful to Jim Reaux, who put a lot of effort into the research reported in Chapter 3. I thank Anthony Rideaux for his care and help with testing the chimpanzees. John Sharp has helped me enormously with about everything.

Wijnand Raaijmakers was niet alleen een excellente co-promoter, die twee keer de lange reis naar Leipzig op zich heeft genomen, om mijn ontwikkeling ter plaatse te bekijken, maar ook een heel prettige vriend met wie ik al vele jaren discussieer en lach. Zijn advies en hulp waren altijd onmisbaar. Hans Stauder heeft me al ontelbare keren waardevol advies gegeven en mij ook in Leipzig bezocht. Verder dank ik Annemie Jeukens die mij in het laatste jaar veel heeft geholpen. Ook gaat mijn dank uit naar Harald Merckelbach, die in het eerste jaar van mijn project me goed advies heeft gegeven.

Weiterhin danke ich ganz herzlich Rainer Goebel, der mich bei den Promotionsvorbereitungen und unserem Workshop sehr unterstützt hat und immer ein offenes Ohr für neue Anregungen hatte. Seine herausragende Professionalität und sein Engagement sind in Kombination mit seiner angenehmen Person eine sehr seltene Erscheinung und haben bei dem Abschließen meiner Promotion und den Vorbereitungen unseres Forschungsworhabens eine wesentliche Rolle gespielt.

Meine Eltern, Gisela und Norbert, haben mich jederzeit voll und ganz unterstützt und meine Begeisterung für das nicht unbedingt auf der Hand liegende Thema Affenforschung, nicht nur respektiert sondern sogar geteilt. Ohne Ihre Unterstützung zu allen Zeitpunkten wäre nichts möglich gewesen. Darum widme ich thnen diese Arbeit von ganzem Herzen. Meine Frau Sanae, hat mich nicht nur jederzeit unterstützt, sondern hat auch durch ihr Wissen und Thre Ratschläge entscheidend zu meiner Arbeit beigetragen. 


\section{CURRICULUM VITAE}

Jochen Barth werd geboren op 11 januari 1974 in Aken, Duitsland. In 1994 behaalde hij zijn Abitur aan het Pius Gymnasium te Aken. Hij studeerde daarna éen jaar Nederlands in Maastricht. In 1995 behaalde hij het Diploma voor Nederlands als Tweede Taal. In september 1995 begon hij met zijn studie Psychologie aan de Universiteit Maastricht die hij in 2001 met het doctoraal diploma afsloot. Hij heeft in 2000 een onderzoeksstage gelopen bij de Cognitive Evolution Group aan de University of Louisiana at Lafayette onder de supervisie van Prof. Daniel Povinelli. In 2001 begon hij als onderzoeksassistent aan het Max Planck Institute for Evolutionary Anthropology in de groep Developmental and Comparative Psychology onder de supervisie van Prof. Michael Tomasello en Dr. Josep Call. In 2002 werd hij AIO aan hetzelfde instiuut waar hij tot medio 2004 vergelijkend onderzoek met kinderen en mensenapen heeft uitgevoerd. Hij is sinds 2002 honorair verbonden aan de groep Neurocognitie, Faculteit der Psychologie, Universiteit Maastricht. Sinds maart 2005 is hij onderzoeksdirecteur bij de Cognitive Evolution Group aan de University of Louisiana at Lafayette. 


\section{MANUSCRIPTS}

Published and in press manuscripts

Barth, J., Reaux, J, E, \& Povinell, D. J. (2005), Chimpanzees' (Pon troglodytes) use of gaze cues in object-hoice tasks: Different methods yield different results. Animal Cagnition, 8, 84 92.

Barth, I., Povinell, D I, \& Cant, J GH (2004). Bodily Origins of SELF. In D. Beike, J. L Lampinen, \& D. A. Behrend (Eds.), The Self and Memory (pp. 11-43). New York Psychology Press.

Povinell, D. $2, \&$ Barth, 1 , (in press) Reinterpreting behavior A human specialization 2 Commentary on Tomasello et al. Understanding and sharing intentions. The origins of cultural cognition. Behowioral and Brain Sciences.

Submilted manuscripts

Barth, J., \& Call, I. Individual and species differences among great apes (Pan troglodytes, Pan paniscus Corilla gorilla, Pongo pygmaeus) and young children (Homo sapiens) in a series of object displacement tasks.

Barth; I., \& Povine II, D I. Tool niediated object-exploration by chimpanzees: Implications for the evolution and acquisition of human tool-use.

Manuscripts in preparation

Barth, J., \& Call, J: Great apes" (Pam troglodytes, Pan paniscus, Gorlla gorilla, Pongo pygmaeus) recall of food location after changes in subject position.

\section{PRESENTATIONS}

\section{Organized meetings}

'Joint Attention: The Prerequisite of Human Culture'. Workshop sponsored by NWO within the programme "Evolution \& Behaviour". Maastricht, The Netherlands. 12-14 November 2004. Coorganized with Rainer Goebel, Wijnand Raaijmakers, and Sanae Okamoto-Barth.

Invited and contributed talks

'Individual and Species Differences in Cognitive Ability: A comparative long-term project with young children and great apes'. Contribured talk at the Annual meeting of the Netherlands Society of Behavioural Biology. Dalfsen, The Netherlands. 26-28 November, 2003.

'Individual and Species Differences among the Great Apes.' Invited talk at the International Symposium COE 2. Exolution of the Apes and the Origin of the Human Beings. Inuyama, Japar. 14-17 November, 2002

\section{Poster presentations}

Barth, J., Reaux, J.E., \& Povinelli, D.J. "Use of social cues to find hidden objects: Different methods yield diffenent results'. 2005 Society for Research in Child Development Biennial meeting. Atlanta, Georgia, 4-7 April 2005.

Barth, J., Call, J., \& Tomasello, M. "Understanding of social cues in an object-choice task. A comparative study with 2.5 -year old children and great apes.' XIV th Biennial International Conference on Infant Studies. Chicago, Illinois, 5-8 May, 2004.

Barth, J., Call, J. 'Assessment of spatial cognition in 2.5-year old children and great apes.' XIVth Biennial International Conference on Infant Studies. Chicago, Illinois, 5-8 May, 2004.

Barth, J., Call, J. 'Great apes' performance in a delayed response task: The effect of movement and mental distraction'. Poster presented at the 8 th Congress of the German Primate Society. Leipzig, Germany. 1-4 October, 2003.

Barth, J. 'Comparative approaches to the evolution and ontogenesis of the mind'. Poster presented at Toward a Science of Consciousness. Tucson IV. Tucson, Arizona. 10-15 April, 2000. 
